\title{
Influences of ecological factors on vocal communication in olive baboons (Papio hamadryas anubis)
}

\author{
Dissertation \\ zur Erlangung des Doktorgrades \\ der Mathematisch-Naturwissenschaftlichen Fakultäten \\ der Georg-August-Universität zu Göttingen
}

Vorgelegt von

Elodie Ey

Aus Mulhouse (Frankreich)

Göttingen, 2008 
D7

Referentin: Prof. Dr. J. Fischer

Korreferent: Prof. Dr. P. M. Kappeler

Tag der mündlichen Prüfung: 28. Oktober 2008 


\section{Contents}

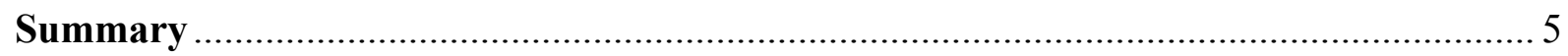

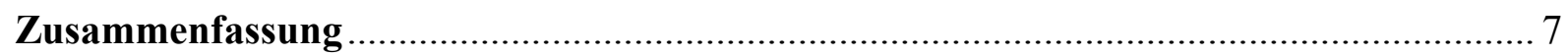

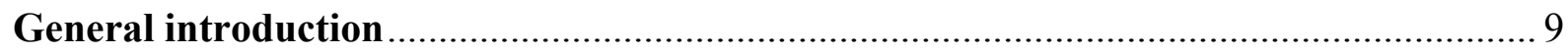

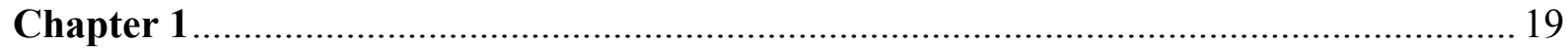

"Keep in touch" - Contact calls in olive baboons

Chapter 2 .

The "acoustic adaptation hypothesis" - A review of the evidence from birds, anurans and mammals

Chapter 3 67

Habitat characterisation - A suggestion for standardised methods and an example of application

Chapter 4

From open to closed habitat - Flexibility in grunt structure and usage in wild female olive baboons

Chapter 5 .

Usage of long-distance communication calls - Limited adjustment to environmental conditions in wild olive baboons

General conclusion

References

Acknowledgements

Appendices

Curriculum vitae 155 


\section{Summary}

In non-human primates, flexibility is largely documented in usage of vocal signals, while it appears more limited in vocal production. The present thesis adopts two perspectives to investigate the degree of flexibility in usage and structure of baboon contact calls -namely grunts, clear barks and loud calls-, which is expected to be highest in usage. First, variability was examined within and between baboon taxa from an evolutionary perspective. Audiorecordings and behavioural data collected in two troops of olive baboons (Papio hamadryas anubis) in Nigeria were compared with data available from other baboon populations and taxa. Second, the degree of intra-individual flexibility was evaluated with a focus on ecological factors. A review of studies in anurans, birds and mammals tested hypotheses concerning environment-related variations in vocal behaviour, and baboon habitat was structurally and acoustically characterised. Then, intra-individual flexibility in response to environmental variations was examined in data on vocal behaviour of the Nigerian olive baboons combined with data from another olive baboon troop in Uganda.

Between olive baboon populations and between baboon taxa, emission rates of grunts, clear barks and loud calls varied importantly, while their contexts of utterance and acoustic structures underwent only minor differences. The review of studies in anurans, birds and mammals revealed that environment-related adjustments in vocal behaviour at a species or population level were generally in line with predictions based on sound propagation features but not as widespread as expected. Since it also underlined the importance of a precise environmental description, closed and open habitats were characterised in the home ranges of the studied olive baboons. Each individual adapted grunt duration to visibility conditions in Nigeria and in Uganda, while grunt rate was adjusted in Uganda only. Contexts of calling and proximity to group members nevertheless constrained this intra-individual flexibility. Regarding vocalisations used over longer distances, baboons tended to avoid high ambient noise level to utter most loud calls. In contrast, emission rate of clear barks seemed to be regulated mainly by other factors than the environment, like predation risk and caller's arousal.

Overall, the evolutionary approach confirms a larger flexibility in usage than in structure of vocalisations between baboon populations and taxa. Surprisingly, intra-individual flexibility in response to environmental variations occurs systematically in grunt production but not in usage of short- and long-distance contact calls. The fine control on vocal production 
in speech might have evolved from this precursory intra-individual plasticity in a common ancestor with humans. 


\section{Zusammenfassung}

Bei nicht-menschlichen Primaten wurde vor allem beim Einsatz von vokalen Signalen eine Flexibilität nachgewiesen, im Gegensatz zur vokalen Produktion, die wesentlich starrer zu sein scheint. In der vorliegenden Arbeit werden zwei Sichtweisen eingenommen, um den Grad der Flexibilität beim Einsatz und der Struktur von Kontaktrufen von Pavianen -Grunzer (grunts), klare Beller (clear barks) und laute Rufe (loud calls)- zu untersuchen, wobei beim Einsatz die höchste Flexibilität erwartet wurde. Zum einen wurde, aus einer evolutiven Perspektive betrachtet, die Variabilität innerhalb und zwischen verschiedenen Paviantaxa untersucht. Hierbei wurden Tonaufnahmen und Verhaltensdaten, die von zwei in Nigeria lebenden Anubispaviangruppen (Papio hamadryas anubis) gesammelt wurden, mit den Daten von anderen Pavianpopulationen und -taxa verglichen. Zum anderen wurde der Grad der intraindividuellen Flexibilität beurteilt mit Schwerpunkt auf ökologische Faktoren. Dazu wurden in einem Review von Studien bei Froschlurchen, Vögeln und Säugern verschiedene Hypothesen über umweltbezogenen Schwankungen des vokalen Verhaltens getestet und das Habitat der untersuchten Paviane bezüglich seiner Struktur und akustischen Eigenschaften charakterisiert. Anschließend wurde die intraindividuelle Flexibilität als Antwort auf Habitatveränderungen untersucht, wobei die Daten vom vokalen Verhalten der nigerianischen Anubispaviane kombiniert wurden mit denen einer in Uganda lebenden Anubispaviangruppe.

Zwischen den Anubispavianpopulationen und zwischen den Paviantaxa schwankte die Rufrate von Grunzern, klaren Bellern und lauten Rufen stark, während die Kontexte in denen die Rufe geäußert wurden und die akustische Struktur der Rufe nur geringe Unterschiede aufwiesen. Der Review von Studien bei Froschlurchen, Vögeln und Säugern zeigte, dass die umweltbezogenen Anpassungen des vokalen Verhaltens auf Art- oder Populationsniveau zum großen Teil mit den auf den Eigenschaften der Schallausbreitung basierenden Vorhersagen übereinstimmten, aber dass diese Anpassungen trotzdem nicht so weit verbreitet waren wie erwartet. Da der Review auch deutlich machte wie wichtig eine detaillierte Beschreibung der Umwelt ist, wurden das offene und geschlossene Habitat des Streifgebietes der untersuchten Anubispaviane genau charakterisiert. Die untersuchten Paviane passten ihre Grunzdauer an die Sichtbarkeitsbedingungen des Habitats an, sowohl in Nigeria als auch in Uganda. Die Grunzrate wurde dagegen nur von den Pavianen aus Uganda an die Sichtbedingungen angepasst. Allerdings beschränkten der Kontext in dem gerufen wurde und die Nähe zu Gruppenmitgliedern diese intraindividuelle Flexibilität. Bei Vokalisationen, die über lange 
Distanzen verwendet werden, tendierten die Paviane dazu, laute Rufe bei einem niedrigen Geräuschpegel der Umgebung zu äußern. Im Gegensatz dazu schien die Rufrate von klaren Bellern hauptsächlich von anderen Faktoren reguliert zu werden, wie einer erhöhten Prädationsgefahr oder der Erregung des rufenden Tieres.

Insgesamt bestätigt die evolutive Herangehensweise, dass es eine größere Flexibilität beim Einsatz als bei der Struktur der Vokalisationen zwischen den Pavianpopulationen und taxa gibt. Überraschenderweise trat die intraindividuelle Flexibilität als Antwort auf Habitatveränderungen systematisch bei der Produktion von Grunzern auf, aber nicht beim Einsatz von Rufen über kurze oder lange Distanzen. Diese intraindividuelle Plastizität könnte die Vorstufe eines gemeinsamen Vorfahren mit dem Menschen widerspiegeln, aus der sich die Fähigkeit der genauen Kontrolle der vokalen Produktion entwickelt hat, welche notwendig war für die Evolution der Sprache. 


\section{General introduction}

Vocal communication has evolved in various taxa, for instance in anurans, birds and mammals, and plays a crucial role in reproductive and survival strategies. Some analogies occur across taxa. For instance, vocal communication systems of birds, pinnipeds, cetaceans and humans share some characteristics despite the large phylogenetic distances which separate these groups. Flexibility in usage and structure of vocal signals within individuals is one of these. Indeed, in some birds (e.g., songbirds, parrots, hummingbirds; Marler 1970; Todt 2004), pinnipeds (e.g., harbour seal [Phoca vitulina]: Ralls et al. 1985; walrus [Odobenus rosmarus divergens]: Schusterman \& Reichmuth 2008), cetaceans (e.g., bottlenose dolphins [Tursiops truncatus]: Janik 2000), and humans (Homo sapiens; e.g., Hauser 1996; de Boer 2005), usage of vocal signals can be conditioned without much trouble, vocal production is largely determined by learning and vocal imitation is common (see Janik \& Slater 1997 for a review in mammals). Non-human primates (hereafter primates) also show some intra-individual flexibility in usage of vocal signals. However, in this group, the degree of intra-individual plasticity in vocal production is surprisingly limited (reviewed in Seyfarth \& Cheney 1997; Janik \& Slater 1997, 2000; see hereafter) and the few experiments of vocal imitation were not a plain success (chimpanzee [Pan troglodytes]: Hayes \& Nissen 1971). This difference in the degree of intra-individual flexibility in vocal production between humans and primates is intriguing, since humans are phylogenetically much closer to primates than they are to birds, pinnipeds or cetaceans. This observation raises questions about the abilities of primates to adjust their vocal behaviour to variations in different factors such as social organisation and environment and about which aspects of vocal behaviour are adjusted (if adjustment occurs). A first approach to clarify these questions lies at a group or individual level. Indeed, flexibility as a response to social or environmental variations can be examined within groups or individuals ranging in different social surroundings or environmental conditions. A second approach implies an evolutionary perspective in which social- or environmental-related variations are examined between species or between populations or groups. This more general approach necessitates some control on the phylogenetic relatedness between species, populations or groups to avoid confounding its effect with other factors. The present thesis privileges the first approach, while also providing data which should allow discussion from an evolutionary perspective. 


\section{$\underline{\text { Intra-individual flexibility in vocal communication in primates }}$}

In primates, usage of vocal signals is known to be flexible within individuals. Experience plays an important role in understanding call meaning and contexts of utterance (reviewed in Seyfarth \& Cheney 1997, Hammerschmidt \& Fischer 2008). For instance, young vervet monkeys (previously Cercopithecus aethiops, now classified as Chlorocebus pygerythrus) gradually become more specific in their usage of different alarm call types in response to different predator types (reviewed in Cheney \& Seyfarth 1990). They also learn appropriate reactions to the different alarm call types by copying the behaviour of adult individuals (Seyfarth \& Cheney 1986). Several studies also reported a successful conditioning of usage of vocal signals in several primate species: individuals learn to utter calls in response to particular visual cues (reviewed in Pierce 1985). Some primates even adjust the timing of their calls to utter them mostly in predictable time intervals with low background noise level (cotton-top tamarins [Saguinus oedipus]: Egnor et al. 2007).

In contrast, call production (i.e., the different call types in the vocal repertoire and the acoustic structure of a call type) is considered to be largely innate (Jürgens \& Ploog 1981; Jürgens 2002; reviewed in Seyfarth \& Cheney 1997). For instance, squirrel monkeys (Saimiri sciureus) do not need to listen to conspecific vocalisations or to have auditory feedback from their own calls to develop their normal vocal repertoire (Winter et al. 1973; Hammerschmidt et al. 2001). Despite this apparent strong neural constraint in call production, a limited intraindividual flexibility has nevertheless been reported (reviewed in Janik \& Slater 1997, Hammerschmidt \& Fischer 2008). Structural variations occur within a genetically fixed frame in response to various factors (see hereafter). This reveals some plasticity in the neural circuitry involved in vocal production.

Intra-individual flexibility in vocal production has been related to several factors (ontogenetic changes due to maturation of vocal production organs [e.g., Hammerschmidt et al. 2000, 2001; Ey et al. 2007b] are not considered here). First, conditioning experiments have shown that some primate species can learn to lengthen their vocalisations (rhesus macaques: Sutton et al. 1973). Second, the distance between a caller and a recipient can explain variations in acoustic structure of some calls (e.g., Masataka \& Symmes 1986; Oda 1996; Sugiura 2007). For instance, isolation calls of squirrel monkeys (Masataka \& Symmes 1986) and coo calls of Japanese macaques (Macaca fuscata: Sugiura 2007) are longer when callers are far away from other group members than when they are closer to one another. Third, call structure might vary according to the caller's dominance rank (chacma baboon [Papio hamadryas ursinus] loud calls: Fischer et al. 2004). Fourth, the surrounding by other 
conspecific groups (pygmy marmoset [Cebuella pygmaea] trills: Elowson \& Snowdon 1994) and group composition and social relationships between animals (Campbell's monkey [Cercopithecus c. campbelli] harmonic calls: Lemasson et al. 2003, Lemasson \& Hausberger 2004) also induce variations in call structure. Fifth, context-related variations also occur in various call types and species (e.g., Barbary macaque [Macaca sylvanus] disturbance calls: Fischer et al. 1995; chacma baboon grunts: Owren et al. 1997, Meise 2008; Diana monkey [Cercopithecus diana] alarm calls: Zuberbühler et al. 1997). Sixth, the level of arousal can also be a factor leading to intra-individual variability (chacma baboon grunts: Rendall 2003, Meise 2008; probably in Japanese macaque coo calls: Koda 2004). For instance, chacma baboons utter grunts of longer duration, with higher fundamental frequency, steeper contours of fundamental frequency, increased "breathiness" or "hoarseness" and lower second formant frequency when they are highly aroused, in comparison to when their arousal level is low (Rendall 2003; see also Meise 2008). And last but not least, an increase in ambient noise level might also explain an increase in call amplitude (as in the Lombard effect; Lombard 1911) and / or call duration (macaque [Macaca fascicularis, M. nemestrina] coo calls: Sinnott et al. 1975; common marmoset [Callithrix jacchus] twitter calls: Brumm et al. 2004; cottontop tamarin combination long calls: Egnor \& Hauser 2006). To summarise, various social (distance to conspecifics, dominance rank, neighbour's identity, social relationships), contextual (conditioning, situation of calling), internal (arousal), and ecological (ambient noise level) factors seem to trigger some intra-individual variability in acoustic structure of vocal signals.

\section{Limited investigations of the influences of ecological factors}

According to the short above-cited inventory, investigations of influences of ecological factors at the intra-individual level remain restricted to ambient noise level in primates. Other environmental features such as vegetation density and transmission properties (i.e., absorption, reverberation) have not been examined. These characteristics specific to each environment lead to specific attenuation and distortion of propagating sounds (reviewed in Delany 1977). Individuals are therefore expected to adjust usage and / or acoustic structure of their vocalisations to these conditions to optimise transmission ("acoustic adaptation hypothesis": Morton 1975 [this hypothesis originally concerns acoustic structure but it is extended to usage in this thesis]; summarised in Brenowitz 1986, Endler 1992). Such adaptations should occur when signals are intended to travel undistorted over relatively long 
distances. However, in some cases, vocal behaviour is on purpose not adjusted to maximise signal detection and propagation distance. For instance, calls might be designed to degrade at a determined rate to allow recipients to estimate the distance between the caller and themselves ("ranging" process: Morton 1986; reviewed in Naguib \& Wiley 2001; Nemeth et al. 2006). In other cases, animals might adjust their signalling behaviour in order to reduce eavesdropping by predators. They might then avoid exposed places for calling or use calls which carry only over short distances (e.g., Tuttle \& Ryan 1982; Endler 1992; Forrest 1994). Such particular situations should be taken into account when examining environmental influences on vocal behaviour. In the present thesis, it will be generally assumed that the vocalisations under study in chapters 4 and 5 are intended to travel with minimal degradation relatively far since there is no expectation of ranging process and predation risk is very low at the study sites.

So far, most work on the influences of environmental conditions on vocal communication is based on birds. The few similar studies conducted in primates investigate this question mainly with an evolutionary perspective. They compare species or populations living in different environments (reviewed in Chapter 2). Only one study in chacma baboons documents variations in call rate according to the habitat type at an intra-individual level (Rendall et al. 2000). Studies documenting an intra-individual plasticity in acoustic structure are only testing the influence of ambient noise level (see above). It is therefore not known whether primates also show some intra-individual flexibility to adjust their vocal production and usage of their calls- to their immediate environment characterised by vegetation density and transmission features. Unravelling this question in a carefully selected species will be the major aim of this thesis.

\section{Baboons as a model to study flexibility in vocal communication}

Investigating intra-individual plasticity as a response to ecological conditions in primates requires finding a model with individuals ranging in different environments. Besides, it would be interesting to complement this approach by an evolutionary perspective and investigate variability in vocal behaviour between populations and / or taxa. This should allow to examine how selective pressures such as environment but also social organisation shape the vocal communication systems within and between taxa. A primate model providing some control on phylogenetic relatedness would then be particularly suitable to rule out the genetic influence and isolate effects of environmental or social factors. In addition, an ideal 
model should permit combination of the social and environmental effects. This should allow to understand the interplay of these factors by studying groups with different social organisations in various environmental conditions. These two factors are tightly related, with environmental conditions shaping social organisation (Wind 1992; Henzi \& Barrett 2003).

Baboons (genus Papio) lend themselves for such an integrative study since the three following characteristics indicate that they fulfil the criteria cited just above. First, the five typically distinguished morphotypes of baboons are widely distributed across sub-Saharan Africa (de Vore \& Hall 1965; Kingdon 1997; Sarmiento 1997; Zinner et al. 2008). Chacma baboons live in the south of Africa; yellow baboons (Papio hamadryas cynocephalus) range north of them. East and Central Africa and a large part of West Africa are occupied by olive baboons ( $P$. h. anubis). Guinea baboons ( $P . h$. papio) can be found at the extreme west, and hamadryas baboons (P. h. hamadryas) range near the Arabic peninsula (Kingdon 1997; Zinner et al. 2008). Recent investigations shed some light on the phylogenetic relatedness between the different taxa and, to some extent, between populations over Africa (Zinner et al. 2008). Second, because of their broad geographic distribution, baboons range in different habitat types, with variable ecological conditions. Hamadryas baboons range mostly in semidesert areas (Kummer 1968), chacma baboons in easily flooded grassland interspersed with patches of woodland (e.g., Cheney et al. 2004; Fischer et al. 2001a), woodlands (e.g., Ron et al. 1996), mountainous areas (Hall 1963) and up to desert edges (e.g., Davies \& Cowlishaw 1996). Guinea baboons live in savannah woodlands and bushy areas (Dunbar \& Nathan 1972) as well as in woodland savannah with stripes of gallery forest (Byrne 1981). Olive baboons range in very diverse environments, such as open grassland (de Vore \& Hall 1965; Harding 1976), gallery forest and grassland (Rowell 1966), savannah (Strum 1987), grassland clearings and moist semi-deciduous tropical forest (Rahn 2008), and -at the site thoroughly studied in the present thesis- in woodland savannah, lowland rainforest and riverine forest. Third, diversity in social organisation in baboon taxa (Kingdon 1997; summarised in Maestripieri et al. 2007) is believed to have emerged from the interplay of various ecological factors, such as climatic conditions, resource availability and predation risk (reviewed in Henzi \& Barrett 2003). Hamadryas baboons live in one-male-multi-female groups (harems), which gather into much larger troops (Kummer 1968). Chacma (e.g., Hall 1963), yellow (e.g., Semple et al. 2002) and olive baboons (de Vore \& Hall 1965; Rowell 1966) live in multimale-multi-female groups. Guinea baboons represent the least investigated baboon taxon. They seem to live in complex fission-fusion multi-male-multi-female groups (Dunbar \& Nathan 1972; Byrne 1981). The core of Guinea baboon societies may consist of one-male 
units (Maestripieri et al. 2007), reflecting a harem structure as in hamadryas baboons. However, recent observations of a group of wild Guinea baboons in Senegal suggest otherwise (J. Fischer, D. Zinner, pers. comm.). Social systems can also be intermediate between different forms in hybridisation zones (e.g., Bergman \& Beehner 2004). As stated above, all these aspects make the genus Papio a suitable model to investigate variability in vocal communication from an evolutionary perspective.

\section{Current knowledge on baboon vocal communication}

Long-term behavioural data from several baboon populations such as Amboseli (e.g., Altmann \& Altmann 1970; Altmann 1980), Gilgil and Chololo (e.g., Harding 1976; Strum 1987), Gombe (Ransom 1981), de Hoop (e.g., Hill et al. 2003) and Moremi (e.g., Cheney et al. 2004) are now available and a number of other populations are accessible for further investigations. Wild baboons are indeed subjects of an impressive amount of studies on diverse subjects, such as phylogenetic relatedness (e.g., Newmann et al. 2004; Zinner et al. 2008), sexual behaviour and life history (e.g., Hill et al. 2000; Higham et al. 2007, 2008), social systems (e.g., Kummer 1968; Bergman \& Beehner 2004), social relationships (e.g., Palombit et al. 1997; Palombit et al. 2001; Lemasson et al. 2008), foraging behaviour (e.g., Harding 1976; Hill \& Dunbar 2002), feeding ecology (e.g., Kunz \& Linsenmair 2008a, 2008b), group coordination (e.g., Stueckle \& Zinner 2008) and vocal communication (see hereafter).

Among other primates, the contribution of baboons to knowledge on primate vocal communication is substantial. As baboons are mostly terrestrial and many populations range in open environments, it is easier to observe them and record their vocalisations in comparison to many other primate species, especially arboreal ones (Henzi \& Barrett 2003). However, most of the current knowledge on baboon vocal communication is based on chacma baboons (reviewed in Cheney \& Seyfarth 2007). In this taxon, both usage (i.e., contexts in which calls are given, rate of calling, age and sex classes of callers [e.g., Cheney et al. 1995, 1996; Palombit et al. 1999; Rendall et al. 1999; Fischer et al. 2001b]), and structure (i.e., acoustic features and their variations with contexts and caller's characteristics [e.g., O’Connell \& Cowlishaw 1994; Owren et al. 1997; Fischer et al. 2001a, 2002, 2004; Rendall et al. 2004]) of vocal signals have been investigated. In contrast, vocal communication in other baboon taxa is poorly investigated. For instance, the last comprehensive studies describing vocal behaviour in olive baboons were published more than twenty years ago (Hall 
\& de Vore 1965; Rowell 1966; Ransom 1981). Otherwise, only a few abstracts on usage and function of grunts and copulation calls (Gilmore 1978, 1979, 1983a, 1983b) and a recent study on friendship between males and lactating females, using playbacks of screams to elicit support (Lemasson et al. 2008), have been published. In Guinea baboons, an investigation on usage of clear barks (Byrne 1981) has also been published more than twenty years ago, and, since then, only the function of copulation calls was studied (Maestripieri et al. 2005). Vocalisations of baboon taxa other than chacma baboons were also rarely investigated with modern techniques of acoustic analyses (but see Semple et al. 2002 for a study on copulation calls in yellow baboons, and Pfefferle \& Fischer 2006 for a study on grunts in hamadryas baboons). In sum, little is currently known about the variability in vocal behaviour (or lack thereof) which might exist among baboon taxa and populations. The present thesis aims to contribute to its documentation.

\section{$\underline{\text { Vocalisations and species under study }}$}

A long-term project -which the present thesis belongs to- aims to unravel the interplay of factors such as phylogenetic relatedness, social organisation and environment in the evolution of vocal communication in the genus Papio. Variability in usage and structure of distinctive vocal signals occurring in all baboon taxa should then be documented. For such a project, contact calls (i.e., grunts, clear barks and loud calls) are particularly relevant to study, since equivalent calls are found in all baboon taxa. Grunts are used in within-group communication over short distances. Clear barks and loud calls are used in communication over long distances, within groups for the former and presumably within and between groups for the later (see Chapter 1). Grunts and clear calls clearly own characteristics of contact calls (reviewed in Chapter 1), while the categorisation of loud calls as contact vocalisations is more disputable. Indeed, this call type is not restricted to situations clearly involving contact maintenance between groups or individuals but can also be uttered in other situations, for instance after aggressive interactions involving a female of the troop (see Chapter 1). Loud calls might nevertheless sometimes serve to monitor other groups, and they will be considered as contact calls in the present thesis for this reason.

Olive baboons were chosen as a study species. They range in the most diverse habitat types among all baboon taxa, from open savannah to riverine forest, with all intermediates possible (see above). It is even possible to find troops of this taxon ranging in both closed habitats like forest and open habitats like grassland. This was the case in two troops in 
Gashaka-Gumti National Park, Nigeria, and in one troop in Budongo Forest Reserve, Uganda. Such conditions are ideal to examine whether olive baboons individually adapt their vocal behaviour to the habitat in which they range, since the same individuals can be recorded under very different environmental conditions. I collected most data for this thesis in GashakaGumti National Park, and then I combined them with data collected by Charlotte Rahn in Budongo Forest Reserve.

Olive baboons are large, heavily-built primates living in multi-male-multi-female groups. Their diet is diversified, mostly herbivorous and frugivorous, and complemented whenever possible by animal proteins such as ants, grasshoppers, crabs or even small vertebrates (de Vore \& Hall 1965; Rowell 1966; Ransom 1981; Kingdon 1997; pers. obs.). They have been largely studied for other aspects than their vocal behaviour, for instance life history and reproductive strategies (Higham 2006; Higham et al. 2008), seed dispersal (Kunz \& Linsemair 2008a, 2008b), behavioural ecology (Warren 2003), social relationships (Castles et al. 1999; Lemasson et al. 2008) and cognition (Vick \& Anderson 2003). Nevertheless, up to now, only a general description of their vocal repertoire is available (see above). Other aspects of their vocal behaviour, like call rates and acoustic features of their contact calls, were not investigated further. The present thesis aims to fill this gap.

\section{Aims of the thesis}

To sum up, this thesis has two major aims:

- to begin investigations on variability in vocal communication between olive baboon populations and between baboon taxa,

- to evaluate the degree of intra-individual flexibility in response to variations in environmental conditions in olive baboon vocal behaviour.

Generally, usage of contact calls is predicted to be more flexible than their acoustic structure (see above). Phylogenetic constrain on acoustic structure is expected to allow only minor variations (if any) between baboon populations and taxa, while emission rates and contexts of calling should vary more importantly between populations and taxa according to social or environmental conditions. Vocal behaviour should also be adapted to optimise propagation distance of signals. Environment-related adjustments are expected to be important in usage of contact calls, while their acoustic structure is supposed to be genetically fixed and therefore show only a limited flexibility (if at all) in response to variations in 
environmental conditions. These general hypotheses on baboon vocal behaviour will be tested across the five following chapters.

The first chapter describes usage and structure of contact calls -grunts, clear barks and loud calls- in two troops of olive baboons living in Gashaka-Gumti National Park, Nigeria. I focus on the contexts in which these vocalisations are uttered, on the rate at which they are emitted, and on some of their acoustic features. Results are discussed in the light of findings in other populations of olive baboons and in other baboon taxa. This chapter has two purposes. First, it describes vocalisations which will be the foci of following analyses in this thesis (chapters 4 and 5). Second, it provides data to document inter-population and intertaxon variability in baboon vocal communication.

The second chapter summarises background knowledge on environment-related variations in vocal communication in anurans, birds and mammals. I review studies investigating the influences of the environment on usage and structure of vocal signals in these taxa. Results of these studies are compared to general hypotheses based on processes implicated in sound propagation to draw conclusions. This chapter gathers the current evidence of adjustment (or lack thereof) in response to environmental conditions in usage and structure of vocalisations in anurans, birds and mammals.

The third chapter presents methods to characterise the environment before examining its effect on vocal communication, as suggested in Chapter 2. Structural aspects are characterised by tree density and visual vegetation density, while acoustic features are described by the level, frequency range and daily variations of ambient noise and by frequency-dependent attenuation profiles. The aim of this chapter is to provide some standardised methods for habitat characterisation and to underline the necessity of combining different methods for an accurate description of an environment. These methods are applied to describe the study site in Nigeria where studies of chapters 1, 4 and 5 are conducted.

In the fourth chapter, I examine whether wild female olive baboons adjust the acoustic structure and the emission rate of their contact calls used for short-distance communication (i.e., grunts) to the environment. Data collected in Gashaka-Gumti National Park, Nigeria, are combined with those collected by C. Rahn in Budongo Forest Reserve, Uganda, in another troop of olive baboon. Grunt rates and grunt acoustic structure are compared between forest (a closed habitat type) and woodland savannah with low grass (an open habitat type), as well as between high grass (another closed habitat type) and low grass within woodland savannah. Recording the same individuals in different environmental conditions while controlling call contexts provides the highest level of control of other potentially confounding factors. The 
aim of this chapter is to evaluate the degree of intra-individual flexibility in response to variations in environmental conditions in usage and production of calls used for short-distance communication.

The fifth chapter documents influences of environmental conditions on usage of contact calls used for long-distance communication (i.e., clear barks and loud calls) in olive baboons. I consider call rate of clear barks in the Nigerian and Ugandan troops and timing of loud calls in the two Nigerian troops. My aim is to examine whether baboons adjust these aspects of call usage to structural and acoustic characteristics of the habitat they range in.

At last, since results are largely discussed in the respective chapters, I comment only the two major findings of this thesis in a general conclusion. I also suggest directions for future investigations. And finally I briefly discuss some implications of the present thesis concerning the evolution of speech.

Overall, this thesis aims to evaluate the degree of flexibility at an intra-individual level in response to variations in environmental conditions in usage and structure of vocalisations used for short- and long-distance communication in olive baboons. In addition, it provides some data to discuss inter-population and inter-taxon variability in usage and structure of baboon contact calls. Therefore, this thesis contributes to a long-term project which should integrate various factors (phylogeny, social organisation, and environment) to examine their interplay in shaping baboon communication system. 


\title{
Chapter 1
}

\section{"Keep in touch" - Contact calls in olive baboons}

\begin{abstract}
Living in a group might provide essential advantages such as reducing predation risk, as well as increasing foraging and reproductive success. Vocal signals might have evolved in social primates (among other taxonomic groups) to maintain contact between group members and to monitor other groups. Contact calls used over short (grunts) and long (clear barks and loud calls) distances are shared by all baboon taxa. They are then appropriate to study the interplay of various factors (e.g., phylogeny, social system and environment) in the regulation of their usage and design of their structure. To broaden current knowledge on baboon vocal communication, the present chapter concentrates on the vocal behaviour of two troops of olive baboons living in Gashaka-Gumti National Park, Nigeria. The contexts in which contact calls are uttered, their emission rate, and some of their acoustic features are described. Results are then discussed in the light of findings in other olive baboon populations and in other baboon taxa. In the study population, contexts of grunts and clear barks corresponded to those previously described, whereas loud calls were not used in contest situations which are often reported in chacma baboons. Grunt rate did not vary within the Nigerian population but between populations of olive baboons and between baboon taxa. Bark rate differed within and between olive baboon populations, as well as between baboon taxa. In contrast, the acoustic structure of grunts, clear barks and loud call bouts presented only minor differences between populations and taxa. These results are in line with the prediction of a flexible call usage, as opposed to a more fixed call structure between populations or taxa. They additionally suggest that various factors such as proximity between group members or tendency for the troop to split and rejoin might regulate usage of contact calls. Future studies are needed to integrate these factors in order to understand their interplay in the design of a communication system essential in a group-living species.
\end{abstract}

This chapter was written in collaboration with Julia Fischer. Volker Sommer and Caroline Ross commented some parts of an earlier version. 


\section{$1.1 \quad$ INTRODUCTION}

Most primates are social animals. Despite a higher competition for resources, living in social groups presents non-negligible vital advantages, such as increasing foraging and reproductive success as well as enhancing survival by reducing predation risk (Rowell 1972; van Schaik 1983; Henzi \& Barrett 2003; summarised in Kappeler 2000). Therefore, maintaining bonds between conspecifics is essential. One way to "keep in touch" with other group members but also to monitor other groups is to use vocal signals. These contact vocalisations present the advantage of being effective over short and long distances. They occur in most primate vocal repertoires, especially in species moving in large, compact groups, in those in which groups subdivide into subgroups, and in those ranging in habitats with dense vegetation and poor visibility (Rowell 1972). According to whether they are used over long or short distances, contact calls used within groups serve to maintain contact, to regulate spacing and/or to facilitate social relationships between group members (e.g., Robinson 1982; Boinski 1991; Cheney et al. 1996; Palombit et al. 1999; Rendall et al. 1999). Since some of these vocalisations are also given when individuals are not in visual contact, they are generally characterised by prominent identity cues (at group and/or individual levels; e.g., Snowdon \& Cleveland 1980; Oda 2002; Rendall 2003; Braune et al. 2005). Contact calls used to regulate interactions and spacing between groups are loud vocalisations carrying over large distances. They may also contain group- and individual-specific signatures (e.g., Braune et al. 2005; Lameira \& Wich 2008). Inter-group communication is well developed in primates living in closed habitats, where vegetation obstructs visibility (e.g., Rowell 1972; Chivers 1976). These calls usually follow an encounter with another group, a group movement, a disturbance, other individuals' vocalisations, or they may be given spontaneously, that is, without obvious stimuli eliciting them (Waser 1977; Byrne 1982; Waser 1982; Rendall \& Owren 2002).

In primates, usage (e.g., contexts of calling, call rate) of vocal signals is known to be flexible. Variations occur between (sub)species or between populations and can be related to social organisation (Rowell 1972) or environment (Schneider et al. 2008) for instance. In contrast, the acoustic structure of vocal signals is considered to be mostly innate and phylogenetically constrained (see also General introduction). Nevertheless, some degree of variability between (sub)species or populations occurs, as a response to different factors. For instance, some factors linked to geographical distance might explain inter-population variability in shrill barks of Barbary macaques (Fischer et al. 1998) and pant hoots of 
chimpanzees (Pan troglodytes schweinfurthii: Mitani et al. 1999; Crockford et al. 2004). Genetic relatedness and social factors might also explain some variations. These characteristics of usage and structure concern, among other call types, contact vocalisations.

Baboons (genus Papio) are suitable to conduct an integrative study to understand the interplay of various factors such as social system or ecological conditions on the vocal communication system (General introduction). In this genus, some control on phylogenetic relationships between baboon taxa and populations seems now possible according to recent investigations (Zinner et al. 2008). Most importantly, all baboon taxa have similar contact calls, namely grunts, clear barks and loud calls. These vocalisations are therefore particularly relevant to document variability between baboon populations or taxa (General introduction). Grunts are used for short-distance communication (Figure 1.1a). These are short, quiet, lowpitched, and harmonically rich calls uttered during affiliative interactions, after distress calls from an infant or during resting and feeding and before a group movement (Rowell 1966; Ransom 1981; Owren et al. 1997; Rendall et al. 1999, 2004). They are given by all age and sex classes, except by very young infants (Ransom 1981). The structure of these calls presents clear identity cues (e.g., in formant frequencies and fundamental frequency: Rendall 2003) and varies according to the caller's size and therefore also its age and sex (Rendall et al. 2004; Pfefferle \& Fischer 2006). Clear barks -or contact barks- are used for long-distance communication (Figure 1.1b). These barks are loud and tonal, and given by animals separated from the rest of the group or from particular individuals (Rowell 1966; Byrne 1981; Ransom 1981; Cheney et al. 1996; Rendall et al. 2000; Fischer et al. 2001a, 2002). They are uttered by all age and sex classes (Ransom 1981), and their acoustic structure varies accordingly (Ey et al. 2007b). Loud calls are also presumably used for long-distance communication (Figure 1.1c). These bouts of loud two-phased barks -termed "wahoos"- are given only by adult males when they are herding females during group encounters, after males of other groups have uttered some wahoos (especially at night), before water crossing or spontaneously (Buskirk et al. 1974; Ransom 1981; Fischer et al. 2002). These calls are also given by a male after he chased a group member (Ransom 1981) and in contest situations with other males of the group (Fischer et al. 2002). Bouts of these barks might be preceded by a roar or a hum and a roar (Hall \& de Vore 1965; Ransom 1981). In chacma baboons, contest bark acoustic features vary according to the rank of the caller and might reflect its competitive ability (Fischer et al. 2002; Kitchen et al. 2003b; Fischer et al. 2004). 
a.

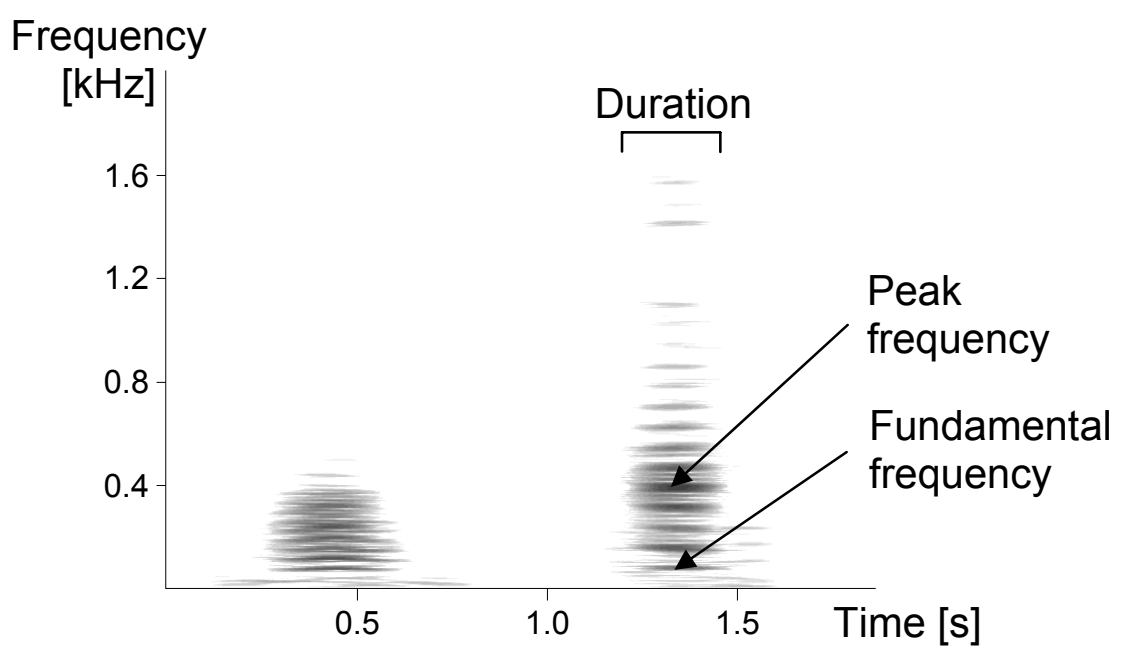

b.

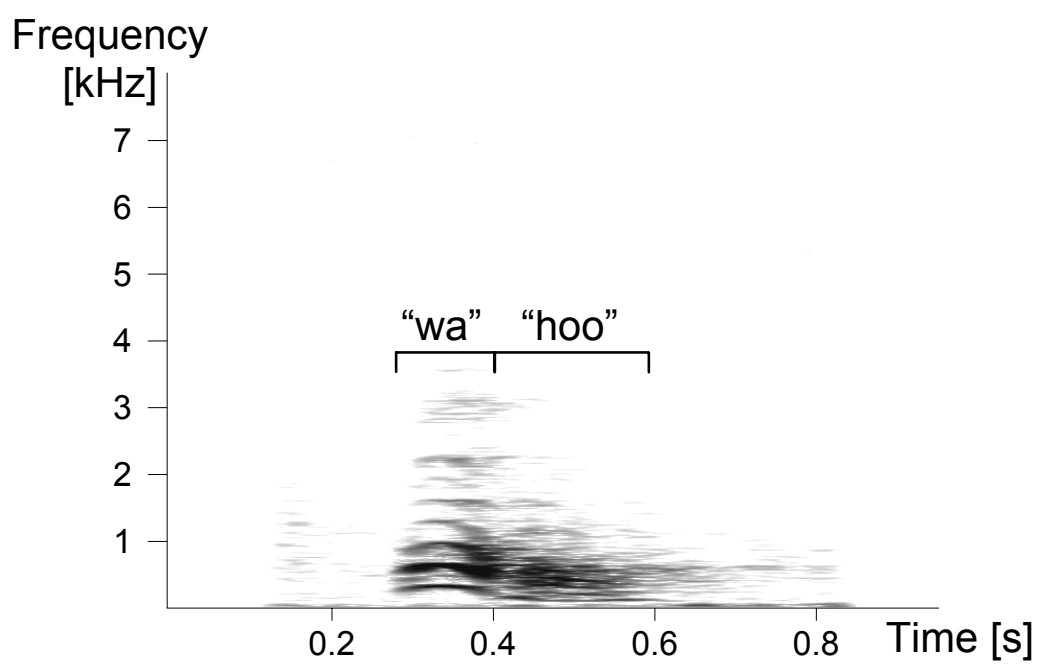

c.

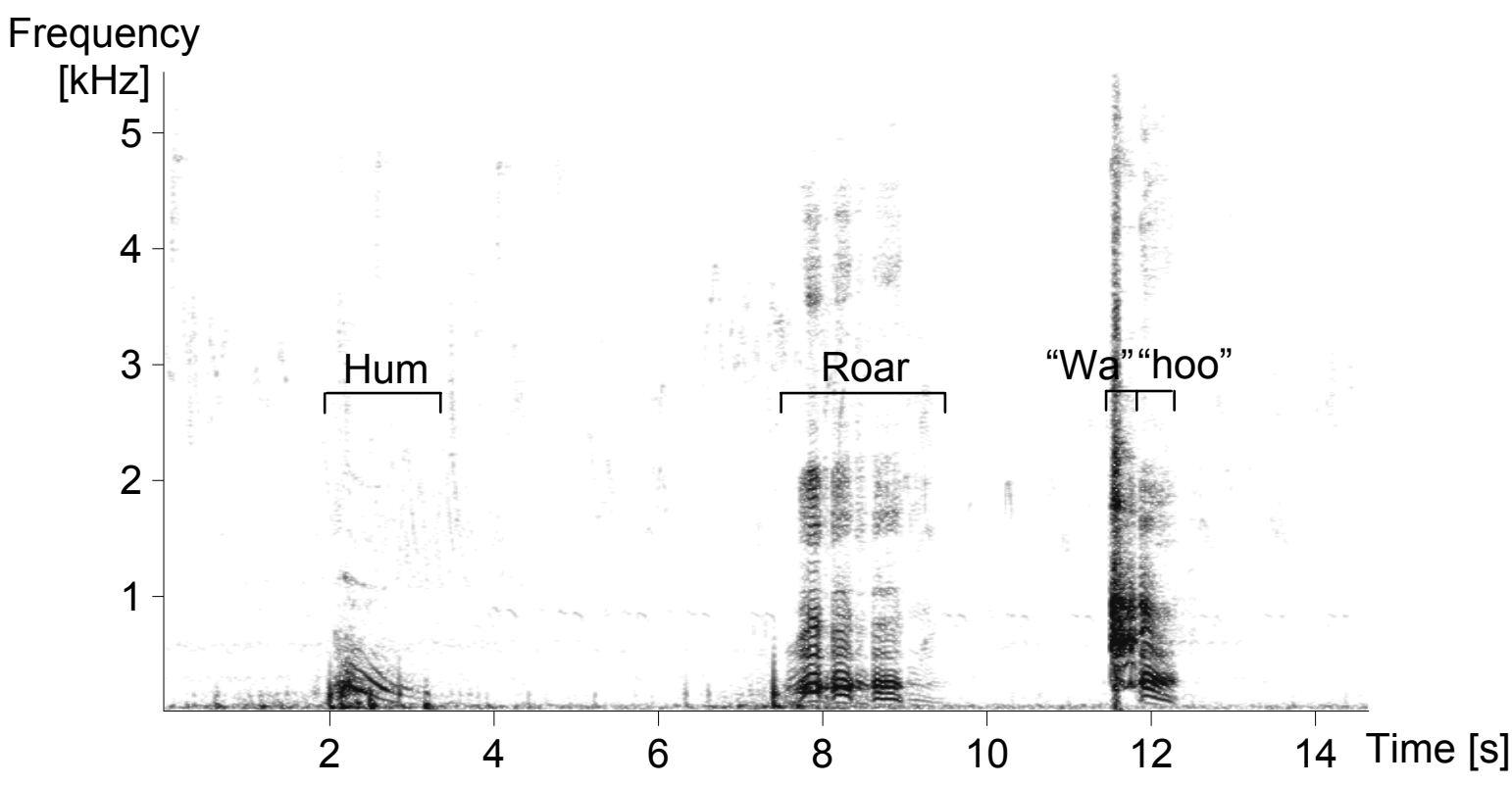

Figure 1.1: Spectrograms of contact vocalisations in adult olive baboons. (a) Grunt (left: male; right: female). (b) Clear bark, also termed contact bark (female). (c) Beginning of a loud call bout (male). 
So far, contexts of emission and acoustic structure of grunts, clear barks, and loud calls were mostly studied in chacma baboons. To broaden knowledge on baboon vocal communication, the present chapter describes usage and structure of these calls in two troops of olive baboons ranging in Gashaka-Gumti National Park, Nigeria. It documents the contexts in which these vocalisations are uttered, their general emission rate (and its variations with the caller's sex), and some general acoustic features (i.e., duration, fundamental frequency and peak frequency; Figure 1.1a, see methods for definitions). These frequently-used acoustic variables were selected to facilitate comparisons with data from other baboon populations. All results are discussed in the light of findings in other olive baboon populations and other baboon taxa.

Since call usage is usually more flexible than call structure (see above), the former is expected to show a higher level of variability between populations or taxa than the later. Variability between taxa or between populations which have diverse phylogenetic, ecological or social characteristics is expected in contexts and emission rates of contact vocalisations. In contrast, the acoustic structure of these vocalisations is considered to be phylogenetically constrained and should therefore present only minor differences within the genus Papio.

\subsection{MATERIAL AND METHODS}

\subsubsection{Study site and subjects}

Data were collected in the southern sector of Gashaka-Gumti National Park, eastern Nigeria, near Cameroon. The area is located in the Guinea savannah zone, but nevertheless includes a mosaic of vegetation types, such as southern Guinea savannah (hereafter woodland savannah), lowland rainforest and riverine forest. These two forest variants are referred to as forest hereafter. Woodland savannah is regularly burnt (Dunn 1993; Harcourt \& Ellerton 1995; Akinsoji 1996; Chapman \& Chapman 2001). Chapter 2 provides a more precise description of the study site.

Two troops of olive baboons were studied over a total of 10 months distributed over two dry seasons (Nov - Dec 05, Feb - May 06, Nov 06 - Apr 07), since audio-recordings were difficult during times of heavy rainfall (Jul - Oct). The Gamgam troop range is located along Gamgam river near Gashaka village, just outside Gashaka-Gumti National Park. It consists of woodland savannah, with narrow bands of riverine forest along seasonal streams. This troop supplements its diet by raiding crops, leading to regular chases by farmers (Warren 2003). 
The Kwano troop ranges within Gashaka-Gumti National Park. Lowland rainforest largely covers its range, which also includes patches of woodland savannah. This troop has little interaction with human beings, except field assistants and researchers (Warren 2003). Baboons of the two troops range in both forest and woodland savannah during the day. Both troops have been fully habituated to researchers since 2000, allowing observation of adults and subadults from a $2-6 \mathrm{~m}$ distance. Infants and juveniles could usually not be approached to less than $5 \mathrm{~m}$. The size and composition of the troops varied slightly over the observation period, with 20 - 21 individuals in Gamgam troop and 24 - 29 individuals in Kwano troop (Table 1.1). Phylogenetic reconstruction based on mitochondrial DNA extracted from faecal samples indicates that the two troops share the same haplotypes, suggesting that members have a common matrilineal ancestry (Zinner et al., in press).

Table 1.1: Composition of Gamgam and Kwano troops in the study period Nov - Dec 05, Feb - May 06, and Nov 06 - Apr 07.

\begin{tabular}{l|c|c} 
& Gamgam & Kwano \\
\hline Adult males & $1-3$ & $3-5$ \\
Adult females & 5 & 9 \\
Subadult males & 0 & $1-2$ \\
Subadult females & 0 & 1 \\
Juveniles & $6-8$ & $4-6$ \\
Infants & $6-8$ & $5-9$
\end{tabular}

\subsubsection{Data collection}

Each day, one focal animal was followed from 06:00 to 12:00. Focal observations rotated between 6 animals in Gamgam troop ( 1 adult male, 5 adult females) and 13 in Kwano troop ( 3 adult males, 10 adult females). Males were considered adult when they had fully developed secondary sexual characters (canines and shoulder mane) and when they were sexually active in the troop. Females were classified as adult at their first pregnancy (among the 10 focal females in Kwano, one became pregnant only early 2007 but was regularly cycling since the beginning of the study). Every $15 \mathrm{~min}$, habitat type, activity of the focal animal, and number of group members within $10 \mathrm{~m}$ of the focal animal were recorded in a scan. The factor habitat type was introduced in some analyses of behavioural data because it was suspected to influence group spread and activity distribution (when not specified, data from all habitat types were considered without distinction). Activities were separated in two categories ("non-interactive" and "interactive") which paralleled the "social" and "non-social" ones often found in the literature. Non-interactive activities included foraging (actively 
foraging or feeding from a food source), resting (sitting, standing or lying; with two subcategories: resting within $2 \mathrm{~m}$ of another individual and resting alone), and travelling (walking, running, climbing in trees). In these non-interactive situations, the focal animal was not directly interacting with other group members. In contrast, interactive activities included all direct social interactions, such as friendly approaches towards individuals (the outcome was either sitting near the other one, passing its way, sitting alone as the other one left, or interacting with the other one), infant handling (this activity was recorded either for the handler or the mother of the handled infant), grooming / being groomed, embracing / being embraced, presenting/ receiving a presentation, mounting/being mounted, and aggressing/being aggressed. Vocal signals uttered in interactive activities were clearly directed to another individual, whereas those uttered in non-interactive activities could be either directed to another baboon or undirected (i.e., without obvious recipient). It should be noted that activity scans of the focal animal recorded mostly long-lasting states. For instance, the behaviours "approach", "embrace", "present", and "mount" did not appear in the scans because they are events, but they occurred in the contexts of grunts.

All grunts from the focal animal were recorded, as well as grunts from other individuals whenever caller identity and context could be determined. Clear barks and loud calls, as they were uttered more rarely, were recorded ad libitum from all group members. Time, caller identity, context, habitat type, and height of call post were noted for each vocalisation. Audio-recordings were made using a Marantz PMD660 solid-state recorder (Marantz, Japan; $44.1 \mathrm{kHz}$ sampling frequency, 16-bit resolution, mono) and a Sennheiser directional microphone (K6 power module and ME66 recording head, with a Rycote softie windscreen; Sennheiser Electronic KG, Germany). Audio-recordings were interrupted when acoustic conditions were not good enough, for instance when the troop stayed in a river bed, as background noise from water covered the frequency range of vocalisations and therefore the signal-to-noise ratio was not strong enough.

Over the two field seasons, a total of 6124 and 4064 grunts were recorded from adult animals in $370 \mathrm{~h}$ and $455 \mathrm{~h}$ of observation in Gamgam troop and Kwano troop, respectively. Among these, 3016 grunts in Gamgam troop and 2141 grunts in Kwano troop were recorded from the focal animals in their respective focal follows. These data were used to examine the contexts in which grunts were uttered and grunt rates. An "individual" grunt rate was calculated by counting all single grunts (i.e., the units in bouts) uttered by the focal animal over the total recording time for this individual. 
Since clear barks were given much more rarely -and usually not by the focal animal-, a "group" bark rate was estimated. All clear barks within earshot (even from unidentified callers) were counted (Gamgam: 218; Kwano: 832) and related to daily observation time. This represents an estimation of how many calls a baboon might hear, since the main energy of barks $(0.3-10 \mathrm{kHz})$ falls within the hearing range of baboons (Papio cynocephalus: 0.04 $40 \mathrm{kHz})$ and humans $(0.03-17.6 \mathrm{kHz}$; Heffner 1998; Heffner 2004). Bark rates were obtained for 57 and 66 days in Gamgam and Kwano troops, respectively.

Loud calls were recorded only in Nov 06 - Apr 07 (systematically in Dec 06 - Apr 07). A "group" bout rate was calculated, by dividing the number of bouts heard (Gamgam: 27 bouts; Kwano: 26 bouts) by the observation time (Gamgam: 168.5 h; Kwano: $184.2 \mathrm{~h}$ ). The within-bout bark rate was calculated in two ways (the aim was to check whether the two ways of calculation give equivalent rates). First, the number of wahoos within a bout was divided by the total duration of the bout, from the onset of the first vocalisation (hum, roar, or wahoo) to the offset of the last one (wahoo). This rate could be calculated in 23 completed bouts (Gamgam: 17; Kwano: 6). Second, the within-bout bark rate was calculated as the mean of the bark rate in each inter-bark interval (i.e., from the onset of a bark to the onset of the next one; see Fischer et al. 2002). This bark rate could be calculated in 15 of the 23 completed bouts containing more than one wahoo and of sufficient quality (limited background noise) to determine precisely all inter-bark intervals.

\subsubsection{Acoustic analyses}

\subsubsection{Definition of acoustic variables}

Sound production involves inter-costal muscles which contract themselves, so that the volume of the rib cage is reduced. Air is then forced from the lungs into the trachea. The amount of expulsed air and its speed determine sound duration. The air flow passes through the vocal folds, which begin to oscillate. The fundamental frequency of a sound is that at which the vocal folds are vibrating and depends on their tension, mass and elasticity. During tonal sound production, the source signal is comprised of the fundamental frequency and its multiple integers (harmonics). The sound waves then pass through the vocal tract (between the glottis and the opening of mouth or nose) until they emanate. Depending on the shape and length of the vocal tract, different frequencies among the harmonics may either be filtered or enhanced. The resulting frequency spectrum thus depends on the source signal and the filter function, typically resulting in different formants (i.e., peaks in the frequency spectrum; see 
Fitch \& Hauser 1995 for a review). The peak frequency is the frequency containing the highest energy. Fundamental and peak frequencies are calculated for each time segment within a call, and the means are then calculated over all time segments.

\subsubsection{Grunts}

Among all grunts recorded (grunts recorded from non-focal adult animals were also included), 1853 (Gamgam) and 1044 (Kwano) were of sufficient quality (i.e., not disturbed by noise of wind, insects, birds or other baboons). Only grunts recorded from a distance of 3 $12 \mathrm{~m}$ were used for the acoustic analyses, since calls might be distorted over longer distances (Fischer et al. 2002). This data set was further reduced to obtain an even distribution between callers, contexts and habitat types. This last factor was introduced because the environment was suspected to have an influence on grunt structure (see Chapter 4) and only grunts uttered in forest and in woodland savannah were included. Thus, 2 - 11 grunts per animal per habitat type (same number of grunts in forest and woodland savannah) and per activity category (interactive / non-interactive) were selected from 4 males and 8 females. This led to a final set of 342 grunts (interactive: 93 grunts per habitat type; non-interactive: 78 grunts per habitat type). Grunt acoustic structure was also compared between more precisely defined contexts (interactive: infant handling; non-interactive: resting alone). These two specific contexts were chosen in order to be compared with results in Owren et al. (1997) and Meise (2008). Recordings from 6 females (7-48 grunts per animal) in both habitat types and in both contexts (infant handling: 59 grunts; resting alone: 92 grunts) were selected.

Sampling frequency was lowered from $44100 \mathrm{~Hz}$ to $5512.5 \mathrm{~Hz}$ to obtain a higher frequency resolution in the frequency range of grunts, using the software Avisoft SASLab Pro Recorder 4.3 (R. Specht; Berlin, Germany). Duration was measured manually on the first harmonic using the standard cursor function on spectrograms calculated by the same software (sampling frequency: $5512.5 \mathrm{~Hz}$; FFT-length: 1024 points; Hamming window; overlap: $98.43 \%$; time resolution: $2.9 \mathrm{~ms}$; frequency resolution: $10.8 \mathrm{~Hz}$ ). Binary spectrograms were saved and files were exported into the software LMA 2005 developed by K. Hammerschmidt (Schrader \& Hammerschmidt 1997). The harmonic cursor tool was used to calculate mean fundamental frequency and mean peak frequency for each grunt (start and end amplitude thresholds: $10 \%$; cut-off frequency: set under the fundamental frequency and as far as possible above background noise [i.e., according to the call at $35 \mathrm{~Hz}, 42 \mathrm{~Hz}, 50 \mathrm{~Hz}, 58 \mathrm{~Hz}$, or $70 \mathrm{~Hz}$; noise factor: 1.5; repetition factor: 1; amplitude calculation mode: general; no filter; contour threshold: 0.2; F0 modulation limit: $30 \%$; F0 range for IM: 0.5). The distributions of 
all values for each acoustic variable were checked afterwards and potential outliers were corrected. When fundamental frequency could not be detected, or the calculation of peak frequency was disturbed by extraneous noise, calls were replaced or excluded from the analyses.

\subsubsection{Clear barks}

Among all clear barks heard, 131 and 368 were recorded in Gamgam and Kwano troops, respectively. Among these recordings, 31 and 89 were of sufficient quality for acoustic analyses, but, as for grunts, the data set was further reduced to obtain a more even distribution. Here, 28 clear barks from 11 adult females ( 1 - 5 barks per female) constituted the final set. Sampling frequency was lowered from $44100 \mathrm{~Hz}$ to $8000 \mathrm{~Hz}$ to obtain a higher frequency resolution in the frequency range of clear barks, using the software Avisoft. The binary spectrograms (sampling frequency: $8000 \mathrm{~Hz}$; FFT-length: 1024 points; frequency resolution: 15.7 Hz; Hamming window; overlap: $96.87 \%$; time resolution: $4 \mathrm{~ms}$ ) were exported from Avisoft to LMA 2005. The general macro of this software (cut-off frequency: $150 \mathrm{~Hz}$; start and end amplitude thresholds: $5 \%$; other settings: see above) was used to estimate duration of whole calls ("wa" and "hoo" parts together). The harmonic cursor tool (cut-off frequency: $150 \mathrm{~Hz}$; start and end amplitude thresholds: $5 \%$; other settings: see above) was used to calculate mean fundamental frequency and mean peak frequency on the "wa" part of the calls.

\subsubsection{Loud calls}

Among all loud call bouts heard, 20 and 7 were recorded in Gamgam and Kwano troops, respectively. Sampling frequency was left at $44100 \mathrm{~Hz}$ to describe bout structure. Time resolution was high enough to measure temporal parameters. Duration of bouts and inter-bark intervals (to calculate within-bout call rates) were measured on the envelope curve of the main window of the software Avisoft with the marker function. No other acoustic variable was measured.

\subsubsection{Statistical Analyses}

The distribution of interactive activities between forest and woodland savannah was tested with exact 2-tailed Wilcoxon T-tests in each troop separately. Exact 2-tailed MannWhitney U-tests were used to compare group spread (i.e., number of individuals within $10 \mathrm{~m}$ 
of the focal animal) between troops, grunt rates between troops and between sexes and bark rates between troops. The two ways of calculating within-bout bark rate for loud calls were compared using an exact 2-tailed Wilcoxon T-test in the 15 bouts for which both were available. Non-parametric tests were used in these analyses because of small sample sizes (Mundry \& Fischer 1998). To investigate the effect of the activity category (interactive / noninteractive) and habitat type (forest/ woodland savannah) on group spread, a linear mixed model was used with activity category, habitat type and the interaction activity category $\times$ habitat as fixed factors in each troop separately (since the troops differed in size). A linear mixed model with activity category as a fixed factor was used to test the influence of the activity on grunt acoustic structure (duration, mean fundamental frequency, mean peak frequency). In this model, p-values were corrected for multiple testing (the same model was used for each acoustic variable) with a Step-up Hochberg correction (Westfall \& Young 1993). For a more precise comparison of acoustic variables of directed grunts given in the interactive context of infant handling and undirected grunts given in the non-interactive context of resting alone, a linear mixed model was used, with activity as a fixed factor. A Step-up Hochberg correction for multiple testing was also used in this model. A linear mixed model with sex as a fixed factor was used to compare grunt acoustic characteristics between males and females. As in the two previous models, p-values were corrected for multiple testing with a Step-up Hochberg correction. In all linear mixed models, the identity of the focal animal (for behavioural data) or of the caller (for audio-recordings) was considered as a random factor. The software SPSS 15.0 and 16.0 for Windows was used for all statistical analyses and the significance level was set at 0.05 .

\subsection{RESULTS}

\subsubsection{Behavioural data}

Mean $( \pm \mathrm{SD})$ group spread within $10 \mathrm{~m}$ of the focal animal was similar in Gamgam troop $(1.80 \pm 0.29$ individuals / scan $)$ and in Kwano troop (1.93 \pm 0.54 individuals / scan; exact Mann-Whitney U-test: $\mathrm{N}_{1}=6, \mathrm{~N}_{2}=13, \mathrm{U}=32, \mathrm{p}=0.579$ ). Both troops were significantly more scattered in non-interactive activities than in interactive activities (linear mixed model: Gamgam: $F=23.87, p<0.001$; Kwano: $F=33.51, p<0.001$; Table 1.2). The effect of the interaction habitat type $\times$ activity on group spread was not significant, in any of the two troops. A significant effect of the habitat type on group spread was found in Gamgam 
troop (linear mixed model: $\mathrm{F}=21.07, \mathrm{p}<0.001$ ), but not in Kwano troop (linear mixed model: $\mathrm{F}=0.01, \mathrm{p}=0.908)$. Gamgam baboons were more scattered in woodland savannah than in forest (Table 1.2). This might be related to the fact that Gamgam baboons tended to spend more time socialising (i.e., in direct social interactions) in forest (mean $\pm \mathrm{SD}$ : $16.0 \pm 10.0 \%$ of scans in forest) than in woodland savannah (mean \pm SD: $9.0 \pm 4.8 \%$ of scans in woodland savannah), but this difference was not significant (exact Wilcoxon T-test: $\mathrm{N}=6, \mathrm{~T}=3, \mathrm{p}=0.156$ ). Kwano baboons did not spend significantly more time socialising in forest (mean \pm SD: $10.6 \pm 7.2 \%$ of scans in forest) than in woodland savannah (mean \pm SD: $10.0 \pm 6.4 \%$ of scans in woodland savannah; exact Wilcoxon T-test: $\mathrm{N}=13, \mathrm{~T}=33$, $\mathrm{p}=0.677)$.

Table 1.2: Mean and standard deviation of the number of individuals within $10 \mathrm{~m}$ of the focal animal per scan in interactive and non-interactive activities in forest and woodland savannah in Gamgam and Kwano troops.

\begin{tabular}{l|cc|cc} 
& \multicolumn{2}{|c|}{ Interactive } & \multicolumn{2}{c}{ Non-interactive } \\
& Forest & $\begin{array}{c}\text { Woodland } \\
\text { savannah }\end{array}$ & Forest & $\begin{array}{c}\text { Woodland } \\
\text { savannah }\end{array}$ \\
\hline Gamgam & $3.42 \pm 0.89$ & $2.41 \pm 0.56$ & $2.35 \pm 0.27$ & $1.53 \pm 0.34$ \\
Kwano & $3.10 \pm 0.83$ & $2.97 \pm 0.80$ & $1.73 \pm 0.59$ & $1.88 \pm 0.95$
\end{tabular}

Baboons spent about a tenth of their time in interactive activities (Gamgam: $11.3 \%$ of scans; Kwano: $10.8 \%$ of scans). Among these, grooming was most frequently observed, followed by infant handling (Table 1.3a). The remaining time was spent in non-interactive activities (Gamgam: $88.7 \%$ of scans; Kwano: $89.2 \%$ of scans). Among these, foraging and resting (within $2 \mathrm{~m}$ of a conspecific and alone) were most frequent; the rest of the time was spent in travelling (Table 1.3b). 
Table 1.3: Proportion (\%) of activity scans, and number (\#) and proportion (\%) of grunts in (a) interactive and (b) non-interactive activities in Gamgam and Kwano troops.

\begin{tabular}{|c|c|c|c|c|c|c|c|c|}
\hline & & Approach & $\begin{array}{c}\text { Handle } \\
\text { infant }\end{array}$ & Groom & Embrace & Present & Mount & Aggression \\
\hline \multirow{3}{*}{ Gamgam } & $\%$ scans & & 4.9 & 92.2 & & & & 2.9 \\
\hline & \# directed grunts (1093) & 491 & 467 & 63 & 66 & 6 & 0 & 0 \\
\hline & $\%$ directed grunts & 44.9 & 42.7 & 5.8 & 6.0 & 0.5 & 0.0 & 0.0 \\
\hline \multirow{3}{*}{ Kwano } & $\%$ scans & & 16.9 & 79.4 & & & & 3.7 \\
\hline & \# directed grunts (1061) & 382 & 593 & 50 & 7 & 16 & 13 & 0 \\
\hline & $\%$ directed grunts & 36.0 & 55.9 & 4.7 & 0.7 & 1.5 & 1.2 & 0.0 \\
\hline
\end{tabular}

\begin{tabular}{l|l|cccc}
\multicolumn{2}{l|}{} & Forage & Rest alone & Rest 2 m & Travel \\
\hline \multirow{5}{*}{ Gamgam } & \% scans & 46.2 & 21.6 & 12.2 & 20 \\
& \# directed grunts (960) & 178 & 506 & 161 & 115 \\
& \% directed grunts & 18.5 & 52.7 & 16.8 & 12.0 \\
& \# undirected grunts (755) & 215 & 430 & 0 & 110 \\
& \% undirected grunts & 28.5 & 56.9 & 0.0 & 14.6 \\
\hline \multirow{5}{*}{ Kwano } & \% scans & 53.8 & 21.6 & 9.2 & 17.1 \\
& \# directed grunts (594) & 194 & 218 & 80 & 102 \\
& \% directed grunts & 32.6 & 36.7 & 13.5 & 17.2 \\
& \# undirected grunts (300) & 141 & 138 & 0 & 21 \\
& \% undirected grunts & 47.0 & 46.0 & 0.0 & 7.0
\end{tabular}




\subsubsection{Grunts}

\subsubsection{Occurrences of grunts}

Grunts were given by all age and sex classes (rarely by young infants), but only grunts from adults were considered here because continuous data were collected only on this age class. These vocalisations were either given as single calls or as rapid series which might be followed by grunts from other individuals. In interactive situations, grunts were always directed to another individual. Direct social interactions elicited $38.9 \%$ of 2808 grunts recorded in Gamgam troop from 6 focal individuals and $54.3 \%$ of 1955 grunts recorded in Kwano troop from 13 focal individuals. Most interactive grunts were emitted during friendly approaches (Gamgam: 44.9\%; Kwano: 36.0 \%), and infant handling (Gamgam: 42.7\%; Kwano: $55.9 \%$ ). These two activities were either absent or underrepresented in activity scans, in contrast to grooming which was over-represented, but which elicited fewer grunts (Table 1.3a). In non-interactive situations, grunts clearly directed to a recipient and those apparently not addressed to any animal in the vicinity were separated. Most grunts -both directed and undirected- were uttered during foraging and resting. Fewer grunts were given during travelling (Table 1.3b).

\subsubsection{Grunt rate}

Mean $( \pm \mathrm{SD})$ grunt rates were higher in Gamgam troop $(0.29 \pm 0.11$ grunts / min $)$ than in Kwano troop $(0.19 \pm 0.08$ grunts / $\mathrm{min})$, although this difference was not significant (exact Mann-Whitney U-test: $\mathrm{N}_{1}=6, \mathrm{~N}_{2}=13, \mathrm{U}=18.0, \mathrm{p}=0.072$ ). In both troops, males (4 males $[$ mean $\pm \mathrm{SD}]: 0.26 \pm 0.05$ grunts $/ \mathrm{min}$ ) tended to have a slightly higher grunt rate than females (15 females [mean $\pm \mathrm{SD}$ ]: $0.22 \pm 0.11$ grunts / min), but again this difference did not reach significance (exact Mann-Whitney $\mathrm{U}$-test: $\mathrm{N}_{1}=4, \mathrm{~N}_{2}=15, \mathrm{U}=19.0, \mathrm{p}=0.307$ ).

\subsubsection{Acoustic characteristics of grunts}

The activity category (interactive / non-interactive) as defined in the present study did not have an influence on duration (linear mixed model: $F=1.26$, corrected $p=0.321$ ), mean fundamental frequency $(\mathrm{F}=1.14$, corrected $\mathrm{p}=0.321)$ or mean peak frequency $(\mathrm{F}=3.34$, corrected $p=0.110$ ). However, when more precisely defined contexts (infant handling vs. resting alone) were compared, directed grunts given during infant handling were on average $( \pm \mathrm{SD})$ longer $(189 \pm 33 \mathrm{~ms})$ than undirected grunts given while resting alone $(166 \pm 29 \mathrm{~ms}$; 
linear mixed model: $\mathrm{F}=20.46$, corrected $\mathrm{p}<0.001$ ). This effect was not significant in mean fundamental frequency or mean peak frequency.

There was a significant effect of sex in all three acoustic variables. Males emitted longer grunts (linear mixed model: $\mathrm{F}=12.67$, corrected $\mathrm{p}=0.005$ ), with a lower mean fundamental frequency $(\mathrm{F}=35.01$, corrected $\mathrm{p}<0.001)$ and a lower mean peak frequency $(\mathrm{F}=20.66$, corrected $\mathrm{p}=0.002)$ than females (Table 1.4, first two columns; Figure 1.1a).

Table 1.4: Means and standard deviations of duration, mean fundamental frequency (F0 mean) and mean peak frequency (Pf mean) in grunts of adult baboons.

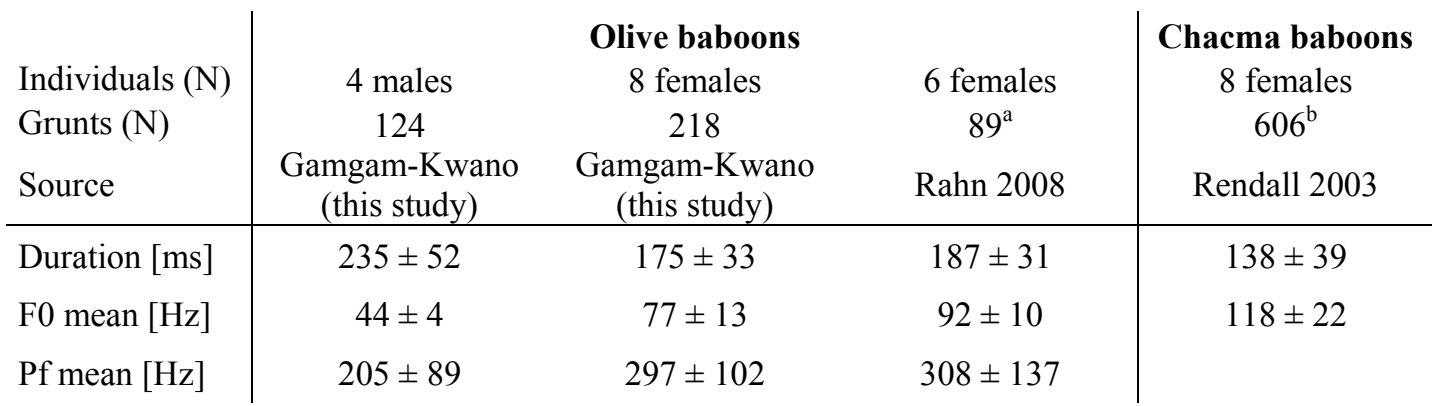

${ }^{\mathrm{a}}$ Undirected grunts in non-interactive contexts. ${ }^{\mathrm{b}}$ Grunts during infant handling and before a group movement.

\subsection{3 $\underline{\text { Clear barks }}$}

\subsubsection{Occurrences of clear barks}

Most clear barks occurred as single calls (Gamgam: 69.4\%; Kwano: 65.9\%), separated by more than $5 \mathrm{~min}$ from any other bark of the same animal. The remaining calls were given in bouts of 2 - 10 calls (except for one bout comprising 33 calls in Kwano troop when a mother had lost contact with her infant).

In the following analyses, only barks from immature animals and adult females for which caller identity was known were considered. Most clear barks were uttered when females lost contact with their infants or vice versa, but sometimes also by individuals who were alone or in a subgroup separated from the rest of the group. Usually, animals looked around as they vocalised. Some barks were uttered from perches on trees or rocks. Perched calls were more common in Kwano troop (immature animals: $47.7 \%$ of 44 barks, adult females: $44.8 \%$ of 181 barks) than in Gamgam troop (immature animals: $10.0 \%$ of 10 barks, adult females: $35.3 \%$ of 51 barks). Clear barks were uttered in non-interactive activities (Table 1.5). 
Table 1.5: Contexts of occurrences of clear barks from adult females and immature animals in Gamgam and Kwano troops.

\begin{tabular}{ll|c|cccc|c} 
& Troop & $\begin{array}{c}\text { Individuals } \\
(\mathrm{N})\end{array}$ & Forage & $\begin{array}{c}\text { Rest } \\
\text { alone }\end{array}$ & $\begin{array}{c}\text { Rest } \\
2 \mathrm{~m}\end{array}$ & Travel & $\begin{array}{c}\text { Unknown } \\
\text { context }\end{array}$ \\
\hline \multirow{2}{*}{ Adult females } & Gamgam & 4 & 14 & 11 & 0 & 8 & 18 \\
& Kwano & 10 & 46 & 74 & 1 & 48 & 12 \\
\hline \multirow{2}{*}{ Immature animals } & Gamgam & 5 & 0 & 5 & 0 & 3 & 2 \\
& Kwano & 9 & 0 & 18 & 0 & 22 & 4
\end{tabular}

\subsubsection{Bark rate}

Mean $( \pm \mathrm{SD})$ bark rate in Gamgam troop $(1.03 \pm 2.75$ barks / h, or corrected for troop size: $0.05 \pm 0.13$ barks $/ \mathrm{h} /$ individual) was significantly lower than in Kwano troop $(2.93 \pm 4.55$ barks / h, or corrected for troop size: $0.11 \pm 0.16$ barks / h / individual; exact Mann-Whitney U-test: $\left.\mathrm{N}_{1}=57, \mathrm{~N}_{2}=66, \mathrm{U}=969.0, \mathrm{p}<0.001\right)$.

\subsubsection{Acoustic characteristics of clear barks}

Clear barks constituted of two parts: a tonal, relatively high-pitched first part ("wa"), and a second one ("hoo"), which was quieter, noisier and lower pitched (Figure 1.1b). General acoustic features of clear barks were calculated for adult females (Table 1.6, first column).

Table 1.6: Means and standard deviations of duration, mean fundamental frequency (F0 mean) and mean peak frequency (Pf mean) of clear barks of adult females. Duration was measured over whole calls ("wa" and "hoo" parts), but mean fundamental frequency and mean peak frequency were measured on the "wa" part only.

\begin{tabular}{|c|c|c|}
\hline & Olive baboons & Chacma baboons \\
\hline Individuals (N) & 11 & 22 \\
\hline Clear barks $(\mathrm{N})$ & 28 & 39 \\
\hline Source & $\begin{array}{c}\text { Gamgam-Kwano } \\
\text { (this study) }\end{array}$ & $\begin{array}{l}\text { Fischer et al. } 2001 \mathrm{a} \\
\text { Ey et al. 2007b }\end{array}$ \\
\hline Duration [ms] & $407 \pm 101$ & $373 \pm 69$ \\
\hline F0 mean $[\mathrm{Hz}]$ & $435 \pm 58$ & $471 \pm 54$ \\
\hline Pf mean $[\mathrm{Hz}]$ & $751 \pm 175$ & $936 \pm 178$ \\
\hline
\end{tabular}

\subsubsection{Loud calls}

\subsubsection{Occurrences of loud calls}

Twenty-seven and twenty-six bouts of loud calls were heard in Gamgam and Kwano troops, respectively. These calls were given by adult males in bouts of 1 - 25 barks. Only one individual was calling at a time.

The contexts of calling could be clearly determined in 12 (Gamgam) and 8 (Kwano) bouts. These calls were given by animals sitting in a tree or on a rock without obvious 
stimulus eliciting vocalising, after they had chased a group member (an adult female or a juvenile), after a male of another group had uttered a bout, during an inter-group encounter, or more rarely (in Gamgam only) after being chased by farmers, after a scream from a juvenile, or before entering elephant grass (Table 1.7). The longest bouts (between 5 and 25 barks) were uttered after a bout from a male of another troop or without obvious stimuli. Bouts given after chasing a group member contained never more than two barks.

Table 1.7: Contexts of occurrences of loud call bouts in Gamgam and Kwano troops.

\begin{tabular}{l|c|c} 
& Gamgam troop & Kwano troop \\
\hline Without obvious stimulus & 4 & 3 \\
After chasing a group member & 4 & 3 \\
After loud calls in another group & 1 & 1 \\
Inter-group encounter & 0 & 1 \\
Being chased by farmers & 1 & 0 \\
After a scream of a group member & 1 & 0 \\
Before entering elephant grass & 1 & 0
\end{tabular}

Call post could be determined in 11 cases in Gamgam troop and 7 cases in Kwano troop. Loud calls were emitted from perches on trees or rocks in 8 occurrences in Gamgam troop and 5 occurrences in Kwano troop. Otherwise, they were uttered from the ground, especially after chasing another group member.

\subsubsection{Loud call rates}

The overall bout rates for all adult males together (Gamgam: 1; Kwano: 3 - 5) were 0.16 and 0.14 bouts / $\mathrm{h}$ (or corrected for the mean number of males: 0.16 and 0.04 bouts $/ \mathrm{h}$ ) in Gamgam and Kwano troops, respectively. Within bouts, wahoos were given at a mean $( \pm \mathrm{SD})$ rate of $8.01 \pm 5.09 \mathrm{barks} / \mathrm{min}$ (mean calculated over whole bout). The mean $( \pm \mathrm{SD})$ within-bout bark rate calculated over inter-bark intervals was $8.85 \pm 5.43 \mathrm{barks} / \mathrm{min}$. These two ways of calculation did not lead to significantly different rates (exact Wilcoxon T-test: $\mathrm{N}=15, \mathrm{~T}=29, \mathrm{p}=0.083$ ).

\subsubsection{Acoustic characteristics of loud calls}

Most recorded bouts began with a roar $(55.6 \%$ of 27 bouts) or a hum and a roar ( $37.0 \%$ ); the remaining 2 bouts began with a bark. In 9 of the 25 bouts containing a roar, a second roar followed the first bark and in one case a third roar followed the second bark. Barks were composed of two parts: a loud "wa" part, and a second quieter and lower pitched 
"hoo" part (Figure 1.1c). Not enough good-quality bouts from different animals were recorded to conduct any acoustic analyses.

\subsection{DISCUSSION}

The present chapter aimed to broaden knowledge on baboon vocal communication. It presented the contexts and rates of emission as well as some acoustic features of grunts, clear barks and loud calls in olive baboons. Many grunts were uttered in interactive activities, despite the fact that these represented a limited proportion of the time budget. Grunt rates were similar between the two troops and between sexes. Grunt acoustic structure did not differ between gross activity categories (interactive vs. non-interactive), but it did so between more precisely defined contexts (infant handling vs. resting alone), and between sexes. Clear barks were given mostly as single calls by immature individuals and adult females in noninteractive contexts. Kwano baboons uttered these calls at a higher rate than Gamgam baboons. Loud calls, consisting of bouts of wahoos, were given by one male at a time, mostly after chasing a group member or without obvious stimulus. These results are now discussed in the light of findings in other baboon populations and taxa.

\subsubsection{Grunts}

Studies in chacma baboons (e.g., Owren et al. 1997; Rendall et al. 1999), hamadryas baboons (Pfefferle \& Fischer 2006), Guinea baboons (Byrne 1981), and olive baboons (Hall \& de Vore 1965; Rowell 1966; Ransom 1981) mentioned contexts of grunts similar to findings of the present chapter. Despite the fact that interactive activities were only a limited fraction of the general activities, many grunts were uttered in these situations in which animals were close to one another (in comparison to non-interactive activities; see results). Grunts are also reported to be often given as a "group contact vocalisation" when the troop is spread out while foraging or resting in sleeping trees, or before a group movement (Ransom 1981; Rendall et al. 1999; K. Meise \& C. Keller, pers. comm.). In social interactions, grunts are uttered during infant handling and by individuals approaching other ones, especially mothers with infants (Ransom 1981; Rendall et al. 1999), in accordance with the present findings. In such contexts, grunts might mollify the recipient, when a male or a dominant female approaches a female or a subordinate one, respectively (Cheney et al. 1995; Silk et al. 1996; Palombit et al. 1999). The proportion of grunts during grooming was low in comparison 
to the proportion of grunts emitted during friendly approaches. This suggests that grunts are used mainly to engage in social interactions, while maintaining the interaction requires fewer of these signals, as suggested by Gilmore (1983a). The context of infant handling may be particular as the handler may have to constantly reassure the mother about the friendliness of its behaviour. The higher proportion of grunts given during infant handling in Kwano troop can be explained by a higher number of black infants in this troop (up to 3 at a time) than in Gamgam troop (one at a time) during the observation period. Grunts have also been associated with reconciliation (Silk et al. 1996; Cheney \& Seyfarth 1997) and general enhancement of friendly social interactions (Cheney et al. 1995; Palombit et al. 1999), but this function was not investigated in this chapter.

Concerning grunt rate, comparison with data from a troop of 18 - 19 olive baboons ranging in Budongo Forest Reserve in Uganda (study site described in Chapter 4) reveals some striking differences. The mean $( \pm \mathrm{SD})$ individual grunt rate was $0.48 \pm 0.20$ grunts $/ \mathrm{min}$ (calculated from 3 adult males $[0.34 \pm 0.08$ grunts $/ \mathrm{min}]$ and 7 adult females $[0.54 \pm 0.20$ grunts / min]; extended analysis from Rahn 2008). This is almost twice as high as grunt rates in the Nigerian troops. For the moment, this difference between the two populations cannot be explained. There may be variations between the Nigerian and Ugandan populations in, for example, the distribution of time between non-interactive and interactive activities, the proportion of grunts given in non-interactive and interactive activities, and the spread of group members. However, these hypotheses require further comparative analyses with equivalent and precise delineations of activity categories. In comparison, chacma baboons from a troop of 30 individuals in Namibia uttered (mean $\pm \mathrm{SD}$ ) $0.35 \pm 0.19$ grunts / min (calculated from 3 adult males [0.16 \pm 0.12 grunts / min] and 9 adult females $[0.42 \pm 0.16$ grunts / $\mathrm{min}]$; C. Keller, pers. comm.). In contrast, adult female chacma baboons from a troop of approximately 70 individuals in Moremi Game Reserve in Botswana grunted at an even lower rate (mean of 22 adult females: 0.11 grunts / min [range: 0.04 0.24 grunts / $\mathrm{min}]$; C. Crockford \& R. Wittig, pers. comm.) than baboons from the Nigerian troops (mean $[ \pm \mathrm{SD}]$ of 15 females: $0.22 \pm 0.11$ grunts $/ \mathrm{min}$ ). C. Crockford and R. Wittig suggested that, as the data were collected during a period of relative social stability, baboons did not have to mollify their conspecifics much before interacting, which would explain the low call rate. However, both Nigerian troops were also relatively stable during the observation periods (pers. obs.), which renders this explaination for inter-taxon difference unlikely. Group size does also not seem to explain inter-taxon differences. In a larger group, a higher interaction rate is expected to lead to a higher grunt rate. However, the reversed tendency in 
grunt rate occurred between the small troops of Nigerian olive baboons and the large troop of chacma baboons from Botswana. This suggests that interaction rate might be dependent on other factors such as food availability or group cohesion. Considering these findings, it appears that inter-population as well as inter-taxon comparisons are hampered if information on some potentially influential factor is missing. Call rates should therefore be carefully calculated in conditions as similar as possible between troops, to allow for further comparisons.

Acoustic variables of grunts in the Nigerian troops are similar to those found in the Ugandan troop of olive baboons (Table 1.4 and Figure 1.2a; see also Chapter 4 for a test of the effect of the population on acoustic structure of adult female grunts). Comparison with the more distantly related chacma baboon taxon, however, reveals some differences. Grunts of adult female chacma baboons have a shorter duration and a higher fundamental frequency than those of adult female olive baboons (Table 1.4 and Figure 1.2a). This is somewhat surprising since chacma baboons are on average slightly heavier than olive baboons (adult chacma baboons: males: $28.8 \pm 2.3 \mathrm{~kg}$; females: $13.9 \pm 1.0 \mathrm{~kg}-16.0 \pm 1.6 \mathrm{~kg}$; Bulger \& Hamilton 1987; adult olive baboons: males: $25.1-27.4 \mathrm{~kg}$; females: $13.3-15.6 \mathrm{~kg}$; Strum 1991; Smith \& Jungers 1997). According to the mechanisms of sound production (Fitch \& Hauser 1995), heavier or larger animals with larger lungs, thicker vocal folds and a longer vocal tract should utter calls with longer duration, lower fundamental frequency and energy concentrated in lower frequencies (reviewed in Ey et al. 2007a; see also Pfefferle \& Fischer 2006). This deviation from expectation might be explained by more subtle anatomical differences. Indeed, rib cage, skull and neck seem to be broader in olive baboons than in chacma baboons which look more slender (pers. obs.). Detailed measures of skull size and neck and rib cage circumferences would be needed to evaluate this impression statistically. Differences in duration, fundamental frequency and peak frequency between sexes in olive baboons match predictions from the mechanisms of sound production cited above, since lighter (and smaller) females have shorter grunts, with higher fundamental frequency and higher peak frequency than heavier (and larger) males. 
a.

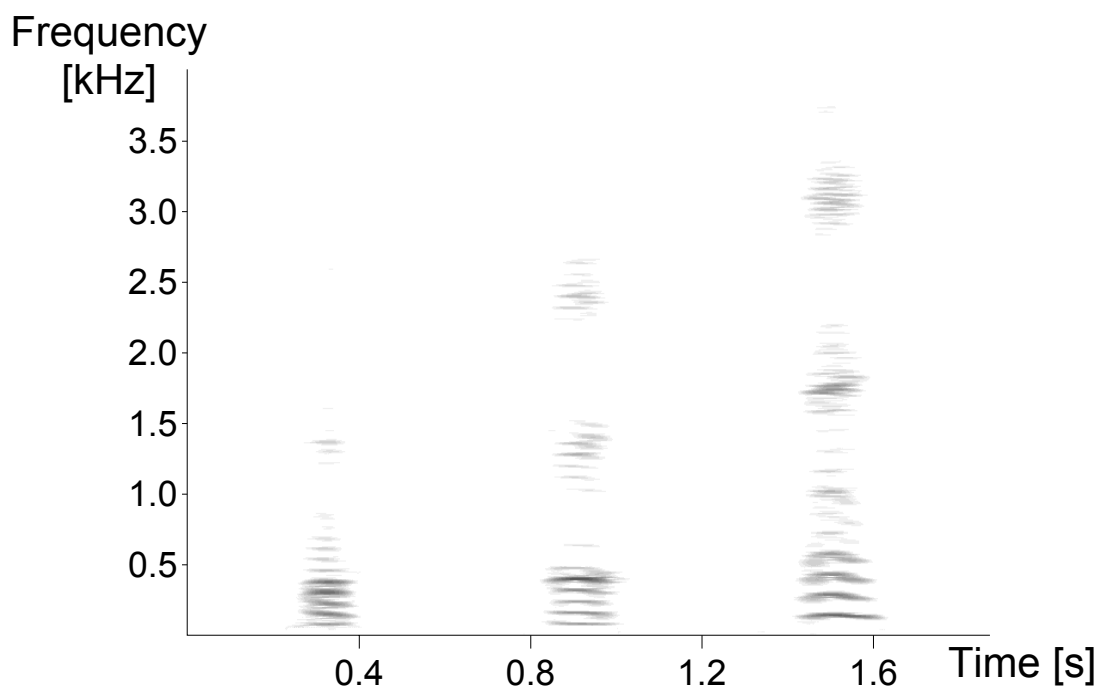

b.

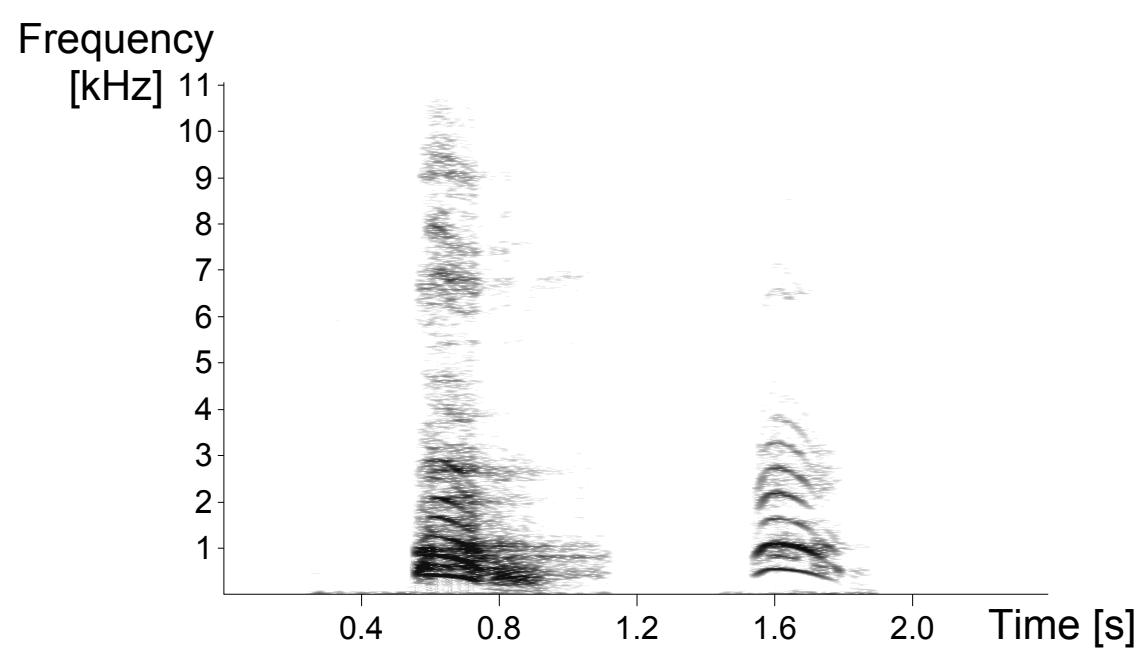

c. Frequency

$[\mathrm{kHz}]$

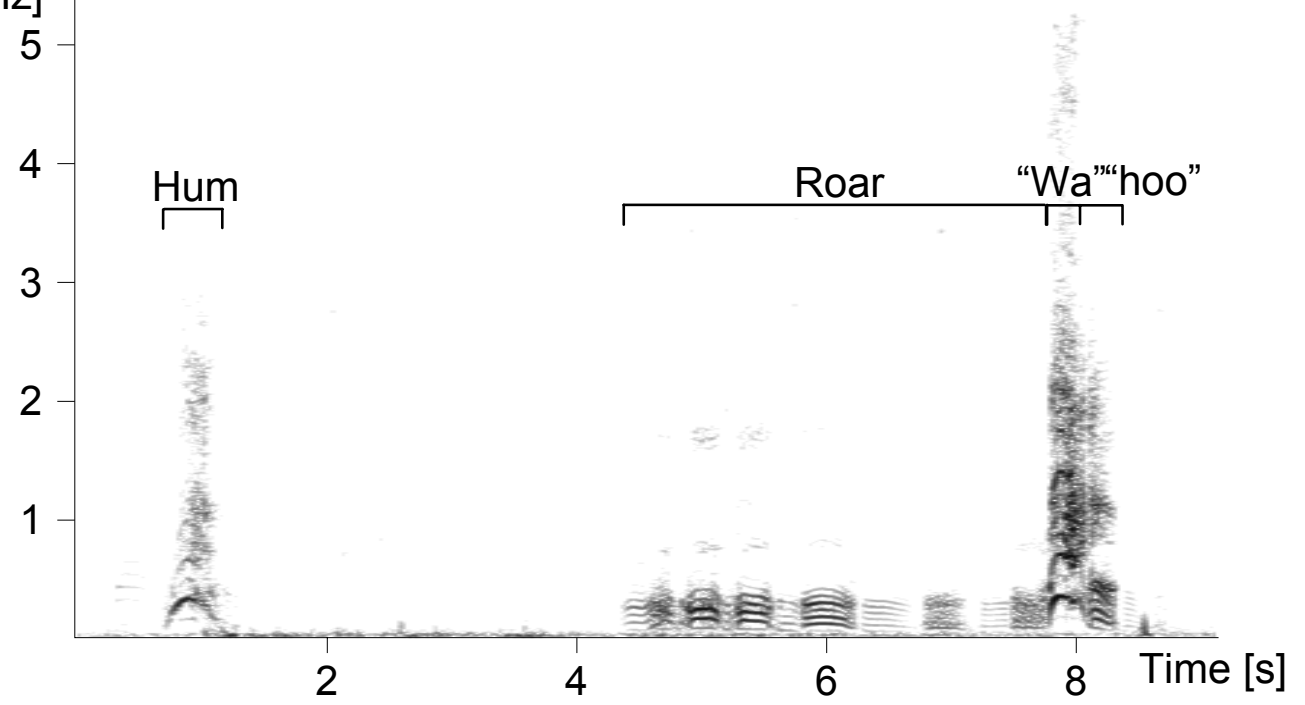

Figure 1.2: Spectrograms of contact vocalisations from different baboon populations and taxa. (a) Grunts of adult females (left: olive baboon from Nigeria; centre: olive baboon from Uganda; right: chacma baboon from Botswana). (b) Clear barks of adult females (left: olive baboon from Nigeria; right: chacma baboon from Botswana). (c) Beginning of a bout of loud call from an adult male chacma baboon from Botswana (compare with Figure 1.1c). 
The unexpected lack of difference in acoustic structure between grunts given in interactive and non-interactive activities appeared to be due to variation in the delineation of contexts. When acoustic variables were compared between more specific contexts (i.e., infant handling vs. resting alone), grunts were found to be longer in the infant handling context than in the context of resting alone. This finding replicated partly results of a study in chacma baboons in Namibia, which also reported variations in other acoustic variables (Meise 2008). In a group of chacma baboons in Botswana, grunts in the infant handling context were found to have a higher second formant frequency and a steeper spectral slope than grunts uttered before a group movement (Owren et al. 1997). These acoustic differences appeared to be salient to the animals, independently from the situation (Rendall et al. 1999). Although these particular variables were not analysed here, results suggest that there are at least some similarities between baboon taxa concerning intra-individual context-related differences in grunt acoustic structure.

\subsubsection{Clear barks}

Clear barks are described for chacma baboons (e.g., Cheney et al. 1996; Fischer et al. 2001a, 2002), Guinea baboons (Byrne 1981), and olive baboons (Hall \& de Vore 1965; Rowell 1966; Ransom 1981). Contrary to previous reports on occurrences of most clear barks in bouts (within-bout rate in olive baboons: 1 - 20 barks / min; Ransom 1981), the present chapter describes them mostly as single calls. Adult females and juveniles of both troops accounted for the majority of barks, as in chacma baboons in Botswana (Cheney et al. 1996). Contexts of utterances described in the present chapter were similar to those cited in the literature for both olive and chacma baboons, that is, separation from the rest of the troop (but not necessarily complete isolation) or from particular individuals (Ransom 1981; Cheney et al. 1996; Rendall et al. 2000; Fischer et al. 2001a). According to previous studies, baboons seem to call mostly according to their own spatial position in the group, which might also be linked to their rank (Cheney et al. 1996; Rendall et al. 2000).

Both Nigerian troops presented a very low group bark rate, especially in Gamgam troop. It was not rare to spend entire days without hearing any single clear bark (Gamgam: 32 of 57 observation days; Kwano: 13 of 66 observation days). A slightly higher group bark rate was found in the Ugandan troop of $18-19$ olive baboons ( 2.88 barks $/ \mathrm{h}$, that is, 0.15 barks / h / individual; Rahn 2008), but it was also not uncommon to spend entire days without hearing any barks (C. Rahn, pers. comm.). Guinea baboons in Senegal were found to 
have a high average bark rate (19.7 barks / h for a group of 150 - 200 animals [calculated from call rates in 5 min intervals]; Byrne 1981) but their bark rate corrected for group size (approximately 0.11 barks / h / individual) was similar to the low bark rate in Nigerian olive baboons. In contrast, bark rates in the troop of around 70 chacma baboons in Botswana were much higher than those calculated in olive baboons in Nigeria and Uganda. Adult females as well as juveniles and infants older than 6 months uttered on average 1.1 barks / h / individual, and subadult and adult males 0.2 barks / h / individual (Cheney et al. 1996). These rates were calculated for the $2.5 \mathrm{~h}$ after baboons left their sleeping site. A retrospective calculation over the same daytime period still revealed much lower rates for Nigeria (unpubl. data). More data on group movement patterns and inter-individual distances are needed to evaluate the contribution of these factors to usage of clear calls to explain such variations.

The much lower bark rate in Gamgam troop cannot be explained by a lower group spread, as individuals did not tend to stay closer to one another than in Kwano troop. Lower bark rates in Gamgam troop could be due to a reduced usage of vocal signals as a mean to maintain contact. Indeed, this troop ranged mostly in woodland savannah where visual contact can be maintained over large distances (when the grass is low). In contrast, forest covers most of the Kwano troop range. However, this explanation is unlikely because, contrary to expectations, no variation in bark rate between forest and woodland savannah was found (see Chapter 5). Another explanation would involve social factors. For instance, Kwano troop might undergo more fission-fusion than Gamgam troop, and therefore animals in subgroups might have more occasions to utter clear barks to maintain or recover contact with the rest of the group (Byrne 1981; Rendall et al. 2000). To test this hypothesis, future studies should monitor the actual group size to provide an estimation of the frequency at which troops split and rejoin while recording bark rates. This measure would be quite helpful in comparative studies. Besides, bark rates might also be strongly dependent on the immediate situation (separation from the group) and the internal state (arousal due to isolation) of the caller (Cheney et al. 1996; Rendall et al. 2000).

When acoustic features of clear barks from adult female olive baboons in Nigeria are compared to those from adult female chacma baboons in Botswana (extended analyses from Fischer et al. 2001a and Ey et al. 2007b), calls of olive baboons appeared to be longer than those of chacma baboons (Table 1.6). This might be due to the fact that the "hoo" part was always present in clear barks of Nigerian olive baboons but not always in those of chacma baboons (Figure 1.2b). The fundamental frequency did not differ much between chacma and olive baboons. This does not corroborate what was found for grunt fundamental frequency, 
but one has to consider that the fundamental frequency of barks is not the minimum fundamental frequency of the animal, which should theoretically be most strongly constrained by body size (Fitch \& Hauser 1995). Finally, energy was concentrated in lower frequencies in clear barks of Nigerian olive baboons than in those of chacma baboons (Table 1.6). This difference might stem from slight anatomical differences, as hypothesised for grunts, and would require further investigations.

\subsubsection{Loud calls}

The present findings revealed some differences in the contexts in which loud calls were uttered between olive and chacma baboons. The most obvious one is that, in Nigerian olive baboons, there was no alternation between callers. In contrast, in contest between male chacma baboons, callers often alternate (this characteristic was used to test whether baboons were able to distinguish dominance ranks in vocal interactions in playback experiments; Kitchen et al. 2005). In Nigerian olive baboons, loud calls were rarely (if at all during observation time) given after calls from males of the same troop or of other troops (this situation would parallel contest between males in chacma baboons). This seems to be the case also in other troops of olive baboons (e.g., in Laikipia District, Kenya: A. Lemasson, pers. comm.). However, in the present chapter, the context of calling could be clearly determined in only few cases. Therefore, more data on loud call occurrences should be collected in other olive baboon populations before drawing any conclusions. These additional data should allow investigations on potential differences between baboon taxa in the function of male loud calls suggested by the present results.

The rate at which bouts of loud calls were given did not differ much between Nigerian olive baboons ( $0.14-0.16$ bouts / h) and chacma baboons in Botswana (once every $11.3 \mathrm{~h}$ of observation, which translates into 0.09 bouts $/ \mathrm{h}$ ). This is intriguing because the troop in Botswana contained much more males (12 adult males on average in a group of $78-88$ individuals; Kitchen et al. 2003a). The high bout rate (related to the number of males) in Nigerian olive baboons might stem from the fact that loud call bouts are often uttered after agonistic interactions with a female or a juvenile of the troop. This situation occurs relatively often but was not cited as a context for loud calls in chacma baboons. This strengthens the impression of an inter-taxon difference in the function of male loud calls. In the present chapter, the within-bout bark rate was similar when calculated over the total bout duration or over inter-bark intervals. In contrast to the emission rate of bouts however, this mean $( \pm$ SD) 
within-bout bark rate was much lower in the olive baboons under study $(8.85 \pm 5.43$ barks $/ \mathrm{min})$ than in chacma baboons in Botswana $(30.5 \pm 4.0 \mathrm{barks} / \mathrm{min}$; Fischer et al. 2002). This might be due to the fact that males in both Nigerian troops do not use loud calls in contest situations (despite the entrance of new males in Kwano troop; pers. obs.). In contests, call rate reflects fighting ability and dominance rank of the caller. Higher ranking males (which have potentially the best condition) participate in more bouts, call at higher rates, with more calls within a bout and longer bouts in comparison to low or midranking males (Kitchen et al. 2003b). In other situations which do not involve intra-sexual competition (e.g., after chasing a female like in Nigerian baboons), call rate intuitively does not need to be high since the physical dominance of males over females or juveniles is established. Nevertheless, additional recordings of bouts are still needed to examine whether the within-bout bark rate also depends on the contexts in which bouts are given (even if differing from contest situations) in olive baboons. The rank of the caller, as well as its state of exhaustion also influence loud calls acoustic features in chacma baboons (Fischer et al. 2004), and these changes are salient to other males (Kitchen et al. 2005). Investigating whether and to which degree these factors determine loud calls acoustic features in olive baboons might also help to shed light on the functions of these calls in this taxon. Such a study would require additional good-quality recordings.

Finally, despite potential functional differences between baboon taxa, loud call bouts were acoustically similar between olive baboons (Figure 1.1c) and chacma baboons (Figure 1.2c). Bouts began with a roar or a hum and a roar in both taxa, and then went on with a series of wahoos. Unfortunately, the quality of most recordings was not sufficient to conduct any acoustic analyses and only one male contributed to most of the few good-quality recordings. This did not allow any reliable comparison of wahoo acoustic features between chacma and olive baboons. Additional recordings are needed for finer analyses. These acoustically similar vocalisations having a potentially different function between baboon taxa illustrate a phylogenetically constrained vocal production (only a few call types are available to the animals) and a flexible usage of these few call types (the same call types could be uttered in different contexts) within the genus Papio, as hypothesised.

To conclude, the contexts in which contact calls were given were generally similar between baboon taxa, except for loud calls. As predicted, the acoustic structure of grunts, clear barks and loud calls presented only minor differences between populations or taxa, reflecting some phylogenetic constrain on vocal production. In contrast, variability was 
important in grunt and bark rates between olive baboon populations and between olive and chacma baboons, confirming flexibility in call usage. Various factors such as group dispersal or fission-fusion tendency might interplay to regulate these call rates. Differences in the contexts of utterance and within-bout bark rate for loud calls between olive and chacma baboons could reflect a differential function of these calls between the two taxa. Future studies will need to integrate factors such as the social system and the environment to shed additional light on inter-population or inter-taxon variability. Short-term variations in factors such as environment (Chapter 4) and distances between individuals should also be examined to investigate the degree of flexibility in vocal communication within individuals or groups. Only the consideration of numerous sources of variation in vocal behaviour will help to further unravel the driving forces in the evolution of baboon vocal communication. 


\title{
Chapter 2
}

\section{The "acoustic adaptation hypothesis" - A review of the evidence from birds, anurans and mammals}

\begin{abstract}
Structural and acoustic properties of the environment influence sound propagation. Many studies examined whether various species of anurans, birds and mammals adjust usage and structure of their vocal signals to limit degradation during propagation ("acoustic adaptation hypothesis"). The present chapter examines how widespread such adaptations actually are across taxa. First, evidence for environment-related adjustments in usage of vocal signals is collected from studies in birds and other vertebrates (i.e., anurans and mammals). Second, a meta-analysis conducted by Boncoraglio and Saino (2007) on the influences of the environment on acoustic structure of avian vocalisations is taken as a reference, and results from additional studies in birds are reviewed and compared to its conclusions. Finally, evidence from similar studies conducted in anurans and mammals is collected and discussed. Concerning usage of vocal signals, evidence of environmental adaptations in the few studies found was more widespread in anurans and mammals than in birds. Regarding structure of vocal signals, evidence from additional studies in birds did not clearly confirm results of the meta-analysis of Boncoraglio and Saino (2007). Pooling all bird studies together presented minimum frequency, frequency modulations and frequency range as acoustic variables most often adjusted to the environment, in contrast to temporal features, repetition rate and maximum frequency. The few studies conducted in anurans and mammals did not allow to point out any acoustic variable preferentially showing environment-related variations. Overall, evidence for the acoustic adaptation hypothesis was not as widespread as expected across taxa. The different aspects of vocal behaviour adapted to environmental conditions varied according to the species considered, the local habitat or the function of the signals studied.
\end{abstract}




\subsection{INTRODUCTION}

Vocal signals contain cues about caller's identity, size, sexual status or fighting ability or about external events such as predator type, for instance. This information is transferred between signallers and receivers and might be used in reproductive and survival strategies. If some of this content is degraded during propagation, signals might become less effective and fail to fulfil their functions. The influence of the environment on aerial vocal communication has therefore triggered much attention, especially in taxa in which acoustic communication in the air is widespread (i.e., in various species of insects [which will not be considered anymore hereafter], anurans, birds, and mammals). Experiments of sound propagation clearly demonstrate the effect of the environment on sound acoustic structure (reviewed in Piercy et al. 1977 and Wiley \& Richards 1978). For instance, high-frequency sounds are attenuated more rapidly than low-frequency sounds (e.g., temperate habitats: Marten \& Marler 1977; tropical forest: Marten et al. 1977; rainforest, riverine forest and savannah: Waser \& Brown 1986). However, very low frequencies are considerably attenuated by the ground effect (i.e., long waves of low-frequency sounds are refracted in the ground) when broadcasted from a limited height (temperate habitats: Marten \& Marler 1977; tropical forest: Marten et al. 1977). Transmission is then optimal for frequencies falling in a "ground effect window" (usually between 1 and $3 \mathrm{kHz}$ ), in which attenuation due to the ground effect is reduced (e.g., tropical forest: Morton 1975; mixed deciduous forest: Richards \& Wiley 1980; dry open vs. moist dense forests: Padgham 2004). In addition, each environment has its own acoustic characteristics for sound transmission (e.g., Aylor 1971; Linskens et al. 1976; Date \& Lemon 1993), and its own daily distribution of ambient noise (e.g., Waser \& Brown 1986; Schneider et al. 2008), since vegetation structure, vegetation density, climatic conditions, soil features, and ambient noise (frequency ranges, amplitude) are specific to each environment. For instance, in closed habitats like forests (i.e., with a high vegetation density), surfaces for reverberation and absorption are more important and acoustic conditions are more constant than in open habitats (Waser \& Brown 1986). Open habitats, with less dense vegetation and more turbulent air, provide more variable conditions for sound propagation (Morton 1975), and the visual and auditory communication channels can complement each other (Brown et al. 1995; Brown \& Handford 2000). Therefore, the selection pressure imposed by the environment is assumed to be stronger in closed than in open habitats. Animals are then expected to adjust usage as well as acoustic structure of their vocal signals to optimise propagation ("acoustic adaptation hypothesis": Morton 1975, here extended to usage), and 
more clearly so in closed than in open habitats (Waser \& Brown 1986). This hypothesis entails general predictions.

They concern various aspects of call usage. Vocalisations are expected to be more stereotyped in closed than in open habitats, since the availability of the visual channel is restricted and transmission conditions are stable in closed habitats (Morton 1975; Brown \& Handford 2000). This would facilitate signal detection by recipients. Animals are also expected to adjust the time (e.g., Henwood \& Fabrick 1979) and place of calling (e.g., height: Marten \& Marler 1977; Marten et al. 1977), sensu when and where they vocalise, to the local sound transmission and ambient noise conditions. For instance, calling from a high post might increase propagation distance (Mathevon et al. 1996; Padgham 2004). Finally, animals are expected to call at a higher rate in closed habitats to increase the likelihood of their calls reaching the intended recipient. Indeed, calls given in a closed environment are more likely to be attenuated through vegetation and repeating them would increase the likelihood of them being detected and the accuracy with which call types are recognised. A higher call rate in a closed habitat might also compensate the limited availability of the visual communication channel, in comparison to an open habitat.

Regarding the structure of vocal signals, general predictions concern eight categories of acoustic variables (summarised in Table 2.1). Environment-related variations might affect one or more of them. First, vocalisations are expected to (1) be longer in closed than in open habitats. Lengthening vocal signals might increase the likelihood of detection and might allow to use the amplitude of reverberated sound waves (substantial in closed habitats) to increase propagation distance (Nemeth et al. 2006). In parallel, shorter calls might be less susceptible to the fluctuating transmission conditions in open habitats (e.g., Morton 1975). Second, vocalisations are expected to present (2) a lower repetition rate to reduce overlap with reverberated waves and a lower frequency-modulation rate in closed than in open habitats. Propagation distance and transmission quality of slow frequency-modulations are generally better than that of rapid ones (Brown \& Handford 2000; Naguib 2003), while transmission quality of rapid frequency modulations is more consistent in open than in closed habitats (Richards \& Wiley 1980; Brown \& Handford 2000). Third, vocalisations should have (3) a more limited proportion of frequency-modulations in closed than in open habitats since transmission of frequency-modulated elements is not as consistent in closed habitats as in open ones (Wiley \& Richards 1978; Richards \& Wiley 1980). Fourth, vocalisations should have lower (4) maximum, (5) minimum and (6) mean frequencies and (7) energy concentrated in lower frequencies (lower dominant or peak frequency [i.e., frequency with the highest 
amplitude]) in closed than in open habitats, since low frequencies transmit further than high frequencies, this difference being larger in closed than in open habitats (Chapuis 1971; Morton 1975; Marten \& Marler 1977; Marten et al. 1977). Finally, vocalisations are expected to (8) have a narrower frequency range in closed habitats than in open habitats because acoustic conditions are more constant in the former (Morton 1975; Shy \& Morton 1986). It would permit to concentrate energy in frequencies which undergo limited attenuation (Wiley \& Richards 1978).

Table 2.1: General predictions concerning adjustments to the environment in acoustic structure of vocal signals.

\begin{tabular}{l|ll} 
In a closed habitat & longer duration \\
(2) & lower repetition rate \\
(3) & fewer frequency-modulated elements \\
(5) & lower minimum frequency \\
(6) & lower mean frequency \\
(7) & lower dominant frequency \\
(8) & narrower frequency range
\end{tabular} \mid than in an open habitat

Several studies in birds (Carolina wrens [Thryothorus ludovicianus]: Gish \& Morton 1981; song sparrows [Melospiza melodia]: Shy \& Morton 1986; 3 species of nightingales: Sorjonen 1986a), but also in other vertebrates (frogs [Ranidella riparia and $R$. signifera]: Odendaal et al. 1986; a forest subspecies of cricket frog [Acris c. crepitans]: Ryan et al. 1990; forest vs. savannah monkey species: Brown et al. 1995; Japanese macaques: Sugiura et al. 2006) actually found that vocal signals propagate better in habitats where callers use to live than in other habitats. This suggests that vocal signals of these species are well adapted to the acoustic conditions of their actual environment. However, a non-negligible number of studies in birds (American redstart [Setophaga ruticilla]: Date \& Lemon 1993; 5 species of wood warblers: Fotheringham et al. 1997; blue tits [Parus caeruleus]: Doutrelant \& Lambrechts 2001; south African passerine species: Saunders \& Slotow 2004), and in other vertebrates (4 marmot species: Daniel \& Blumstein 1998; 5 frog species: Penna \& Solis 1998; 22 frog species: Kime et al. 2000) failed to find this trend. Such ambiguous findings raise the following questions: do variations in usage as well as structure of vocal signals actually reflect some adaptations to environmental conditions and how widespread are such adaptations across taxa?

The present chapter investigates these questions by comparing results of previous studies in birds as well as in anurans and mammals to the above-cited general predictions. It 
first examines whether usage of vocal signals is adjusted to environmental conditions in birds and in other vertebrates (i.e., anurans and mammals), and compares the evidence between the two groups. Secondly, this chapter examines whether the acoustic structure of avian vocalisations varies with the environment according to general predictions. A meta-analysis controlling for phylogeny and body size has already been conducted on this subject by Boncoraglio and Saino (2007). Results from a set of additional studies not included in this meta-analysis are then reviewed and findings are compared with those of the meta-analysis (lower minimum and dominant frequencies, and narrower frequency range in closed habitats than in open habitats, and no effect on temporal features). Finally, this chapter reviews the evidence for environment-related variations in acoustic structure of vocal signals in anurans and mammals, as a parallel to the investigations conducted in birds. Trends in line with general predictions are expected to emerge from findings in a majority of studies across all taxa. Besides, results found by Boncoraglio and Saino (2007) should be strengthened by the additional studies in birds not included in this meta-analysis. Factors potentially explaining the tendencies found in this review are then discussed.

\subsection{SELECTION OF STUDIES AND ORGANISATION OF THE SYNTHESIS}

The available literature was scanned using the Web of Knowledge database, with combinations of the following keywords: "habitat", "environment", "adaptation", "vocalisation", "song", "call" and "vocal communication". Studies in birds, anurans and mammals were selected, while those in invertebrates and other vertebrates were deliberately ignored. The focus was set on vocal communication in air. The influence of ambient noise is evoked only briefly for call usage. For a complete review on acoustic communication in noise, see Brumm \& Slabbekoorn (2005). The influence of the habitat is examined separately on usage and on acoustic variables. Concerning usage, more details about the studies considered (birds: 11 studies; anurans and mammals: 11 studies) are provided in Appendix 1.

Acoustic variables are grouped into eight categories corresponding to general predictions: temporal parameters (i.e., duration of calls, of units or of intervals between units), repetition rate (e.g., frequency-modulation or trill rate), frequency modulations (i.e., presence or absence, proportion), maximum frequency, minimum frequency, mean frequency, dominant frequency, and frequency range (i.e., maximum minus minimum frequency). Table 2.2 summarises the evidence for each acoustic variable category in birds. Numbers in this table are referring to the list of all collected studies on the influence of the environment 
on acoustic structure in birds in Appendix 2. Bold numbers refer to studies included in the meta-analysis of Boncoraglio and Saino (2007). Table 2.3 presents studies investigating the same question in anurans and mammals.

Phylogenetic relationships (e.g., McCracken \& Sheldon 1997) and body size (reviewed for primates in Ey et al. 2007a) can influence acoustic features of vocalisations. Distinction is thus made between studies controlling for phylogenetic relationships (between species or between populations), those controlling for the influence of body size, those controlling for both factors and those controlling for none of these two factors. If a study includes analyses with different control levels (phylogeny, body size, both factors or none), results from conditions controlling the maximum number of factors are cited. Studies comparing populations or groups of the same species (intra-species) are distinguished from those comparing different species (inter-species). Studies conducted in birds (42 studies, among which 22 were included in the meta-analysis of Boncoraglio and Saino [2007]) and those conducted in anurans and mammals (12 studies) are considered separately. Special attention is given to the few studies conducted in anurans and mammals, since these taxa are less investigated than birds. Findings from studies in birds are then more condensed.

\subsection{ENVIRONMENTAL INFLUENCES ON USAGE OF VOCAL SIGNALS}

\subsubsection{In birds (Appendix 1a)}

Only few studies consider avian vocal repertoires in general. Birds are expected to use frequencies clearly different from ambient noise, but this is not the case in songs of Western gerygones (Gerygone fusca fusca: Baker 2006), red-capped robins (Petroica goodenovii: Baker 2006) and great tits (Parus major: Slabbekoorn \& Peet 2003; Slabbekoorn \& den BoerVisser 2006). No other study examining this aspect has been found. The time of calling or singing can also be adjusted to ambient noise and transmission conditions, as in nightingales (Luscinia megarhynchos: Brumm 2006). In contrast, blackcaps (Sylvia atricapilla) do not sing at times when background noise and transmission conditions are optimal (Dabelsteen \& Mathevon 2002). Furthermore, individuals are expected to use signalling locations which optimise transmission of their vocalisations, as it has been demonstrated in sedge warblers (Acrocephalus schoenobaenus) and great reed warblers (A. arundinaceus: Jilka \& Leisler 1974), in great tits (Hunter 1980), partially in black birds (Turdus merula: Dabelsteen et al. 
1993), in wrens (Troglodytes troglodytes: Mathevon et al. 1996), and in five sympatric species of antbirds (Nemeth et al. 2001). However, this is not the case in reed warblers (Acrocephalus scirpaceus: Jilka \& Leisler 1974). Steer's liocichla (Liocichla steerii) often sing in duets in closed habitat, probably to exchange information about their location between pair members (Mays et al. 2006). No study investigating variations of call rate with environmental features other than ambient noise has been found in birds.

\subsubsection{In mammals and anurans (Appendix 1b)}

A few trends concerning the vocal repertoire in general emerge. Primate species living in closed habitats seem to have discrete call types, whereas those living in more open habitats have a more graded vocal repertoire (Waser \& Brown 1986). In addition, calls emitted in closed habitats seem to be less variable and therefore more stereotyped than those given in open habitats (various primate species: Marler 1974, Waser \& Waser 1977, Waser 1982; chimpanzees: Mitani et al. 1999). Individuals are also expected to use frequencies clearly different from ambient noise. This has been confirmed in calls of pygmy marmosets (de la Torre \& Snowdon 2002) and in loud calls of Siberut primates (Kloss gibbons [Hylobates klossii], Mentawai macaques [Macaca siberu], Mentawai leaf monkeys [Presbytis potenziani], pig-tailed langurs [Simias concolor]: Schneider et al. 2008). Furthermore, some primate species (e.g., black and white colobus [Colobus guereza]: Waser \& Waser 1977; cotton-top tamarins: Egnor et al. 2007; Kloss gibbons, Mentawai macaques, Mentawai leaf monkeys, pig-tailed langurs: Schneider et al. 2008) time their calls according to ambient noise and transmission conditions. Animals should use signalling locations which optimise sound transmission (model in Northern spring peepers [Pseudacris c. crucifer]: Parris 2002). However, such spatial adjustment could be explained by other social or ecological factors in Siberut primates (Schneider et al. 2008; see discussion). Finally, some primate species (greycheeked mangabeys [Cercocebus albigena] vs. baboons [Papio cynocephalus]: Waser, unpubl. data in Waser \& Waser 1977; chacma baboons: Rendall et al. 2000; Japanese macaques: Koda et al. 2008) call at a higher rate in closed habitats than in more open habitats, as expected. 


\subsection{ENVIRONMENTAL INFLUENCES ON ACOUSTIC STRUCTURE OF VOCAL SIGNALS}

\subsubsection{In birds (Table 2.2, additional studies)}

Overall, bird vocalisations tend to have a lower maximum frequency in closed than in open habitats, while repetition rate varies independently from the environment according to findings in the additional studies (Table 2.2). Variations in other acoustic variables remain ambiguous. These results are detailed hereafter.

Evidence for environmental influences on temporal features is limited: results in 1 of 3 studies without any control on phylogeny and body size and in 3 of 6 studies controlling for phylogeny and/or body size are in accordance with general prediction (1) (Table 2.2a). Results in 4 of 5 studies without any control and in 4 of 6 studies controlling for phylogeny and / or body size are not in line with general prediction (2) of a lower repetition rate in closed than in open habitats (Table 2.2b). The sample of studies examining frequency modulations is small (5 studies; Table 2.2c). Therefore, no obvious trend can be discussed for this acoustic variable, but 3 of 5 studies present results in line with prediction (3), with fewer frequency modulations in closed than in open habitats. Results in none of 2 studies without any control but in 5 of 6 studies controlling for phylogeny and / or body size find results in accordance with general prediction (4), with a lower maximum frequency in closed than in open habitats (Table 2.2d). Results in none of 2 studies without any control but in 3 of 5 studies controlling for phylogeny and/or body size are in line with general prediction (5), with a lower minimum frequency in closed than in open habitats (Table 2.2e). Only 4 studies examine variations of mean frequency with environmental features. Results of 2 of them are in accordance with general prediction (6), with a low mean frequency in closed habitats, while those of the 2 others are not (Table 2.2f). Conclusions are difficult to draw from this small sample. Results in one of 2 studies without any control and in 2 of 3 studies controlling for phylogeny and / or body size are in line with general prediction (7), with a lower dominant frequency in closed than in open habitats (Table $2.2 \mathrm{~g}$ ). Finally, both studies without any control but only 3 of 7 studies controlling for phylogeny and / or body size confirm general prediction (8), with a narrower frequency range in closed than in open habitats (Table 2.2h). The tendency is then not clear for this variable but it goes in the direction predicted. 
Table 2.2: Studies investigating the influence of the habitat on (a) temporal parameters, (b) repetition rate, (c) frequency modulations, (d) maximum frequency, (e) minimum frequency, (f) mean frequency, (g) dominant frequency and (h) frequency range. Numbers refer to the studies listed in Appendix 2. Bold numbers refer to studies included in the meta-analysis of Boncoraglio and Saino (2007). "Additional studies" are the ones not included in this meta-analysis. The first line indicates the level of control: control of phylogeny but not of body size, control of body size but not of phylogeny, control of phylogeny and body size, and no control of these factors. "yes": the habitat has an influence in accordance with the general predictions summarised in Table 2.1. "no": no influence of the habitat was found. "inv": an influence of the habitat was found but the effect was contrary to that predicted (Table 2.1). Cells in grey indicate that no study was found for this category.

\begin{tabular}{|c|c|c|c|c|c|c|c|c|c|c|c|c|}
\hline \multirow{2}{*}{ a. Temporal parameters } & \multicolumn{3}{|c|}{ Phylogeny (no body size) } & \multicolumn{3}{|c|}{ Body size (no phylogeny) } & \multicolumn{3}{|c|}{ Phylogeny + Body size } & \multicolumn{3}{|c|}{ No control } \\
\hline & inv & no & yes & inv & no & yes & inv & no & yes & inv & no & yes \\
\hline Inter-species & 33 & 26 & 36 & & 16 & 2 & & 5,27 & & & 31 & 3 \\
\hline Intra-species & & 15 & 17 & & & 11,28 & & 21 & & & $\begin{array}{c}1, \mathbf{1 3}, 30, \\
42\end{array}$ & \\
\hline Additional studies & 1 & 0 & 1 & & 1 & 2 & & 1 & & & 2 & 1 \\
\hline Total number of studies & 1 & 2 & 2 & & 1 & 3 & & 3 & & & 5 & 1 \\
\hline
\end{tabular}

b. Repetition rate

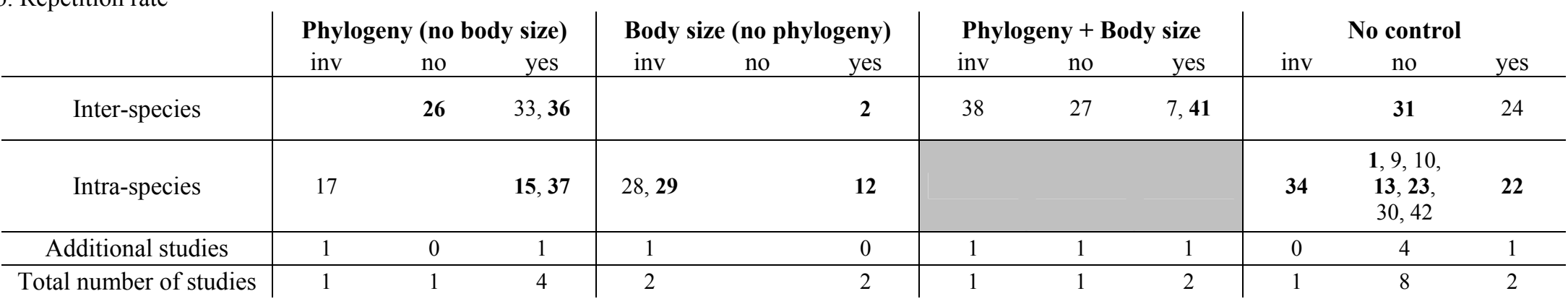




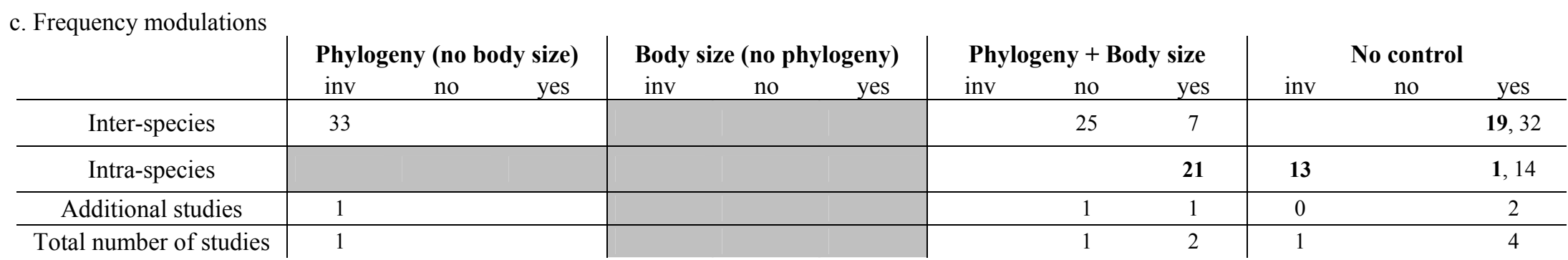

d. Maximum frequency

\begin{tabular}{|c|c|c|c|c|c|c|c|c|c|c|c|}
\hline & \multicolumn{2}{|c|}{ Phylogeny (no body size) } & \multicolumn{3}{|c|}{ Body size (no phylogeny) } & \multicolumn{3}{|c|}{ Phylogeny + Body size } & \multicolumn{3}{|c|}{ No control } \\
\hline & inv & yes & inv & no & yes & inv & no & yes & inv & no & yes \\
\hline Inter-species & & 36 & 2 & 8 & 16 & 35 & 5 & $4,38,41$ & & & \\
\hline Intra-species & 15,37 & 17 & 11,29 & & 12,28 & & & & 22 & $\begin{array}{c}\mathbf{1 3}, \mathbf{2 3} \\
30,42\end{array}$ & \\
\hline Additional studies & 0 & 2 & 1 & 0 & 2 & 0 & 0 & 1 & 0 & 2 & \\
\hline Total number of studies & 2 & 2 & 3 & 1 & 3 & 1 & 1 & 3 & 1 & 4 & \\
\hline
\end{tabular}

e. Minimum frequency

\begin{tabular}{|c|c|c|c|c|c|c|c|c|c|c|c|c|}
\hline \multirow{2}{*}{ 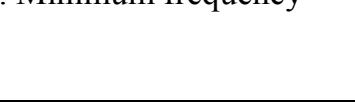 } & \multicolumn{3}{|c|}{ Phylogeny (no body size) } & \multicolumn{3}{|c|}{ Body size (no phylogeny) } & \multicolumn{3}{|c|}{ Phylogeny + Body size } & \multicolumn{3}{|c|}{ No control } \\
\hline & inv & no & yes & inv & no & yes & inv & no & yes & inv & no & yes \\
\hline Inter-species & & 36 & & & & 8 & & 35 & 4,38 & & & \\
\hline Intra-species & & & $\begin{array}{c}15,17, \\
37 \\
\end{array}$ & 11 & 12 & 28,29 & & & 21 & 34 & $\mathbf{1 3}, 30,4$ & \\
\hline Additional studies & & 1 & 1 & 1 & 0 & 1 & & 0 & 1 & 0 & 2 & \\
\hline Total number of studies & & 1 & 3 & 1 & 1 & 3 & & 1 & 3 & 1 & 3 & \\
\hline
\end{tabular}




\begin{tabular}{|c|c|c|c|c|c|c|}
\hline \multirow[t]{2}{*}{ f. Mean frequency } & Phylogeny (no body size) & Body size (no phylogeny) & Phylogeny + Body size & \multicolumn{3}{|c|}{ No control } \\
\hline & inv no yes & yes & inv no & inv & no & yes \\
\hline Inter-species & 18,26 & & 7,27 & & & \\
\hline Intra-species & & & & & 20 & 39,40 \\
\hline Additional studies & 1 & & 2 & & 1 & 0 \\
\hline Total number of studies & 2 & & 2 & & 1 & 2 \\
\hline
\end{tabular}

g. Dominant frequency

\begin{tabular}{|c|c|c|c|c|c|c|c|c|c|c|c|}
\hline \multirow{2}{*}{-5} & \multicolumn{2}{|c|}{ Phylogeny (no body size) } & \multicolumn{3}{|c|}{ Body size (no phylogeny) } & \multicolumn{3}{|c|}{ Phylogeny + Body size } & \multicolumn{3}{|c|}{ No control } \\
\hline & inv & yes & inv & no & yes & inv & no & yes & inv & no & yes \\
\hline Inter-species & & 18 & & 2 & 8 & 35 & 5,41 & 4,25 & & & 19,31 \\
\hline Intra-species & 17 & 15 & & 29 & & & & 21 & & $\mathbf{1}, \mathbf{1 3}, 42$ & 6 \\
\hline Additional studies & 1 & 1 & & 0 & 0 & 0 & 0 & 1 & & 1 & 1 \\
\hline Total number of studies & 1 & 2 & & 2 & 1 & 1 & 2 & 3 & & 3 & 3 \\
\hline
\end{tabular}

h. Frequency range

\begin{tabular}{|c|c|c|c|c|c|c|c|c|c|c|c|c|}
\hline & \multicolumn{3}{|c|}{ Phylogeny (no body size) } & \multicolumn{3}{|c|}{ Body size (no phylogeny) } & \multicolumn{3}{|c|}{ Phylogeny + Body size } & \multicolumn{3}{|c|}{ No control } \\
\hline & inv & no & yes & inv & no & yes & inv & no & yes & inv & no & yes \\
\hline Inter-species & 33 & 26 & 18,36 & & 16 & & 35 & 5 & 4,7 & & 31 & 3,19 \\
\hline Intra-species & & 17 & & 11 & & 12,28 & & & & & & $\mathbf{1}, 6,34$ \\
\hline Additional studies & 1 & 1 & 1 & 1 & 1 & 1 & 0 & 0 & 1 & & 0 & 2 \\
\hline Total number of studies & 1 & 2 & 2 & 1 & 1 & 2 & 1 & 1 & 2 & & 1 & 5 \\
\hline
\end{tabular}




\subsubsection{In anurans and mammals (Table 2.3)}

Concerning temporal features, the only clear evidence in accordance with general prediction (1) (i.e., calls or elements longer in a closed environment than in an open one) occurs between two populations of chimpanzees in introductory and build-up elements of pant hoots (Mitani et al. 1999). In contrast, several studies find results contrary to general prediction (1) (Ranidella signifera vs. Ranidella riparia calls: Odendaal et al. 1986; Galago garnettii vs. Galago crassicaudatus loud calls: Masters 1991; echolocation calls of 4 Pipistrellus species: Kalko \& Schnitzler 1993; echolocation calls of 6 species of emballonurid bats: Kalko 1995; black myotis [Myotis nigricans] echolocation calls: Siemers et al. 2001; little brown bat [Myotis lucifigus] echolocation calls: Wund 2005). Two other studies do not find any relationship between habitat characteristics and temporal features (Gunnison's prairie dog [Cynomys gunnisoni] alarm calls: Perla \& Slobodchikoff 2002; advertisement calls of 95 Bolivian frog species: Bosch \& de la Riva 2004).

In accordance with general prediction (2), two studies highlight a lower repetition rate in closed than in open habitats (Ranidella signifera vs. Ranidella riparia calls: Odendaal et al. 1986; build-up elements of pant hoots between two chimpanzee populations: Mitani et al. 1999). In contrast, the opposite relationship occurs between loud calls of two species of bushbabies (Galago garnettii vs. Galago crassicaudatus: Masters 1991). No relationship between repetition rate and environmental features appears in calls of 56 central Amazon frog species (Zimmerman 1983) and in advertisement calls of 95 Bolivian anurans (Bosch \& de la Riva 2004).

Frequency modulations are more limited in calls of species living in a closed environment than in those of species ranging in a more open one between bushbabies species (Masters 1991) and between frog species of central Amazon (Zimmerman 1983). This is in line with general prediction (3). In contrast, among 95 Bolivian anuran species, the relationship between frequency modulations and vegetation density is inverted (Bosch \& de la Riva 2004), like in echolocation calls of 6 species of emballonurid bats (Kalko 1995). 
Table 2.3: Studies investigating environmental influences on acoustic variables of vocal signals in anurans and mammals. Vocalisation type: "C": call, "AdC": advertisement call, "AlC": alarm call, "LC": loud call, "EC": echolocation call. "yes": an influence of the environment in accordance with a general prediction (Table 2.1) was found, "no": no influence of the environment was found, "inv": an influence of the environment opposite to a general prediction (Table 2.1) was found.

\begin{tabular}{|c|c|c|c|c|c|c|c|c|c|c|c|c|c|c|}
\hline Study & $\begin{array}{l}\mathscr{0} \\
\frac{0}{0} \\
\stackrel{0}{0} \\
\text { की }\end{array}$ & 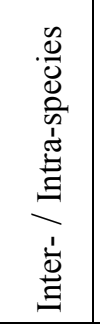 & 更 & 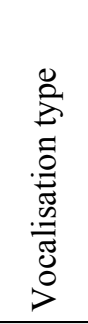 & 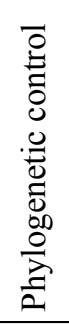 & 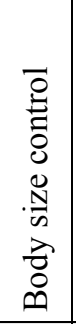 & 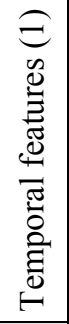 & 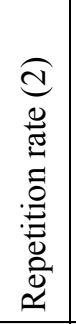 & 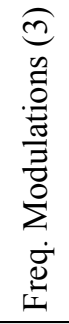 & 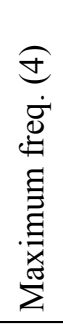 & 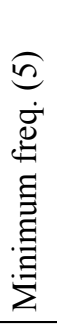 & 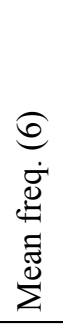 & 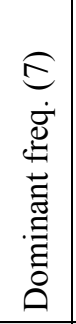 & 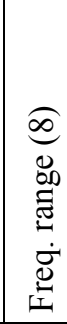 \\
\hline Bosch \& de la Riva 2004 & Anurans & Inter & 95 Bolivian anurans & $\mathrm{AdC}$ & no & no & no & no & inv & & & & no & no \\
\hline Kalko 1995 & Bats & Inter & 6 neotropical sheath-tailed bats (Emballonuridae) & $\mathrm{EC}$ & no & no & inv & & inv & & & inv & & \\
\hline Kalko \& Schnitzler 1993 & Bats & Intra & 4 Pipistrellus sp. & $\mathrm{EC}$ & yes & no & inv & & & & & & & inv \\
\hline Masters 1991 & Primates & Inter & Galago sp. & $\mathrm{LC}$ & no & no & inv & inv & yes & & & & & yes \\
\hline Mitani et al. 1999 & Primates & Intra & Pan troglodytes schweinfurthii & $\mathrm{LC}$ & no & yes & yes & yes & & yes & & yes & & \\
\hline Odendaal et al. 1986 & Anurans & Inter & Ranidella riparia vs. $R$. signifera & $\mathrm{C}$ & no & no & inv & yes & & & & no & & \\
\hline Perla \& Slobodchikoff 2002 & Rodents & Intra & Cynomys gunnisoni & $\mathrm{AlC}$ & no & no & no & & & inv & & yes & inv & \\
\hline Peters et al. 2008 & Felids & Inter & 6 Felis spp. & $\mathrm{C}$ & yes & yes & & & & & & no & inv & \\
\hline Siemers et al. 2001 & Bats & Intra & Myotis nigricans & $\mathrm{EC}$ & yes & no & inv & & & & & & & inv \\
\hline Sugiura et al. 2006 & Primates & Intra & Macaca fuscata yakui & $\mathrm{C}$ & yes & yes & & & & & & inv & & \\
\hline Wund 2005 & Bats & Intra & Myotis lucifugus & $\mathrm{EC}$ & yes & yes & inv & & & inv & & & & \\
\hline Zimmerman 1983 & Anurans & Inter & 56 central Amazon frog sp & $\mathrm{C}$ & yes & yes & & no & yes & & & & yes & \\
\hline
\end{tabular}


In accordance with general prediction (4), loud calls of chimpanzees tend to have a lower maximum frequency in primary rainforest than in more open habitat (woodland and secondary forest; Mitani et al. 1999). In contrast, in the pre-monsoon season, the maximum frequency of alarm calls of Gunnison's prairie dogs increases when vegetation cover increases (Perla \& Slobodchikoff 2002), and echolocation calls of little brown bats have a higher maximum frequency in a closed than in an open environment (Wund 2005).

No study examining the relationship between habitat features and minimum frequency has been found in anurans or mammals.

Mean frequency is lower in closed habitats than in more open ones in loud calls of chimpanzees (Mitani et al. 1999) and in alarm calls of Gunnison's prairie dogs in the postmonsoon season (Perla \& Slobodchikoff 2002). This is in line with general prediction (6). In contrast, coo calls of Japanese macaques have a lower mean frequency in open habitat than in primary or old secondary forest (Sugiura et al. 2006). Echolocation calls of 6 species of emballonurid bats also tend to have a lower frequency in species foraging in open habitats than in those foraging in cluttered habitats (Kalko 1995). No relationship between environmental characteristics and mean frequency has been found in calls of Ranidella riparia and $R$. signifera (Odendaal et al. 1986) and in mews of cat (sub)species (Peters et al. 2008).

Dominant frequency is lower in calls given by species living in closed habitats than in those of species living in more open habitats in central Amazon frogs (Zimmerman 1983). This is in accordance with general prediction (7). In contrast, alarm calls of Gunnison's prairie dogs present an increase in dominant frequency when vegetation cover increases in both pre- and post-monsoon seasons (Perla \& Slobodchikoff 2002). Similarly, intense mews of cat (sub)species living in densely vegetated habitats have a higher dominant frequency than those of (sub)species living in more open habitats (Peters et al. 2008). No relationship between environmental features and dominant frequency has been found in advertisement calls of Bolivian frog species (Bosch \& de la Riva 2004).

In accordance with general prediction (8), frequency range is narrower in calls of species living in closed habitats than in calls of species living in open habitats in bushbabies (Masters 1991) and in central Amazon frogs (Zimmerman 1983). In contrast, studies of echolocation calls in various bat species consistently highlight an opposite tendency (4 Pipistrellus species: Kalko \& Schnitzler 1993; black myotis: Siemers et al. 2001). No relationship between environmental features and frequency range has been found in advertisement calls of Bolivian frog species (Bosch \& de la Riva 2004). 


\subsection{CONCLUSIONS}

The present chapter evaluated the extent at which the vocal behaviour of birds as well as of anurans and mammals is adapted to the environment. Such adaptations appeared variable between species or environments and not as widespread as expected. From the limited number of studies collected, anurans and mammals were found to adjust call usage to the environment more often than birds. Conclusions from Boncoraglio and Saino (2007) concerning environmental influences on acoustic structure could not be extended to a set of additional studies. The only clear trends were a lower maximum frequency in closed than in open habitats, and no variation in repetition rate. Evidence for other acoustic variables remained unclear. Regarding environmental influences on acoustic structure in anuran and mammalian vocalisations, much fewer studies were found than in birds. Trends concerning acoustic variables preferentially adjusted to variations in environmental conditions remained therefore ambiguous.

\subsubsection{Usage}

Relatively few studies investigated environmental influences on usage of vocal signals, in birds as well as in anurans and mammals. Those conducted in birds highlighted less often environment-related variations in accordance with general predictions than those in anurans and mammals. However, this does not mean that flexibility in call usage in response to variations in environmental conditions is larger in anurans and mammals than in birds because the sample of available studies is small. Besides, independently of the taxa studied, isolating the effect of the environmental factor might be difficult. Other factors such as phylogenetic relationships, social system, context of calling, or internal state of the caller might interplay to regulate call usage (see Rendall et al. [2000] for an example of how travel rate might be related to bark rate more closely than habitat type in chacma baboons; Mays et al. 2006). For instance, other factors than favourable acoustic conditions (e.g., unfavourable foraging conditions, invasion of foreign intruders; Kacelnik \& Krebs 1983) might justify the dawn chorus in birds. Dabelsteen and Mathevon (2002), after failing to find favourable acoustic conditions at dawn, also suggested that social enhancement might trigger the dawn chorus in birds. Another example concerns call locations. Some primate species in Siberut primary forest (Kloss gibbons, Mentawai macaques, Mentawai leaf monkeys, pig-tailed 
langurs) give their loud calls from high posts, as predicted (Schneider et al. 2008). However, the authors suggested that food availability or some social factors might explain -as well as the acoustic properties of the environment- this spatial distribution for calling. All these considerations suggest that, despite the fact that it is seldom examined, adjustments in call usage to the gross habitat type is not as widespread as usually believed.

Birds, anurans and mammals might nevertheless adapt their calling times and places not to general features of the gross habitat type but to local conditions at the precise time and place (i.e., when and where) they vocalise. This suggests that local conditions should be evaluated each time when examining variations in call usage. This could also explain some contrasting results concerning the adjustment of calling times and places to the environment.

\subsubsection{Acoustic structure}

\subsubsection{Methodological points}

One should keep in mind (this concerns also call usage) that there might be a bias towards positive findings in published studies. Investigations highlighting variations in vocal behaviour in accordance with some general predictions might be more easily published and therefore more accessible than studies concluding on variations independent from the environment or contrary to general predictions. Thus, the present review might under-estimate the restriction of the "acoustic adaptation hypothesis", since publication of many studies revealing results not in line with general predictions might have failed.

An important methodological problem emerges while scanning the literature. Control of the recording distance lacks in many studies, especially in the oldest ones, although some acoustic variables are known to be modified during propagation, over about twelve meters (Fischer et al. 2002) or even less (Mathevon et al. 1996). Controlling recording distance would therefore lead to more reliable results, particularly when measuring acoustic variables related to energy distribution which are particularly sensitive to this factor.

Finally, another important methodological restriction is that only few studies were left in each category (birds vs. anurans and mammals, inter- and intra-species, levels of control for body size and phylogeny). This prevents drawing reliable conclusions for several acoustic variables, and might explain some of the weak tendencies found. 


\subsubsection{Comparison with the meta-analysis of Boncoraglio and Saino (2007)}

Overall, evidence from the sample of additional bird studies did not clearly corroborate results of the meta-analysis of Boncoraglio and Saino (2007). In accordance with general predictions, these authors found that birds tend to give vocalisations with lower minimum and dominant frequencies (a trend occurs in maximum frequency but disappears when phylogeny is controlled) and a narrower frequency range in closed than in open habitats, and that temporal features vary independently from the environment. In the additional bird studies, tendencies in accordance with general predictions were found only for maximum frequency, and there was just a slight trend for frequency range. Repetition rate was not varying in accordance with general prediction (2), but this was not tested by Boncoraglio and Saino (2007). For other acoustic variables, tendencies were ambiguous, probably because of the small number of studies which were considered in each category (see above). These results suggest that the pattern of variations related to environmental factors might be more complex than predicted. Acoustic variables adjusted to environmental conditions seem to depend upon the species and the environment considered.

All bird studies (those used in the meta-analysis and the additional ones, from all categories) can be pooled (Table 2.2). There appears to be a trend for lower minimum frequency, less frequency modulations, and narrower frequency range in closed than in open habitats, as predicted. No influence of the habitat occurs on temporal features, repetition rate and maximum frequency. Results are ambiguous for mean frequency (small sample of studies) and dominant frequency. Confirmation of general prediction (8) for frequency range corroborates the assumption that this acoustic variable is more dependent on ecological factors than on phylogeny (McCracken and Sheldon 1997). Reserved results for temporal features, repetition rate and maximum frequency suggest that only some acoustic variables (e.g., frequency range) are often adjusted to the environment. The other ones (e.g., duration) might be more dependent on other factors, such as phylogeny, call context, caller arousal or short-term variations in social surrounding or ambient noise level. For instance, short-term increases in ambient noise level lead to a lengthening of calls as a by-product of the Lombard effect (Lombard 1911; e.g., tree swallows [Tachycineta bicolor]: Leonard \& Horn 2005). These short-term variations can be tested when the same individuals or groups are studied under various environmental or social conditions. Besides, optimising sound propagation does not automatically imply increasing propagation distance. Some signals might be intended to degrade in a determined way or to carry only up to a limited distance to favour distance 
estimation or reduce eavesdropping from predators (see General introduction). This might also explain some reserved results.

Tendencies emerging in the extended sample of bird studies (all bird studies pooled) did not seem to be affected by the fact that calls (3 studies) or songs (39 studies) were examined, or by the level of control (phylogeny, body size). Results in accordance with or against general predictions were found for calls and songs and at all levels of control. The fact that studies were conducted in passerine species (35 studies), non-passerine species ( 3 studies) or a mix of both (4 studies) also did not seem to affect the outcome of the survey, even if Morton (1986) suggested that adaptation to the local habitat should be stronger in songs of passerine birds. Indeed, passerine birds have little energy storage and cannot afford to react to another bird which is not a threat. They might therefore try to repel neighbours by uttering songs which propagate far without degradation (Morton 1986). In fact, results in line with general predictions were not more often found in passerine species than in other bird species. For instance, in New World doves (Tubaro \& Mahler 1998), almost all acoustic variables were varying with the environment contrary to general predictions. However, this might not be linked to the fact that these were non-passerine bird species, since no relationship between habitat features and acoustic structure (e.g., 40 South African passerine species: Saunders \& Slotow 2004; 121 Australian songbird species: Blumstein \& Turner 2005; dark-eyed juncos [Junco hyemalis]: Slabbekoorn et al. 2007) or results contrary to general predictions (e.g., rufous-collared sparrows [Zonotrichia capensis]: Handford \& Lougheed 1991; indigo buntings [Passerina cyanea]: Hylton \& Godard 2001) were also found in passerine species. Hansen (1979) assumed that vocal learning might have favoured environmental adaptation since young birds would preferentially learn songs which propagate well. However, the occurrence of vocal learning did not seem to play a determinant role in adjustment of call structure to the environment. For instance, predictions were verified in a group of tinamous species which lack vocal learning (Bertelli \& Tubaro 2002), while they were not verified in indigo buntings (Hylton \& Godard 2001) and a group of 121 Australian songbird species (Blumstein \& Turner 2005) which are song learners. These different factors (songs, passerines, vocal learning) which do not seem to influence environment-related variations suggest that environmental adaptations might have occurred independently over evolution in bird taxa. 


\subsubsection{Studies in anurans and mammals}

Much fewer studies examining the relationship between acoustic structure and environmental features were found in anurans and mammals than in birds. This could explain the unclearness of trends found in the present review. Only the study of Mitani and colleagues (1999) showed results consistently in line with general predictions (1), (2), (4) and (6). Other studies always presented at least one acoustic feature in which variations were not in accordance with general predictions. Conclusions about acoustic variables which are preferentially adapted to environmental conditions cannot then be drawn for anurans and mammals.

However, in most studies, results not in line with general predictions clearly suggest the importance of other potentially influential factors. Concerning the study of Masters (1991) in two Galago species, reversed trends in temporal features and repetition rate might reflect adjustments to turbulence level in each habitat type and not to vegetation density. In addition, body size might blur the effect of the environment on temporal parameters (Masters 1991). Results opposite to general predictions in Felis (sub)species might also be explained by an interplay with body size (whose effect on vocal signals [Peters et al. 2008] is already opposite to what theory predicts [Fitch \& Hauser 1995]). Further investigations on precise acoustic features (e.g., propagation conditions, ambient noise) of the various habitat types are also needed to test for local adaptation (Peters et al. 2008). Such particular habitat-specific acoustic properties explain the trend opposite to general prediction (6) in mean frequency of coo calls in two populations of Japanese macaques (Sugiura et al. 2006). The lower mean frequency of coo calls uttered in the open habitat type is consistent with a better transmission of low frequencies in this open habitat than in forest at this particular study site (Sugiura et al. 2006). Acoustic features of anuran calls varied with the environment in line with predictions, contrary to predictions or not at all. This diversity in the direction of variations might be explained by different factors. In the study of Zimmerman (1983), variations could be explained by body size. Taxonomy might also be a major explicative factor and microhabitat features might explain accurately some variations in frog calls (Bosch \& de la Riva 2004). Studies in bat species consistently highlighted habitat-related variations in duration, pulse interval and bandwidth contrary to general predictions (1) and (8) (Kalko \& Schnitzler 1993; Kalko 1995; Siemers et al. 2001; Wund 2005). However, these variations are linked to the function of echolocation calls used during foraging, in comparison to communicative signals in other taxa (Kalko 1995). For instance, calls with a large bandwidth in cluttered habitats are adequate to distinguish prey echoes from background targets (i.e., vegetation) which should 
be precisely located to avoid collision during hunting. A narrow bandwidth in open habitat is well-adapted to detect prey and locate them in an environment free of vegetation (Kalko \& Schnitzler 1993; Siemers et al. 2001). Finally, variations with vegetation cover contrary to general predictions (4) and (7) in alarm calls of Gunnison's prairie dogs can be explained by the season considered. Calls are not intended to travel far in the environment when young animals stay near the burrows in the pre-monsoon season (Perla \& Slobodchikoff 2002). When variations in fundamental frequency correspond to general prediction (6) in the postmonsoon season, it might be because calls are intended to travel further when young animals begin to go away of the burrows. These seasonal changes should limit transfer of information about the group's location to potential predators (Perla \& Slobodchikoff 2002). These last two examples in bats and in prairie dogs highlight that the signal function should be taken into account while elaborating predictions on environment-related variations in acoustic structure of calls.

\subsection{PERSPECTIVES}

To sum up, isolating the effect of the environment on usage of vocal signals might sometimes be difficult and local variations might play an important role. Variations in acoustic structure of vocal signals seem to be dependent on the species, the environment and the function of the calls considered. Predictions for environmental influences on vocal behaviour should therefore be adapted to the peculiarities of the study species and its environment. From these results, two perspectives for future studies can be drawn.

Environmental factors vary within similar gross habitat types. Animals might therefore adjust their vocal behaviour to local conditions and not to general environmental features, as suggested by the amount of ambiguous evidence for usage and acoustic structure. For instance, studies in rufous-collared sparrows highlighted different tendencies in environmental-related variations in songs of different populations (Nottebohm 1975; Handford 1981, 1988; Handford \& Lougheed 1991; Tubaro et al. 1993; Tubaro \& Segura 1994; Kopuchian et al. 2004; Lijtmaer \& Tubaro 2007; see Appendix 2). This suggests that local conditions might differ between populations. A precise characterisation of each habitat should then precede any study on the influence of the habitat on vocal behaviour (Eyring 1946; Bosch \& de la Riva 2004; Saunders \& Slotow 2004). More particularly, the exact calling locations should be described (McCracken \& Sheldon 1997; Saunders \& Slotow 2004). This includes for instance altitude, humidity, temperature, local avifauna, vegetation 
structure, and even historical evolution of the habitat (Nottebohm 1975; Handford 1981, 1988; Sorjonen 1986b; Handford \& Lougheed 1991; Doutrelant \& Lambrechts 2001; Sugiura et al. 2006). Such precise information might also help to understand whether and how some particular factors enhance adaptations in some acoustic variables in some species but in other variables in other species.

Future investigations should improve the level of control for other factors and concentrate on other vertebrate taxa than birds. An ideal way of further investigating the "acoustic adaptation hypothesis" would be to record the same individuals in different environmental conditions, like in studies of the Lombard effect in birds (e.g., Japanese quails [Coturnix coturnix japonica]: Potash 1972) and in primates (e.g., common marmosets: Brumm et al. 2004; cotton-top tamarins: Egnor \& Hauser 2006). Rendall and colleagues (2000) used this method to test the influence of habitat openness on bark rate of chacma baboons. However, only one study (captive little brown bats: Wund 2005) used these testconditions to investigate the effect of a more or less obstructed environment on the acoustic structure of vocal signals. Such test-conditions should document the intra-individual degree of plasticity in vocal behaviour in response to environmental conditions (see Chapter 4). 


\title{
Chapter 3
}

\section{Habitat characterisation - A suggestion for standardised methods and an example of application}

\begin{abstract}
Each environment has its own acoustic properties, leading to different rates of attenuation and distortion of acoustic signals. Knowing these properties is necessary to examine whether animals adjust usage and structure of their acoustic signals to optimise propagation (Chapter 2). However, the diversity of methods used up to now to describe habitat types might be confusing. Therefore, the present chapter suggests some standardised methods to characterise both structural (tree density, visual obstruction) and acoustic (ambient noise, attenuation rate) properties of the environment. These methods aim to be mainly used in comparative studies. As an example of application, two types of habitats, namely forest and woodland savannah, were characterised. Tree density and visual obstruction by vegetation were measured and compared between habitat types. The sources of ambient noise, its frequency ranges, its amplitude level and its energy distribution were examined in both habitat types. Finally, sine waves were broadcasted to quantify frequency-dependent attenuation in forest and woodland savannah. Methods described in the present chapter are easy to put into practice and confirm results of previous studies, for instance the more important attenuation rate of high frequencies in closed than in open habitats. Combining these different methods gives an overview of the main features of each habitat type to which usage and structure of acoustic signals used in animal communication might be adapted.
\end{abstract}

Kurt Hammerschmidt commented earlier drafts of this chapter. 


\subsection{INTRODUCTION}

Each environment has its own characteristics: substances forming the medium (e.g., atmosphere, water), substances constituting the boundaries (e.g., soil) and objects of different impedance within the medium (e.g., drops of humidity, leaves, stems, moving column of air). These features affect sound propagation. They determine the rate of absorption (e.g., Piercy et al. 1977; Wiley \& Richards 1978; Fricke 1984) and reverberation (due to scattering; e.g., Piercy et al. 1977; Richards \& Wiley 1980; Waser \& Brown 1986; Naguib \& Wiley 2001) of sounds travelling through this environment. These processes lead to sound attenuation and distortion (Chapuis 1971; Aylor 1972; Marten \& Marler 1977; Marten et al. 1977; Piercy et al. 1977; Richards \& Wiley 1980; Waser \& Brown 1986; Date \& Lemon 1993; Forrest 1994; Naguib \& Wiley 2001; Naguib 2003; Nelson 2003; Padgham 2004). Attenuation stems from two sources. One is global attenuation, which is due to frequency-independent spherical spreading (Wiley \& Richards 1978; Forrest 1994; Bradbury \& Vehrencamp 1998). The other one is excess attenuation, which is the frequency-dependent difference between the amplitude expected after spherical spreading at a distance from a source and the measured amplitude at that distance (Price et al. 1988; Forrest 1994). Distortion results from structural modifications in the time domain (i.e., in temporal parameters of a signal), because of reverberation.

In addition to absorption and reverberation, ambient noise also affects acoustic communication by restricting signal detection. It is due to biotic sources (i.e., acoustic signals of animals; de la Torre \& Snowdon 2002; Slabbekoorn 2004), and / or to non-biotic sources, such as the interaction between turbulent air and vegetation (Waser \& Brown 1986; Forrest 1994; Blumenrath \& Dabelsteen 2004) or noise related to human presence (Snowdon \& de la Torre 2002). Non-biotic sources (e.g., wind) create mainly ambient noise with low frequencies (between 100 and $200 \mathrm{~Hz}$ ), much lower than those from bird and insect sounds (between $1000 \mathrm{~Hz}$ and $7000 \mathrm{~Hz}$, but mostly between $4000 \mathrm{~Hz}$ and $7000 \mathrm{~Hz}$; Ryan \& Brenovitz 1985; Waser \& Brown 1986). Ambient noise varies with habitat type, season, time of day, distance and height (moist evergreen forest: Waser \& Waser 1977; riverine forest, rainforest and savannah: Waser \& Brown 1986; deciduous temperate forest: Dabelsteen \& Mathevon 2002; deciduous forest: Blumenrath \& Dabelsteen 2004; ecotone forest and rainforest: Slabbekoorn 2004; primary rainforest: Schneider et al. 2008).

Absorption, reverberation and ambient noise impose therefore constraints specific to each environment on propagation and detection of acoustic signals (e.g., Ingard 1953; Embleton 1963; Linskens et al. 1976; Forrest 1994). According to the "acoustic adaptation 
hypothesis", animals might adjust both usage (time, height of signalling post, call rate; e.g., Waser \& Waser 1977), and structure (e.g., Chapuis 1971; Morton 1975) of their acoustic signals to optimise transmission efficiency (see Chapter 2). Knowing environmental characteristics would then help to formulate hypotheses on potential adaptations of acoustic signals and to interpret variations in the vocal behaviour of animals ranging in different habitat types (Chapter 2). A gross description of habitat types -such as "open / closed"- is insufficient, since absorption, reverberation and ambient noise are tightly linked to local environmental characteristics (e.g., atmospheric conditions, vegetation structure, bird and insect communities). A finer characterisation is therefore needed to understand peculiarities of the habitat types under study. However, studies examining the adjustment of vocal signals to the habitat in for instance anurans, birds and mammals characterised either different environmental aspects (e.g., presence vs. absence of measures of sound attenuation: Waser \& Brown 1986 vs. Schneider et al. 2008) or the same environmental aspects but with slightly different methods (e.g., measures of sound attenuation with different frequencies and distances: Richards \& Wiley 1980 vs. Waser \& Brown 1986; measures of ambient noise level with different calibrations and values [mean vs. maximum / minimum]: Waser \& Brown 1986 vs. Schneider et al. 2008).

The present chapter suggests therefore standardised methods to characterise main environmental aspects, with the long-term aim of enhancing a certain degree of uniformity between studies. First, a way of characterising some structural properties of habitat types is presented. Tree density, which is globally constant across seasons, is used to define gross habitat categories. As this measure does not take into account seasonal structural variations, visual vegetation density (i.e., visual obstruction because of vegetation) which is likely to vary with seasons is evaluated. Second, methods to characterise the acoustic environment are described. The frequency range of ambient noise, its sound pressure level and its energy distribution are evaluated to find out which habitats are most favourable to acoustic communication, that is, with low ambient noise level and some frequency ranges relatively free of ambient noise. (Ambient noise does not include primate vocalisations since the present chapter aims to characterise the acoustic environment for vocal communication in baboons [see conclusions].) In addition, broadcasting pure tones (sine waves) allows estimation of frequency-dependent attenuation in the different habitat types. The present methods quantify sound modifications due to propagation in the amplitude and frequency domains, but not any variations in their temporal features. They are used in forest and woodland savannah as an example of application to provide an overview of what kind of information can be extracted. 
Finally, they are commented and discussed in the light of previous studies characterising habitats.

\subsection{MATERIAL AND METHODS}

\subsubsection{Study sites}

Methods described hereafter were applied in Gashaka-Gumti National Park, eastern Nigeria $\left(6^{\circ} 58^{\prime}-8^{\circ} 13^{\prime} \mathrm{N} ; 11^{\circ} 13^{\prime}-12^{\circ} 11^{\prime} \mathrm{E}\right)$. This park of about $6400 \mathrm{~km}^{2}$ near the border with Cameroon is divided into two sectors: the Gumti sector in the north (Adamawa state) and the Gashaka sector in the south (Taraba state; Harcourt \& Ellerton 1995; Akinsoji 1996). Two study sites were characterised: one near the village of Gashaka along the river Gamgam (Gamgam, $1.48 \mathrm{~km}^{2}$; Warren 2003) and one around Kwano field station (Kwano, $1.49 \mathrm{~km}^{2}$; Warren 2003). Both are located in the Gashaka sector, which is mostly hilly and drained by many rivers (Chapman \& Chapman 2001). Altitudes range between $300 \mathrm{~m}$ and $2400 \mathrm{~m}$ (Dunn 1993; Harcourt \& Ellerton 1995). Two main seasons are clearly distinguishable: a rainy season from April to mid-November and a dry season from mid-November to March (Harcourt \& Ellerton 1995; Akinsoji 1996). Data were collected in the dry season (Nov 06 Mar 07). The annual rainfalls were of $2079 \mathrm{~mm}$ in Gamgam and $2033 \mathrm{~mm}$ in Kwano in 2006. In the same year, the minimum temperatures were $22^{\circ} \mathrm{C}$ and $20^{\circ} \mathrm{C}$ and the maximum temperatures were $31^{\circ} \mathrm{C}$ and $32^{\circ} \mathrm{C}$ in Gamgam and Kwano, respectively (GPP weather station).

The study area is located in the Guinea savannah zone, but it nevertheless includes a mosaic of vegetation types (Harcourt \& Ellerton 1995; Akinsoji 1996). Forest and southern Guinea savannah (hereafter woodland savannah) are the dominant ones in the two study sites. Forest is located mainly on foothills, where rivers and streams run over the terrain. It includes emergent trees ( $32 \mathrm{~m}$ high and sometimes even higher), trees from the canopy stratum, understorey trees, shrubs, ferns, lianas, old farm species, and herbs (Akinsoji 1996). Forest patches in Gamgam were mostly represented by riverine forest along seasonal streams, whereas in Kwano lowland rainforest constituted most forested areas, which nevertheless also included a few parts of riverine forest. Woodland savannah includes woody species not as tall as in forest because of burning, woody climbers, grass up to $4 \mathrm{~m}$ high, and species specific to stream sides. Its composition differs according to soil characteristics, temperature, intensity of fires and biotic influences (Akinsoji 1996). 


\subsubsection{Sampling points}

To characterise the two main habitat types (forest and woodland savannah), 15 points per habitat type (i.e., 30 points per study site) were determined on a map of each site (Warren 2003). The two following methods were used to position the sampling points, since woodland savannah was predominant in Gamgam and forest in Kwano (patches of the other habitat type were interspersed in these dominant habitat types).

Three longitudinal lines (NW - SE) were drawn on a map of Gamgam site. On each of them, five points were distributed as equidistantly as possible in woodland savannah. Eight transversal lines (SW - NE) were then drawn and 15 points were positioned in forest, as close as possible to an intersection with a longitudinal line.

Five longitudinal lines (SW - NE) were drawn on a map of Kwano site. On each of them, three points were distributed in forest. More than three points in forest were placed on some lines, depending on habitat patchiness. Then, four transversal lines (NW - SE) were drawn and 15 points were positioned in woodland savannah as close as possible to an intersection with a transversal line.

The GPS positions of these points in the field were saved (Garmin GPSMAP 60CSX; Garmin, United States of America). These points were used to evaluate tree density and visual vegetation density. Recordings for acoustic characterisation were done only at one of these points per habitat type. Measurements were repeated 3 times (i.e., 3 sessions were conducted) at the same points, as grass height varied throughout the dry season in woodland savannah: when the grass was high ("High grass": mid-November, beginning of dry season), when the grass was low because of recent burning ("Low grass": mid-January, middle of dry season) and when the grass began to re-grow ("Re-growing grass": mid-March, end of dry season). Tree density estimations were not repeated, because the trees considered were too large to be affected by burning.

\subsubsection{Vegetation density}

\subsubsection{Tree density}

Vegetation with a woody stem was considered as a tree when diameter at breast height $(\mathrm{DBH})$ was larger than $5 \mathrm{~cm}$. Four tree categories were considered, according to diameter at breast height: $5 \leq \mathrm{DBH}<10 \mathrm{~cm}, 10 \leq \mathrm{DBH}<20 \mathrm{~cm}, 20 \leq \mathrm{DBH}<30 \mathrm{~cm}$, and $\mathrm{DBH} \geq 30 \mathrm{~cm}$. The point-quarter sampling method (James \& Shugart 1970; Ganzhorn 2003) used here can be summarised as follows. At each point, the four compass directions divided the sampling area 
into four quarters. In each quarter and for each tree category, the distance from the centre of the nearest tree to the sampling point was measured (the trunk radius was added to the distance measured in the field). Densities for each tree category at each point were then calculated by dividing a unit area $\left(10000 \mathrm{~m}^{2}\right)$ by the square of the mean distance between a tree and the sampling point.

At several points, impenetrable forest undergrowth (especially in Gamgam), elephant grass (Panicum maximum, only in Gamgam), or a too steep slope (especially in Kwano) prevented any measurements. Tree density could then be measured in 11 points in forest and 14 points in woodland savannah in Gamgam, and in 14 points in forest and 15 points in woodland savannah in Kwano. In a few quarters, some trees were not accessible (e.g., gap) or not visible, impeding measurements of the distance between the tree and the sampling point. Tree density for these points was therefore calculated from the mean distance of a tree to the sampling point over less than 4 measures.

This method has been shown to reflect reliably relative structural differences between habitat types (Ganzhorn 2003). Only data about total tree density, without taking into account size category, are presented here. Such data are sufficient in the present example of application to build gross habitat categories. A finer determination of tree density for each size class might be more useful when comparing variants of the same gross habitat types (see discussion). Tree densities were compared between forest and woodland savannah using exact 2-tailed Mann-Whitney U-tests. This statistical analysis and all the following ones were conducted with the software SPSS 15.0 for Windows. The significance level was set at 0.05 .

\subsubsection{Visual vegetation density}

At least ten points per habitat type were visited in each site in each session (points in forest were visited only once, since forest was not noticeably changing over the dry season). At each point, a piece of white tissue (approximately $1.5 \times 2.0 \mathrm{~m}$ ) was held above ground and a photograph of it was taken with a digital camera Canon Powershot A520 (Canon, Japan; focal distance: $5.8 \mathrm{~mm}$ ) from $5 \mathrm{~m}$ and $12.5 \mathrm{~m}$ from north and south at $1.5 \mathrm{~m}$ above ground (i.e., eye level and within high grass). Two pictures per point per session and per distance were then obtained.

Visual obstruction was estimated by quantifying the remaining visible area of the piece of tissue through vegetation (de la Torre \& Snowdon 2002). For this, each digital photograph was modified with the software Photoshop CS 8.0.1 (Adobe, United States of America). The "view" image represented the remaining visible area of the piece of tissue 
coloured in black, and the surrounding, including vegetation obstructing the piece of tissue, coloured in white. From the "view" image, a "reference" image was built by colouring in black the estimated whole area of the piece of tissue, including parts previously obstructed by vegetation; background remained white. The amount of black pixels was measured on both the "view" and "reference" images with the software Photoshop CS 8.0.1 (Histogram: Luminanz). The ratio (in percentages) of these quantities was then calculated between the "view" and "reference" images for each original picture. These ratios were then averaged for each distance at each point. These averaged ratios were compared between forest and woodland savannah in the three sessions (high, low and re-growing grass) by using exact 2tailed Wilcoxon T-tests. Data were not independent since measures were repeated in three sessions in woodland savannah.

\subsubsection{Acoustic environment}

\subsubsection{Ambient noise}

Methods were inspired from those used by Schneider and colleagues (2008), but recording times were distributed differently over the day. In each study site, one point representative of each habitat type was chosen among the ones used to characterise vegetation density. These points were not too far from one another, so that they could be reached within one hour. In each habitat type and for each hour of the morning (between 6:00 and 12:00: hour 6 , hour 7 , hour 8 , hour 9, hour 10, hour 11 [hour $6=$ from 6:00 to 6:59]), ambient noise was recorded with a digital solid-state recorder (Marantz PMD660; Marantz, Japan; sampling frequency: $44.1 \mathrm{kHz}$; 16-bit resolution; mono) and a Sennheiser directional microphone (K6 power module and ME66 recording head with a Rycote softie windscreen; Sennheiser Electronic KG, Germany) held $1 \mathrm{~m}$ above ground. Recordings lasted $90 \mathrm{~s}$ in each of the four cardinal directions to take into account variations due to microhabitat fluctuations (de la Torre \& Snowdon 2002). At the same time, ambient noise level was measured with a Voltcraft sound pressure level meter 322 Datalogger (Voltcraft, Germany; linear weighting ' $\mathrm{C}$ ', format: auto). Maximum and minimum sound pressure levels (SPL) in each period of $90 \mathrm{~s}$ were measured.

Quantitative variations in ambient noise level throughout the morning were described in both habitat types. The mean between maximum and minimum SPL was calculated for each hour of the morning and for each habitat type. Ambient noise levels were compared between habitat types across sessions and within each sessions using exact 2-tailed Wilcoxon 
T-tests, since data were not independent (repetitions over sessions, hours and in the four cardinal directions).

Spectrograms (sampling frequency: $44.1 \mathrm{kHz}$; FFT-length: 1024 points; frequency resolution: $86.3 \mathrm{~Hz}$; Hamming window; overlap: $50 \%$; time resolution: $11.6 \mathrm{~ms}$ ) of audiorecordings were calculated with the software Avisoft SASLab Pro recorder 4.3 (R. Specht, Berlin, Germany). They were used to describe sources (insects, birds, frogs, wind) and frequency ranges of ambient noise. Four periods of $1 \mathrm{~s}$ were then selected in each audiorecording of $90 \mathrm{~s}$ (one period of $1 \mathrm{~s}$ in each quarter of the audio-recording of $90 \mathrm{~s}$ ) on the main window. Binary spectrograms were then exported to the software LMA 2007 developed by K. Hammerschmidt (Schrader \& Hammerschmidt 1997). The energy distribution was calculated from the spectrograms of each period of $1 \mathrm{~s}$ (sampling frequency: $44.1 \mathrm{kHz}$; FFT-length: 1024 points; frequency resolution: $86.3 \mathrm{~Hz}$; Hamming window; overlap: $50 \%$; time resolution: $11.6 \mathrm{~ms}$ ) using the general macro of LMA 2007 (cut-off frequency: $0 \mathrm{~Hz}$; start and end amplitude thresholds: $1 \%$; noise factor: 1.5 ; repetition factor: 1 ; amplitude calculation mode: general; no filter; contour threshold: 0.2; F0 modulation limit: $30 \%$; F0 range for IM: 0.5). Mean peak frequency (i.e., frequency with the highest amplitude) and frequencies at which $25 \%$ (dfa1), $50 \%$ (dfa2) and $75 \%$ (dfa3) of the global energy were reached were calculated. These data were used to examine which frequency ranges concentrated most energy and how frequency distribution of ambient noise varied in the morning course.

\subsubsection{Test of attenuation}

The same points as those visited for recording ambient noise were used. However, in Gamgam, one point was added in woodland savannah, where grass was first very high and then completely burnt. In Kwano, the point in forest was replaced by a completely new one also in forest but along the footpath, where distance could be measured more easily. One point was added in woodland savannah, where grass evolved naturally without burning this year (grass was dry and down in January). These new points were also representative of the main habitat types. Experiments were conducted between 8:00 and 9:00 in the morning (average ambient noise) on a day with no or very little wind.

A synthetic signal, generated with the software Cool Edit 2000 (now Adobe Audition; Syntrillium Software Corporation, United States of America), was composed of 14 pure tones, each lasting $3 \mathrm{~s}$, separated by periods of silence of $2 \mathrm{~s}$, and of decreasing frequency: 16000, 14000, 10000, 8000, 6000, 4000, 2000, 1000, 800, 600, 400, 200, 100 and $50 \mathrm{~Hz}$. The loudspeaker (Visonik Davidactive; Visonik HiFi, Germany) was positioned $1 \mathrm{~m}$ above 
ground, facing south-west in Gamgam, and east in woodland savannah and west in forest in Kwano. Synthetic signals were re-recorded from $1 \mathrm{~m}$ (reference without sound distortion), $5 \mathrm{~m}, 12.5 \mathrm{~m}, 25 \mathrm{~m}$, and $50 \mathrm{~m}$ at each point with a digital solid-state recorder (Marantz PMD660; Marantz, Japan; sampling frequency: $44.1 \mathrm{kHz}$; 16-bit resolution; mono) and a Sennheiser directional microphone (K6 power module and ME66 recording head with a Rycote softie windscreen; Sennheiser Electronic KG, Germany) held $1 \mathrm{~m}$ above ground.

Three periods of $50 \mathrm{~ms}$ were chosen for each pure tone (i.e., each frequency), with one 50-ms-period in each third of each pure tone. Sections with background noise were avoided. For each 50-ms-period, a power spectrum (logarithmic scale) was calculated with the software Avisoft (sampling frequency: $44.1 \mathrm{kHz}$; FFT-length: 1024 points; Hamming window; bandwidth: $25.5 \mathrm{~Hz}$; resolution: $10.8 \mathrm{~Hz}$ ). Amplitude of the frequency corresponding to the pure tone was measured with the free horizontal cursor function. The difference between the mean amplitude of a frequency recorded at $1 \mathrm{~m}$ from the loudspeaker and the amplitude of the same frequency recorded at $5 \mathrm{~m}, 12.5 \mathrm{~m}, 25 \mathrm{~m}$ or $50 \mathrm{~m}$ from the loudspeaker was then plotted as a function of frequency. These attenuation profiles were compared between habitat types.

\subsection{RESULTS}

\subsection{1 $\quad$ Tree density}

In both study sites, mean $( \pm \mathrm{SE})$ tree density was significantly higher in forest (Gamgam: $983 \pm 202$ trees / ha; Kwano: $1225 \pm 143$ trees / ha) than in woodland savannah (Gamgam: $517 \pm 84$ trees / ha; Kwano: $887 \pm 230$ trees / ha; exact Mann-Whitney U-tests: Gamgam: $\mathrm{N}_{1}=11, \mathrm{~N}_{2}=14, \mathrm{U}=39, \mathrm{p}=0.038$; Kwano: $\mathrm{N}_{1}=14, \mathrm{~N}_{2}=15, \mathrm{U}=58, \mathrm{p}=0.041$; Figure 3.1). 


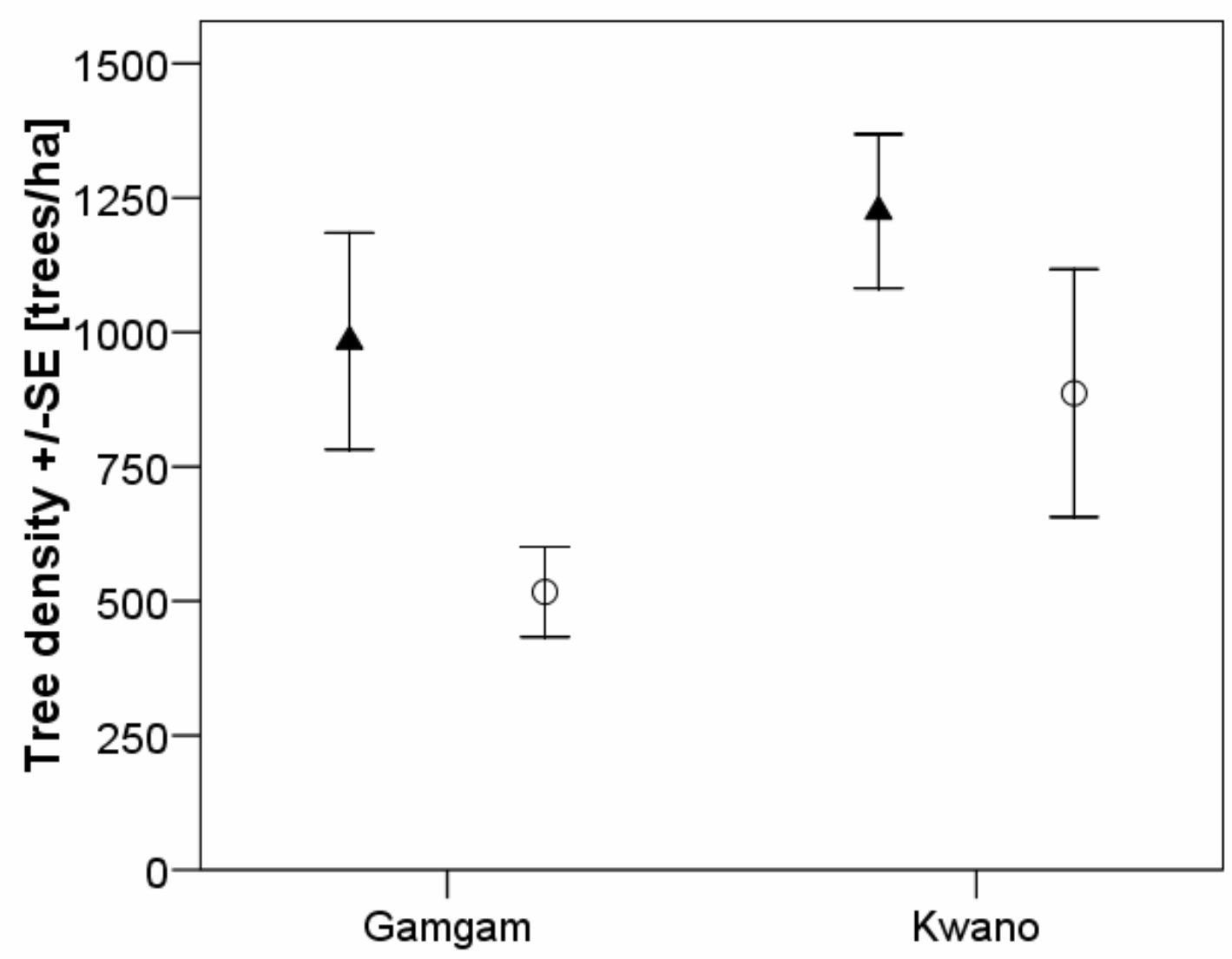

Figure 3.1: Mean tree density and its standard error in forest (triangles) and in woodland savannah (circles) in Gamgam and Kwano.

\subsubsection{Visual vegetation density}

Only analyses of pictures taken from $5 \mathrm{~m}$ are presented. Analyses of pictures taken from $12.5 \mathrm{~m}$ led to similar differences between habitat types. In Gamgam (Figure 3.2a) as in Kwano (Figure 3.2b), two distinct categories could be built according to visibility. Forest and woodland savannah with high grass presented a small visible proportion of the piece of tissue (i.e., a low visibility) which was not significantly different between one another; these habitat types constituted the first category (closed habitats). Significant differences occurred with the second category (open habitats), including woodland savannah with low and re-growing grass. These habitats were less visually obstructed than the previous ones. 
a.

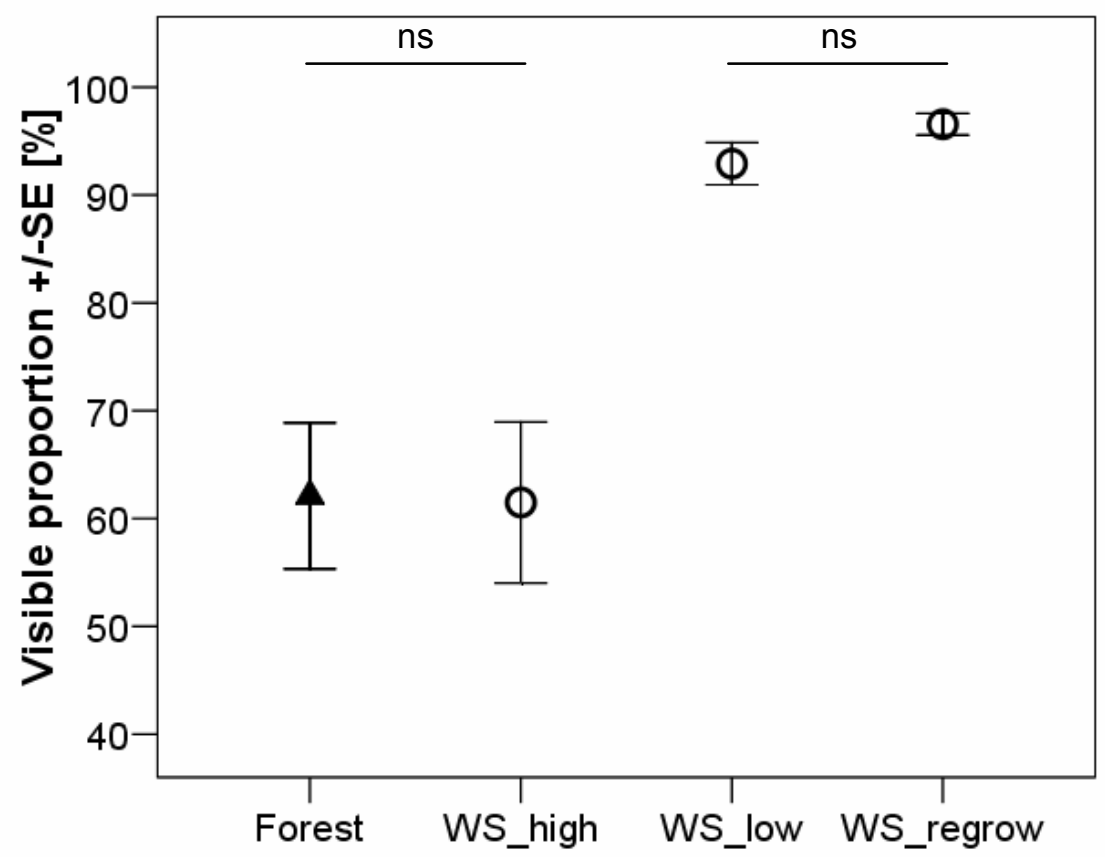

b.

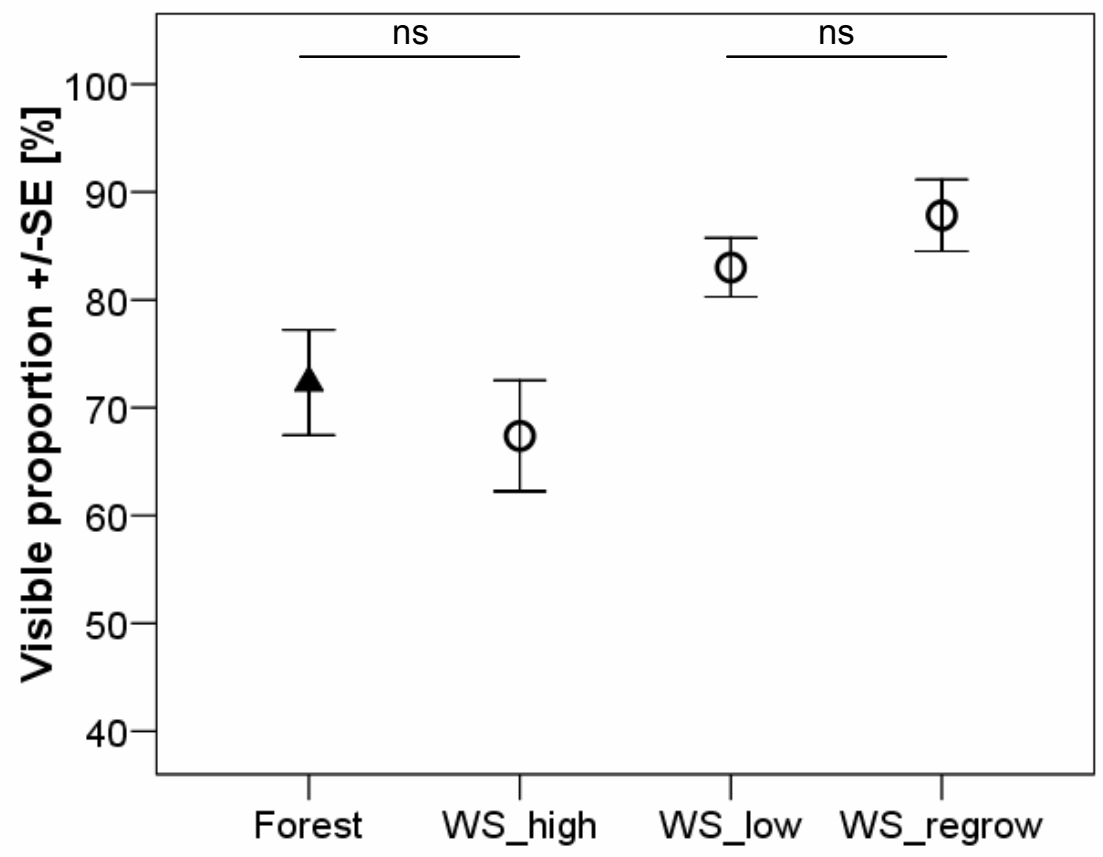

Figure 3.2: Mean visible proportion (\%) and its standard error of a piece of tissue through vegetation from $5 \mathrm{~m}$ in forest, woodland savannah with high grass (WS_high), woodland savannah with low grass (WS_low) and woodland savannah with re-growing grass (WS regrow) in (a) Gamgam and (b) Kwano. Comparisons were done with exact 2-tailed Wilcoxon T-tests. Differences between habitat types obtained from analyses of pictures taken from $12.5 \mathrm{~m}$ were similar. 


\subsection{3 $\underline{\text { Ambient noise }}$}

\subsubsection{Sources of ambient noise}

Visual inspection of spectrograms revealed that ambient noise was mostly due to insects, birds and non-biotic sources such as wind in both habitat types. Several insect species gave acoustic signals in very narrow frequency ranges, for instance around $1100 \mathrm{~Hz}, 2800 \mathrm{~Hz}$ and $7300 \mathrm{~Hz}$ (Figure 3.3a). Acoustic signals of other insects concentrated their energy between $4000 \mathrm{~Hz}$ and $8000 \mathrm{~Hz}$, with some peaks up to $16000 \mathrm{~Hz}$ (Figure 3.3b). Most bird species gave calls between $250 \mathrm{~Hz}$ and $4000 \mathrm{~Hz}$ (sometimes up to $6000 \mathrm{~Hz}$ and even $11000 \mathrm{~Hz}$ in a few cases; Figure 3.3c and d). Non-biotic noise (e.g., wind) was predominant in low frequencies, under $1000 \mathrm{~Hz}$, and very rarely up to $3200 \mathrm{~Hz}$ (Figure 3.3d).
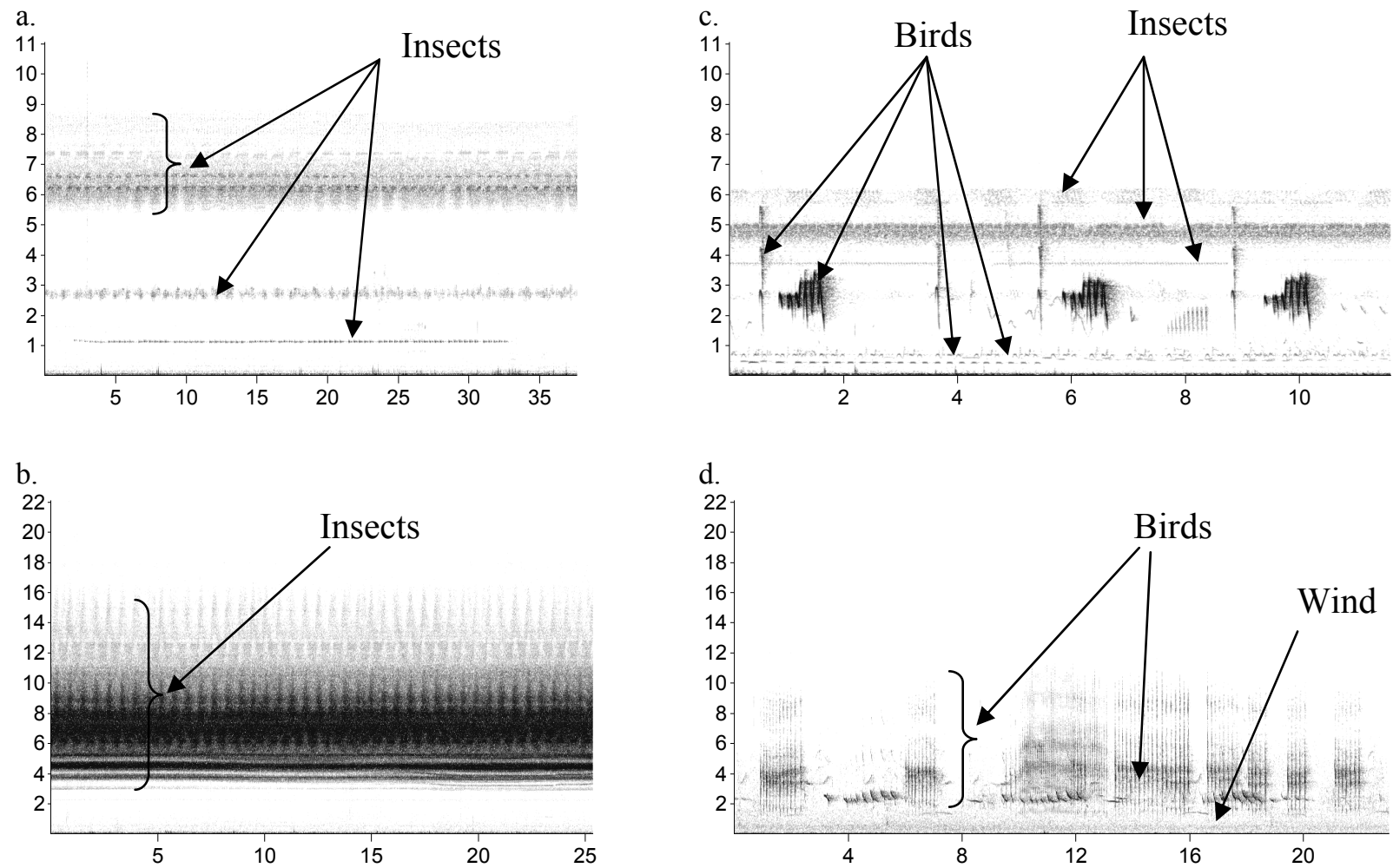

Figure 3.3: Spectrograms of recordings of ambient noise in Kwano and Gamgam at different times of the morning and of the dry season (X-axis: Time [s]; Y-axis: Frequency [kHz]). (a) Hour 9 in woodland savannah with high grass in Kwano (sampling frequency: $22.05 \mathrm{kHz}$; 16-bit resolution). (b) Hour 9 in woodland savannah with re-growing grass in Kwano (sampling frequency: $44.1 \mathrm{kHz}$; 16-bit resolution). (c) Hour 7 in woodland savannah with high grass in Gamgam (sampling frequency: $22.05 \mathrm{kHz}$; 16-bit resolution). (d) Hour 7 in woodland savannah with re-growing grass in Gamgam (sampling frequency: $44.1 \mathrm{kHz}$; 16-bit resolution). 


\subsubsection{Ambient noise level}

Mean $( \pm \mathrm{SD})$ ambient noise level was lower in Gamgam $(42.43 \pm 3.03 \mathrm{~dB})$ than in Kwano (46.19 $\pm 6.12 \mathrm{~dB}$; exact Mann-Whitney U-test: $\left.\mathrm{N}_{1}=\mathrm{N}_{2}=36, \mathrm{U}=432.5, \mathrm{p}=0.015\right)$. Throughout the dry season, mean $( \pm \mathrm{SD})$ ambient noise level did not differ significantly between forest $(41.89 \pm 3.14 \mathrm{~dB})$ and woodland savannah $(42.98 \pm 2.90 \mathrm{~dB})$ in Gamgam (exact Wilcoxon T-test: $\mathrm{N}=18, \mathrm{~T}=46.5, \mathrm{p}=0.163$ ). In Kwano however, forest (mean $\pm \mathrm{SD}$ : $43.86 \pm 4.75 \mathrm{~dB}$ ) was significantly quieter than woodland savannah (mean $\pm \mathrm{SD}$ : $48.51 \pm 6.56 \mathrm{~dB}$; exact Wilcoxon T-test: $\mathrm{N}=18, \mathrm{~T}=23.0, \mathrm{p}=0.005)$. However, these statements varied over the dry season. In Gamgam, forest was significantly quieter than woodland savannah when grass was high, but the difference was not significant when grass was low or re-growing. In Kwano, forest was significantly quieter than woodland savannah when grass was low and re-growing, but the difference was not significant when grass was high (Table 3.1 and Figure 3.4).

Table 3.1: Mean $( \pm \mathrm{SD})$ ambient noise level $[\mathrm{dB}]$ recorded $1 \mathrm{~m}$ above ground in forest and woodland savannah in both study sites in the three sessions. The last line for each study site give the T-value (p-value in brackets) for the exact 2-tailed Wilcoxon T-tests $(\mathrm{N}=24)$ used to compare ambient noise level between habitat types.

\begin{tabular}{l|l|ccc}
\multicolumn{2}{l|}{} & High grass & Low grass & Re-growing grass \\
\hline \multirow{3}{*}{ Gamgam } & Forest & $41.01 \pm 2.86$ & $40.99 \pm 3.75$ & $43.67 \pm 3.72$ \\
& Woodland savannah & $43.03 \pm 2.43$ & $41.48 \pm 3.36$ & $44.42 \pm 2.98$ \\
& T-value (p-value) & $49.0(0.003)$ & $122.0(0.435)$ & $128.5(0.550)$ \\
& & & & $48.36 \pm 5.76$ \\
\multirow{3}{*}{ Kwano } & Forest & $42.56 \pm 2.07$ & $40.67 \pm 2.24$ & $54.47 \pm 7.29$ \\
& Woodland savannah & $43.56 \pm 3.17$ & $47.49 \pm 4.59$ & $39.0(0.001)$
\end{tabular}




\section{Gamgam}
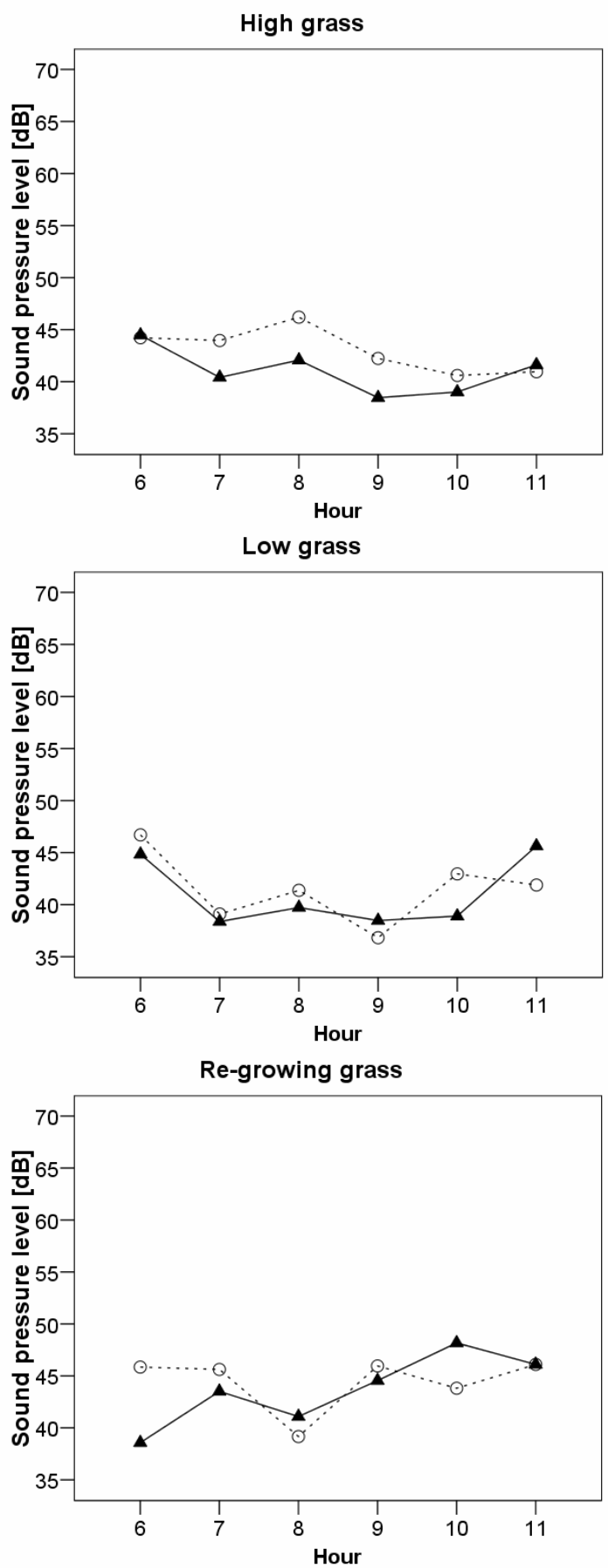

Kwano
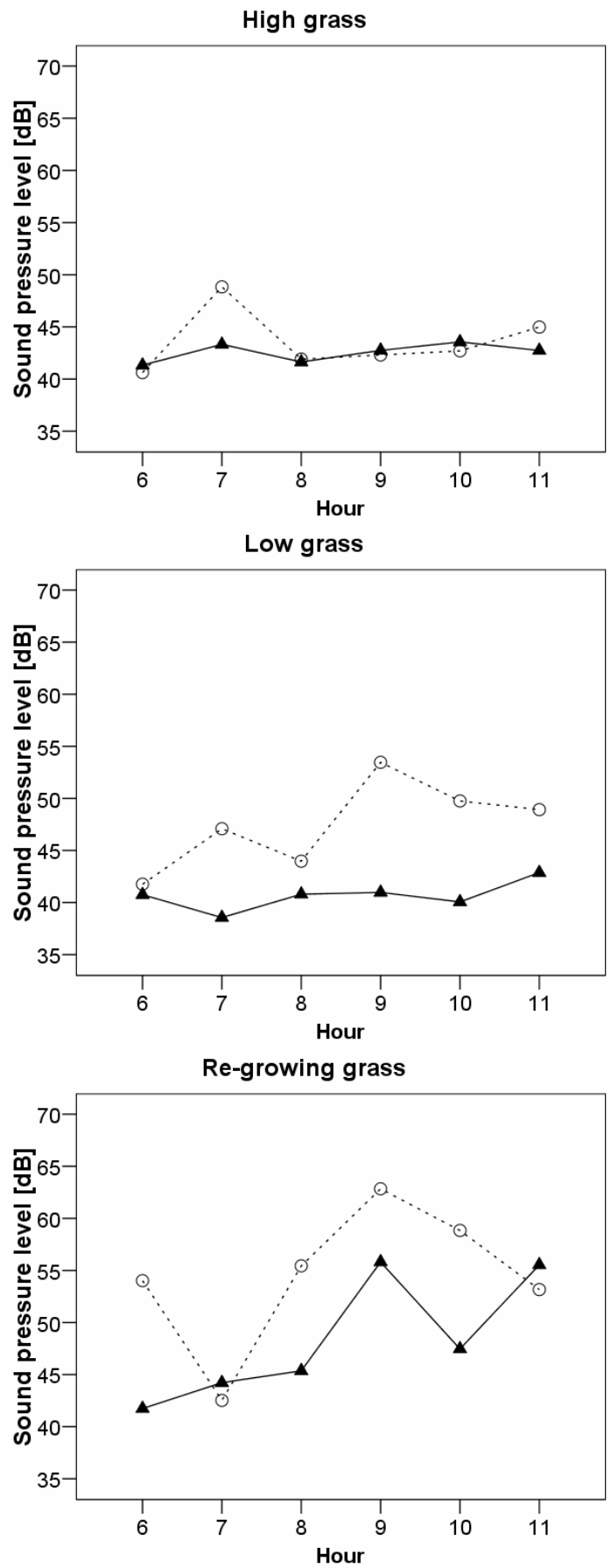

Figure 3.4: Mean ambient noise level $[\mathrm{dB}]$ over the whole morning in forest (triangles) and woodland savannah (circles) in Gamgam (left) and Kwano (right). Recordings were done $1 \mathrm{~m}$ above ground, in the four compass directions. 
The quietest mornings with the lowest mean $( \pm \mathrm{SD})$ ambient noise levels in the two habitat types together occurred in Gamgam when grass was low $(41.23 \pm 3.53 \mathrm{~dB})$, whereas it occurred when grass was high in Kwano $(43.06 \pm 2.70 \mathrm{~dB})$. The loudest mornings occurred in both study sites when grass began to re-grow (mean $\pm \mathrm{SD}$ : Gamgam: $44.04 \pm 3.35 \mathrm{~dB}$; Kwano: $51.41 \pm 7.20 \mathrm{~dB}$ ). The high ambient noise level at this time was mostly due to insects.

In Gamgam, mean ambient noise level decreased slightly over the morning hours when grass was high in forest and woodland savannah and when grass was low in woodland savannah but not in forest. In contrast, it increased over the morning hours when grass was low in forest and when grass began to re-grow in forest and in woodland savannah (Table 3.2). In Kwano, mean ambient noise level increased slightly over the morning hours in both habitat types in the three sessions except in woodland savannah with re-growing grass (Table 3.2).

Table 3.2: Mean ( $\pm \mathrm{SD}$ ) ambient noise level $[\mathrm{dB}]$ recorded $1 \mathrm{~m}$ above ground early (hour 6$)$ and late (hour 11) morning in forest and in woodland savannah in both study sites in the three sessions during the dry season.

\begin{tabular}{l|l|cc|cc|cc}
\multicolumn{2}{l|}{} & \multicolumn{2}{|c|}{ High grass } & \multicolumn{2}{c|}{ Low grass } & \multicolumn{2}{c}{ Re-growing grass } \\
\multicolumn{2}{l|}{} & Hour 6 & Hour 11 & Hour 6 & Hour 11 & Hour 6 & Hour 11 \\
\hline \multirow{3}{*}{ Gamgam } & Forest & $44.48 \pm 1.54$ & $41.63 \pm 4.80$ & $44.84 \pm 1.98$ & $45.64 \pm 4.35$ & $38.58 \pm 2.56$ & $46.10 \pm 1.04$ \\
& $\begin{array}{l}\text { Woodland } \\
\text { savannah }\end{array}$ & $44.23 \pm 1.58$ & $40.96 \pm 1.42$ & $46.70 \pm 1.32$ & $41.89 \pm 1.07$ & $45.85 \pm 2.56$ & $46.10 \pm 1.48$ \\
\hline \multirow{2}{*}{ Kwano } & $\begin{array}{l}\text { Forest } \\
\text { Woodland } \\
\text { savannah }\end{array}$ & $41.34 \pm 3.10$ & $42.74 \pm 1.36$ & $40.75 \pm 4.11$ & $42.88 \pm 1.78$ & $41.74 \pm 0.88$ & $55.54 \pm 2.17$ \\
& $40.63 \pm 1.44$ & $44.99 \pm 0.20$ & $41.76 \pm 3.25$ & $48.93 \pm 3.25$ & $54.03 \pm 7.50$ & $53.16 \pm 0.90$
\end{tabular}

\subsubsection{Energy distribution}

Mean $( \pm \mathrm{SD})$ over sessions of mean peak frequency was much lower in Gamgam (forest: $1176 \pm 1631 \mathrm{~Hz}$; woodland savannah: $1244 \pm 1342 \mathrm{~Hz}$ ) than in Kwano (forest: $2289 \pm 1900 \mathrm{~Hz}$; woodland savannah: $3352 \pm 2997 \mathrm{~Hz}$ ).

Only two examples of morning variations in frequencies at which $25 \%$ (dfa1), $50 \%$ (dfa2) and $75 \%$ (dfa3) of the global energy were reached are described here. In Kwano forest, when grass was high, dfa1, dfa2, and dfa3 were dispersed far from one another (for instance, dfa3 - dfa1 $=5222 \pm 1216 \mathrm{~Hz} \quad[$ mean $\pm \mathrm{SD}]$ at hour 11; Figure 3.5a). Dfa1 was low (mean \pm SD over the morning: $1719 \pm 1335 \mathrm{~Hz}$ ) and peak frequency lay within the frequency range of bird vocalisations $(52 \mathrm{~Hz}-5214 \mathrm{~Hz}$; Figure $3.5 b)$.

When grass was low in Kwano woodland savannah, dfa1, dfa2, and dfa3 were close to one another (e.g., dfa3 - dfa1 $=2042 \pm 440 \mathrm{~Hz}$ [mean $\pm \mathrm{SD}$ ] at hour 6; Figure 3.5c). In the first half of the morning, at least $75 \%$ of the global energy were concentrated in frequencies 
lower than $4000 \mathrm{~Hz}$ and peak frequency was low (46 Hz - $776 \mathrm{~Hz})$. From hour 9, $25 \%$ of the global energy were reached only around $8 \mathrm{kHz}$ and $75 \%$ of the global energy were reached around $10 \mathrm{kHz}$. The mean ( $\pm \mathrm{SD}$, between hour 9 and hour 11) peak frequency was as high as $5936 \pm 2716 \mathrm{~Hz}$ (Figure 3.5d).

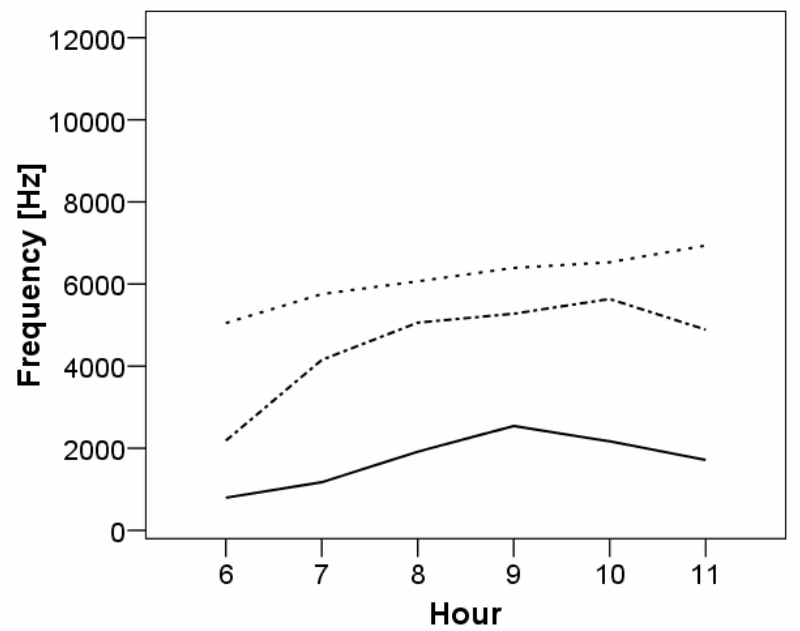

c.

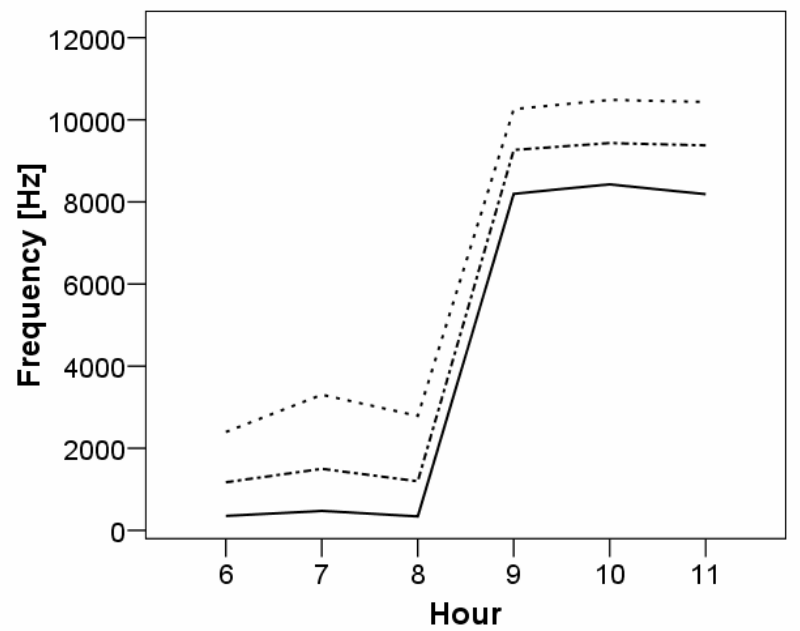

b.

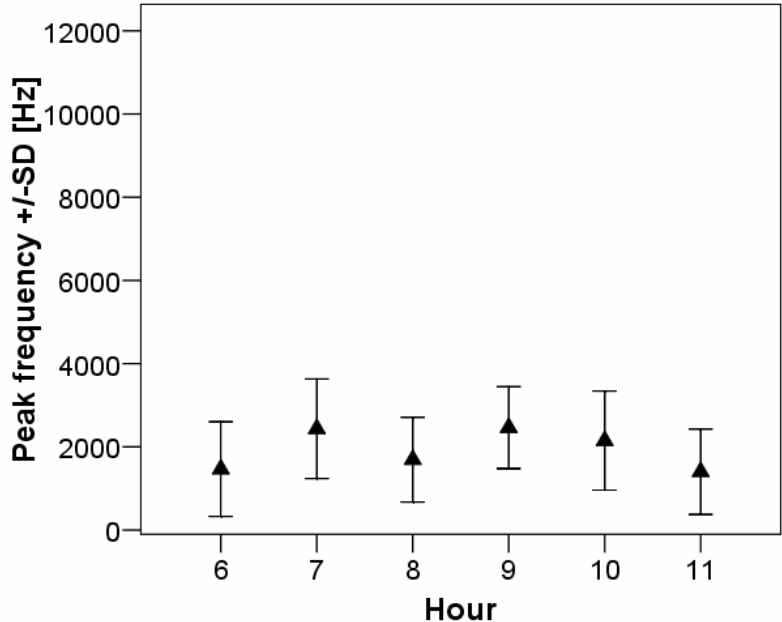

d.

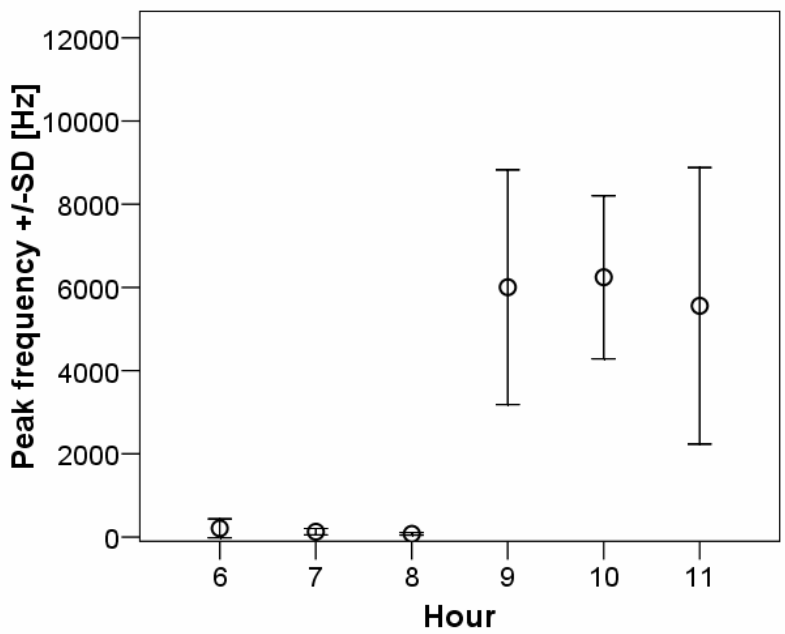

Figure 3.5: Mean frequencies at which $25 \%(\mathrm{dfa} 1-$ ), $50 \%(\mathrm{dfa} 2-\cdot-\cdot)$ and $75 \%(\mathrm{dfa} 3---)$ of the global energy were reached in ambient noise in Kwano (a) in forest when grass was high and (c) in woodland savannah when grass was low. Mean $( \pm \mathrm{SD})$ peak frequency of ambient noise in Kwano (b) in forest when grass was high and (d) in woodland savannah when grass was low. Recordings were done $1 \mathrm{~m}$ above ground, in the four compass directions. 


\subsubsection{Test of attenuation}

A general pattern could be detected in attenuation profiles in both habitat types in the three sessions at both sites (Figure 3.6). Frequencies of $50 \mathrm{~Hz}$ and $400 \mathrm{~Hz}$ were attenuated very strongly from $5 \mathrm{~m}$ already. Amplitude for the pure tone of $50 \mathrm{~Hz}$ could not be measured reliably from $12.5 \mathrm{~m}$. Frequencies of $100 \mathrm{~Hz}$ and $200 \mathrm{~Hz}$ underwent little attenuation, as did frequencies of $1000 \mathrm{~Hz}, 2000 \mathrm{~Hz}$ and $4000 \mathrm{~Hz}$. These had a minimal attenuation up to $50 \mathrm{~m}$. Frequencies of $600 \mathrm{~Hz}$ and $800 \mathrm{~Hz}$ also underwent little attenuation, but did not propagate as well as the previous ones. Frequencies higher or equal to $6000 \mathrm{~Hz}$ were rapidly attenuated over distances larger than $12.5 \mathrm{~m}$ (attenuation profiles diverged strongly from the parallel to the attenuation at $5 \mathrm{~m}$ and $12.5 \mathrm{~m})$. The highest frequencies tested $(14000 \mathrm{~Hz}$ and $16000 \mathrm{~Hz})$ underwent the highest measurable attenuation over $50 \mathrm{~m}$.

However, within the above-cited general pattern, some variations occurred between habitat types and across sessions. Attenuation of high frequencies was accentuated in habitat types showing an obstructed visibility (i.e., forest in all three sessions and woodland savannah with high grass; Figure 3.6a) in comparison to more open habitat types (i.e., woodland savannah with low and re-growing grass and to a lesser extent with naturally evolving grass). In these last open habitat types, attenuation profiles measured from $12.5 \mathrm{~m}$ to $50 \mathrm{~m}$ stayed close to the parallel to the attenuation at $5 \mathrm{~m}$ (Figure 3.6b). For instance, the highest frequencies $(14000 \mathrm{~Hz}$ and $16000 \mathrm{~Hz})$ were attenuated similarly to frequencies between $6000 \mathrm{~Hz}$ and $10000 \mathrm{~Hz}$. 
a.

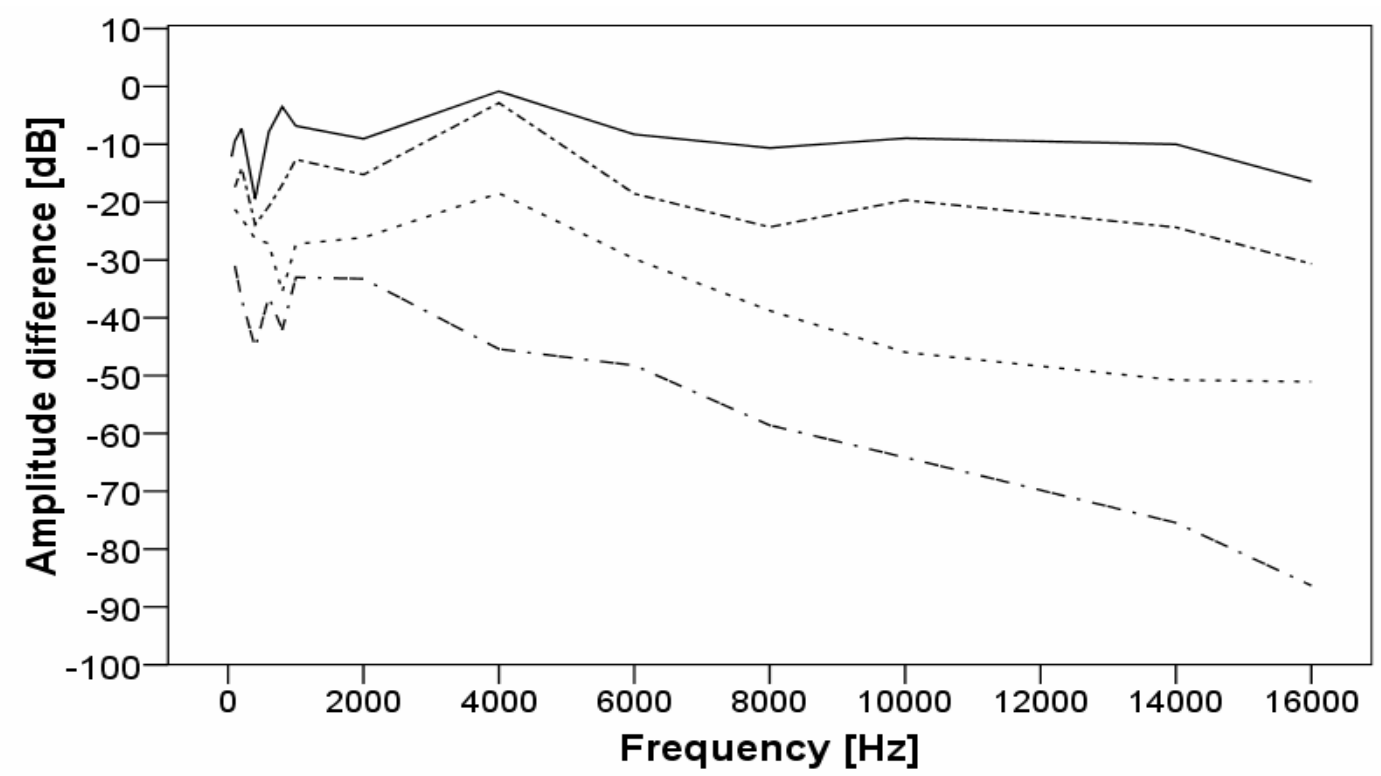

b.

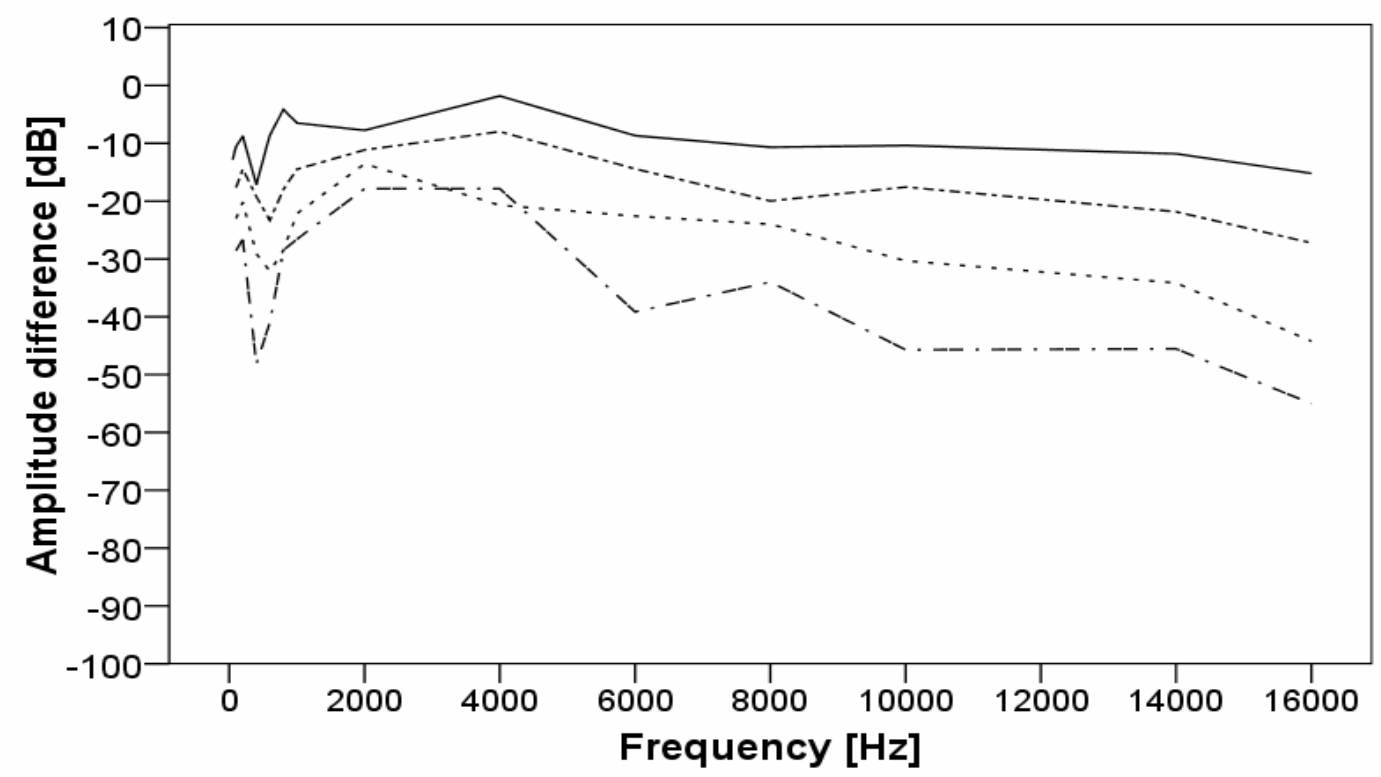

Figure 3.6: Attenuation profiles in Gamgam when grass was re-growing. (a) Important attenuation of high frequencies in forest. (b) Limited attenuation of high frequencies in woodland savannah. Synthetic signal broadcasts and re-recordings were done $1 \mathrm{~m}$ above ground. Recording distances: $(-): 5 \mathrm{~m} ;(-\cdot-\cdot): 12.5 \mathrm{~m}$; $(--): 25 \mathrm{~m} ;(--\cdot--\cdot): 50 \mathrm{~m}$. 


\subsection{DISCUSSION}

Methods presented in this chapter provide a good overview of the structural (tree density, visual vegetation density) and acoustic (ambient noise, attenuation) properties of an environment, while still being easy to put into practice. Results of the present example of application highlighted important variations within habitat types, related to grass height for instance. A combination of the various environmental aspects characterised is necessary. Indeed, local variations might be blurred by a too gross habitat description. Each environmental aspect will now be discussed in more details.

\subsubsection{Tree density}

The point-quarter sampling method is rapid and relatively simple to use. Forest had a higher tree density than woodland savannah in both study sites. However, overlaps between forest and woodland savannah in Kwano and between woodland savannah in Gamgam and forest in Kwano (Figure 3.1) reveal that tree densities can be similar between two gross habitat types. Measurements of tree density should then be combined with other measures which describe more precisely other structural aspects of the environment, such as undergrowth or grass cover. A more accurate structural description might then be provided by a combination with measurements of visual vegetation density.

Estimations to distinguish different habitats might also be refined by considering tree density in different size categories. This might be helpful to distinguish some variants of one habitat category (e.g., more or less wooded variants of woodland savannah) and to explain differences within habitat types. For instance, the lower tree density in woodland savannah in Gamgam than in Kwano seems to be due to a very low density of small trees $(5 \leq \mathrm{DBH}<10 \mathrm{~cm})$ in Gamgam (see Appendix 3$)$. Indeed, the proximity of this site to a village leads to a strong pressure from humans looking for firewood, for which small trees are preferred. Additionally, a more intense burning gives access to a larger surface of woodland savannah for these people. 


\subsubsection{Visual vegetation density}

Estimations of visual vegetation density complement tree density measurements by providing a characterisation of undergrowth and/or grass cover. These features might undergo large seasonal variations, as it is the case for grass height within woodland savannah in the present example of application. For such a finer characterisation, standardisation of the simple method presented is crucial. For instance, the height from which the picture is taken can play a determinant role since the picture might differ importantly if it is taken above or within grass. The present example of application nevertheless lacks a comparison with pictures taken from $0.5 \mathrm{~m}$ or $1 \mathrm{~m}$ above ground to illustrate this point.

This method provides a quantification of an intuitive fact (forest and woodland savannah with high grass were most visually obstructed, whereas woodland savannah with low and re-growing grass offered the best visibility). This might be useful to build visibility categories (to take into account habitat patchiness) and to compare home ranges between groups or populations. This classification of habitat types also corresponds well to attenuation conditions (see hereafter) and might represent a kind of proxy for them.

Woodland savannah tended to be less visually obstructed in Gamgam than in Kwano. This might be due to a more intensive burning around the village and agricultural surfaces of Gashaka. People might also cut more trees for firewood in woodland savannah near the village, a hypothesis that might also be drawn from tree density (see above). This suggests that the history of an environment and anthropological influences should be considered to understand some variations within and between habitat categories.

\subsubsection{Ambient noise}

\subsubsection{Sources of ambient noise}

Examining spectrograms leads to a description of frequency ranges encompassing ambient noise amplitude. Studying energy distribution provides a more formal analysis. The advantage of descriptive results is to give biological meaning to formal analyses. Main sources of ambient noise (insects, birds, and wind) and their particular frequency ranges were identified, and results confirmed those of previous studies (Ryan \& Brenowitz 1985; Waser \& Brown 1986; Slabbekoorn 2004; Schneider et al. 2008). The most favourable frequency range for acoustic communication was between $1000 \mathrm{~Hz}$ (above non-biotic noise) and $4000 \mathrm{~Hz}$ 
(under continuous insect noise). Discontinuous noise from birds occurred as the main source of ambient noise in this frequency range.

Knowing frequency ranges disturbed by ambient noise allows to predict disturbances on acoustic signals and could explain frequency ranges used by some species in acoustic communication. For instance, loud calls of Kloss gibbons, Mentawai macaques, Mentawai leaf monkeys and pig-tailed langurs on Siberut Island, Indonesia, fell into the most favourable frequency range with the lowest continuous ambient noise (Schneider et al 2008). This frequency range was similar to the one found in this chapter $(1000-4000 \mathrm{~Hz})$. However, insects can be very loud and they can therefore disturb sound reception, even if they are not within the frequency range of the acoustic signals considered. The recipient might encounter difficulties (because of saturation in his auditory organs) in distinguishing the signal of interest from loud noise of insects if this one occupies frequency ranges just around the signal of interest. This confirms the statement of Ryan and Brenowitz (1985) about ambient noise as a non-negligible constraint in long-range communication. In contrast, Waser and Brown (1986) suggested that, over long distances, attenuation differences between habitats outweigh ambient noise differences. This sounds less realistic according to personal observations.

\subsubsection{Ambient noise level}

Recordings of ambient noise level complement data on visibility and attenuation rates. The flexibility of the method described (i.e., directions of recording, recording height and distribution over the day) allows to characterise precisely local variations and to track seasonal and daily changes. Forest tended to be quieter than woodland savannah. Even in comparison to a primary rainforest on Siberut Island, Indonesia (Schneider et al. 2008), forest in Nigeria was slightly quieter. This general pattern (forest tending to be quieter than woodland savannah, in contrast to what Waser and Brown [1986] found) varied over sessions and across morning hours. Therefore, the habitat type which was the most favourable to acoustic communication -in the domain of ambient noise level only- varied also. However, distributing recordings over season and day allows to study whether animals follow these variations for spontaneous calls. For instance, ambient noise level seemed to be more favourable early morning than late morning. When sound pressure level was high, the contribution of insects to ambient noise was important. Insects might therefore represent the most important disturbance for acoustic communication (see above). Of course, other environmental features than ambient noise level such as attenuation rate might interplay and influence the vocal behaviour of animals. 
Limited morning variations in ambient noise levels found in the present application contrast with clear patterns found in some previous studies. For instance, in deciduous temperate forest, ambient noise was lowest just before dawn and highest in mid-morning (Dabelsteen \& Mathevon 2002). On tropical forest floor, levels were low early morning but peaked after dawn (bird choruses), then dropped again in late morning and raised a second peak in the evening (insects; Waser \& Waser 1977). This time-dependence was mainly related to call rhythms of animals and to daily variations of wind speed. The restricted variations in Gamgam and Kwano might stem from differences in insect and bird communities, which might have "postponed" calling times, in comparison to previous studies. However, limited variations in ambient noise levels over the morning hours in forest (except at the end of the dry season) were not exceptional. Schneider and her co-workers (2008) also found only little variations between 6:00 and 15:00 in ambient noise level in a primary rainforest on Siberut Island, Indonesia.

\subsubsection{Energy distribution}

Recordings used to evaluate energy distribution can be conducted at the same time as those used to measure ambient noise level. This allows to save time and to track seasonal and daily variations. Quantifying energy distribution in ambient noise complements the visual inspection of spectrograms by a more formal analysis. For instance, in Kwano forest, when the grass was high (Figure 3.5a and b), the large frequency range of ambient noise suggested that all sources (wind, birds and insects) contributed together to ambient noise (which was quiet according to the corresponding sound pressure level). The low dfal and peak frequency suggested that mostly birds and wind (and few insects) contributed to ambient noise. Insects might have contributed slightly more in the late morning as dfa1, dfa2 and dfa3 rose. When grass was low in Kwano woodland savannah (Figure 3.5c and d), the concentration of energy in low frequencies and the low peak frequency in the first half of the morning suggested that few insects contributed to ambient noise, which was probably due to wind and some birds with low frequency vocalisations. The increase in dfa1 and dfa2 and the high mean peak frequency suggested that from hour 9 insects began to call and contributed largely to ambient noise, other sources (wind, birds) becoming negligible. These data should allow to predict which acoustic signals are suited or not according to season, habitat type or time of day. Animals should favour signalling whenever the peak frequency of ambient noise is not included in the frequency range of their spontaneous signals. For instance, frequency ranges of primate loud calls were not overlapping with frequency ranges of insects, but with those of 
the discontinuous calls of birds and frogs in a primary rainforest on Siberut Island, Indonesia (Schneider et al. 2008).

Energy was concentrated in higher frequencies late morning in Gamgam when grass was re-growing and in Kwano throughout the dry season. This phenomenon is attributable to an increase in the proportion of insect noise (insects were quieter in the first two hours of the morning than later), but also to a rise in insect noise frequency, which might be correlated with an increase in temperature (Slabbekoorn 2004). Besides, measurements of peak frequency confirmed the impression (from visual inspection of spectrograms) that noise from wind was equivalent between the two sites and that birds represented a more prominent source of ambient noise in Gamgam (lower peak frequency), while insects were a stronger source of ambient noise in Kwano (higher peak frequency).

\subsubsection{Test of attenuation}

Frequency-dependent sound attenuation reflects precise acoustic features of each habitat type, but the importance of habitat patchiness should not be neglected. Determining sound attenuation should allow to predict adaptations in acoustic signals (see Chapter 2). In the present application, attenuation tests lead to a classification similar to the one based on visual vegetation density, with forest and woodland savannah with high grass more similar to each other than to woodland savannah with low and re-growing grass. It confirms that attenuation becomes more important with increasing vegetation density, as for instance between open habitats and forests (Waser \& Brown 1986), and, within forests, between open and dense forests (Padgham 2004), between canopy and ground in moist evergreen forests (Waser \& Waser 1977), and between "before" and "after" foliation (Blumenrath \& Dabelsteen 2004). However, a reduced but non-negligible attenuation in an open habitat such as woodland savannah stems from deviation on turbulent air (Morton 1975; Piercy et al. 1977; Richards \& Wiley 1980; Fricke 1984; Waser \& Brown 1986; Fotheringham \& Ratcliffe 1995; Naguib \& Wiley 2001; Naguib 2003) and / or ground effect (Fricke 1984). Attenuation might be even more important in open places than in wooded ones in some tropical habitats (Waser \& Brown 1986) and in some temperate places (Aylor 1972).

Very low $(50 \mathrm{~Hz})$ and very high frequencies $(14000 \mathrm{~Hz}$ and $16000 \mathrm{~Hz})$ were most rapidly attenuated, and more so in habitats which were cluttered by vegetation, such as forest and woodland savannah with high grass. These habitats have therefore the highest attenuation potentials. Frequencies of 100, 200, 800, 1000 and $2000 \mathrm{~Hz}$ were attenuated similarly in all 
habitat types. Above $2000 \mathrm{~Hz}$, frequencies were more strongly attenuated in forest (except in the session with high grass in Gamgam) than in other habitats. These results were similar to those of Chapuis (1971), but he did not examine the influence of grass height.

Considering low frequencies, the important attenuation of $50 \mathrm{~Hz}$ in all habitat types might be due to the ground effect which enhances attenuation -through refraction- of low frequencies from sources closer than $1 \mathrm{~m}$ to the ground (Aylor 1972; Waser \& Waser 1977; Cosens \& Falls 1984; Price et al. 1988; Nelson 2003). The strong attenuation of $400 \mathrm{~Hz}$ was more surprising, but might also be due to ground effect. In this case, recording height might play an important role and its standardisation is therefore necessary. The broadcasting height chosen in the present application ( $1 \mathrm{~m}$ above ground) was realistic for relatively large mammals living on ground. However, broadcasting sine waves from other heights (lower or higher) might lead to different attenuation profiles (Marten \& Marler 1977; Marten et al. 1977).

For given conditions (e.g., porous soil and low elevation), frequencies between $1000 \mathrm{~Hz}$ and $3000 \mathrm{~Hz}$ present a minimal excess attenuation because ground effect is reduced (Marten \& Marler 1977; Cosens \& Falls 1984). The good transmission of tones of $1000 \mathrm{~Hz}$ and $2000 \mathrm{~Hz}$-and to some extent of $100 \mathrm{~Hz}$ and $200 \mathrm{~Hz}$ (see optimum transmission frequencies in particular habitat types hereafter)- in the present study might be explained by such "ground effect windows" (Morton 1975; Forrest 1994). These ones were found to concern slightly lower frequencies in forests $(1585-2500 \mathrm{~Hz}$, Morton 1975) than in more open habitats (Marten et al. 1977; temperate habitat: 1000 - 3000 Hz, Marten \& Marler 1977; Florida scrub habitat: 2500 - $4500 \mathrm{~Hz}$, Nelson 2003). However, in moist evergreen forest, in rainforest and in an open habitat (in comparison to a primary / old secondary forest), the optimum transmission frequencies were very low: $125 \mathrm{~Hz}$ (Waser \& Waser 1977), 100 $200 \mathrm{~Hz}$ (Waser \& Brown 1984), and $315-500 \mathrm{~Hz}$ (Sugiura et al. 2006), respectively. The good transmission of tones of $100 \mathrm{~Hz}$ and $200 \mathrm{~Hz}$ found in this application could then reflect optimum transmission frequencies of the habitats under study.

\subsection{CONCLUSIONS}

The present chapter provides methods to characterise structural and acoustic properties of an environment. Combining these methods to inter-relate their results is necessary to obtain a sufficient level of precision, while still taking into account potential local variations. For instance, forest appeared to be the most favourable environment for acoustic communication 
when considering ambient noise level, but not when considering attenuation. Therefore, if only one aspect is characterised, a possible interplay with other environmental features should not be forgotten in studies about environmental influences on acoustic signals.

The methods provided here suggest standards for habitat characterisation and present several non-negligible advantages. Their convenience and relative simplicity allow a reliable and rapid characterisation of structural and acoustic aspects of an environment without investing too much in highly specialised material and without any need of particular skills in botanic. Even if these methods do not allow any further investigation in processes of sound attenuation, they confirmed previous studies on habitat characterisation, which might attest their reliability. They allow a comparison between habitat types, and can also be adapted to particular niches relevant to the species under study. The home ranges of two populations of olive baboons (in Uganda and in Nigeria) are actually characterised with visual vegetation density, which appears to reflect attenuation conditions (at least in Nigeria). Chapters 4 and 5 investigate whether baboons of both populations modify usage and acoustic structure of their contact calls according to the environment in which they vocalise. 


\title{
Chapter 4
}

\section{From open to closed habitat - Flexibility in grunt structure and usage in wild female olive baboons}

\begin{abstract}
Sounds propagate differently and visibility varies according to the habitat type (Chapter 3). Animals are therefore expected to adjust the acoustic structure and usage of their vocal signals to these environmental characteristics (Chapter 2). In the present chapter, the influence of the habitat on the vocal behaviour of olive baboons is evaluated within two populations: one living in Budongo Forest Reserve, Uganda, and the other one in Gashaka-Gumti National Park, Nigeria. This chapter focuses on grunts, which are quiet contact calls used for shortdistance communication (see Chapter 1). It examines whether female baboons modify the acoustic structure of their grunts and their rate of grunting when they wander between closed and open habitat types. As an adaptation to environmental conditions, baboons are expected to utter calls with a longer duration, a lower fundamental frequency and / or energy concentrated in lower frequencies in a closed habitat than in an open one. Baboons should also grunt more frequently in a closed habitat. Analyses showed that grunts uttered in non-interactive contexts were significantly longer in forest than in open habitat. In addition, baboons from Uganda uttered non-interactive grunts at a significantly higher rate in forest than in open habitat, as expected. However, these effects were weaker in grunts given in interactive contexts. Variations in grass height led to different adjustment patterns in grunt acoustic structure and grunt rate. These results suggest that other factors such as the balance in usage of vocal and visual signals in specific contexts and the proximity between group members might override the effect of the environment. They might also explain variability between populations in intra-individual flexibility in grunt rate.
\end{abstract}

This chapter was written in collaboration with Charlotte Rahn, who collected data in Uganda. Julia Fischer and Kurt Hammerschmidt revised some parts of an earlier version. 


\subsection{INTRODUCTION}

Understanding design and usage of vocal signals in relation to the environment is important to understand the structure of a communication system as a whole. Thus, in the last decades, much attention has been given to the potential influence of the habitat on vocal communication in animals. Studies analysing phenomena associated with acoustic signal propagation (e.g., absorption, reverberation; reviewed in Piercy et al. 1977, Wiley \& Richards 1978) and evaluating acoustic properties of different habitat types (e.g., Marten \& Marler 1997; Marten et al. 1977; Waser \& Brown 1986) provided an important basis for investigations on the effects of the environment on vocal signals. Since habitat type affects sound transmission, animals are expected to adapt their vocal behaviour to structural and acoustic properties of the environment they inhabit. Various species were found to use different strategies to improve signal transmission in their habitat. These strategies concerning both usage of vocal signals and their acoustic structure are reviewed in Chapter 2. Most studies were conducted in birds, but some investigated similar questions in primates (e.g., Waser \& Waser 1977; Waser \& Brown 1984, 1986; Brown \& Waser 1988; Masters 1991; Brown et al. 1995; Mitani \& Stuht 1998; Rendall et al. 2000; de la Torre \& Snowdon 2002; Sugiura et al. 2006; Koda et al. 2008; Schneider et al. 2008).

Nearly all studies testing the "acoustic adaptation hypothesis" conducted so far were exclusively dedicated to comparisons between species or populations living in different habitat types. In both cases, ruling out the influence of a genetic factor is difficult and only few studies managed to do so. They either simply documented genetic proximity between the populations compared or introduced phylogenetic relatedness between species or populations as a factor in their analyses (birds: e.g., Blumstein \& Turner 2005, Nicholls \& Goldizen 2006; primates: Sugiura et al. 2006; reviewed in Chapter 2). One possibility to avoid the interplay of several other factors (e.g., phylogenetic relatedness, social system) is to compare vocalisations of the same individuals ranging in different environments, as suggested in Chapter 2. This has been done once in wild chacma baboons to investigate the relationship between bark rate and habitat type (Rendall et al. 2000), but never in wild primates to investigate the effect of visibility and transmission properties of the habitat on the acoustic structure of vocal signals. Therefore, in this chapter, the vocal behaviour of the same individuals is compared between different habitat types. This allows to test whether olive baboons ranging in both closed and open habitats adjust the acoustic structure of their vocalisations and their call rate to the environment in which they currently range. The influence of the gross habitat type (forest vs. 
open habitat), as well as the influence of seasonal variations in grass height within one habitat type (woodland savannah with high grass vs. woodland savannah with low and re-growing grass) on acoustic structure and call rate were tested. Data collected from the Nigerian population of olive baboons (see Chapter 1) were combined with data from another population of olive baboons in Uganda. The focus of this chapter was set on contact calls used for short-distance communication (i.e., grunts; see Chapter 1). So far, studies on environmental influences on vocal communication in primates have focused on calls used over long distances, while little -if anything- is known about environmental effects on vocalisations used over shorter distances, like grunts. When baboons utter these calls, they are often about $10 \mathrm{~m}$ apart from one another (E. Ey, C. Rahn, pers. obs.). This is much closer than when they utter clear barks for instance, but still makes an influence of the environment on usage and structure of these calls conceivable. Grunts are likely to present environmental adjustments since they are aimed at reaching recipients without degradation. Indeed, the relative proximity between callers and recipients (usually $0-15 \mathrm{~m}$; pers. obs.) does not justify any ranging process for this call type. Besides, predation risk is very low in the study troops (E. Ey, C. Rahn, pers. obs.) and grunts are therefore not constrained in their propagation distance (see General introduction).

According to some mechanisms of sound transmission, baboons should show at least one of the following adaptations to the habitat to optimise transmission distance and/or detectability of their grunts: longer duration (Nemeth et al. 2006), lower fundamental frequency (e.g., Chapuis 1971) or energy concentrated in lower frequencies (e.g., Morton 1975) in a closed habitat (forest, woodland savannah with high grass) than in an open habitat (grassland, woodland savannah with low or re-growing grass). Additionally, grunt rate should be higher in closed than in open habitats. This would increase the localisability and likelihood of detection in closed habitats (Waser \& Waser 1977; Macpherson \& Middlebrooks 2000). Environmental influences on both structure and usage were expected to be limited in contexts in which animals are close to one another (i.e., in direct social interactions), in comparison to when they are more distant (Koda et al. 2008). The effect of the environment was also expected to be more important on grunt rate than on acoustic structure. As a matter of fact, in contrast to usage of vocal signals which is known to be flexible, call production is considered to be largely innate (see General introduction). 


\subsection{MATERIAL AND METHODS}

\subsubsection{Study sites}

\subsubsection{Uganda}

In Uganda, data were collected by C. Rahn in one troop of olive baboons -Sonso troop- living in Budongo Forest Reserve, close to the river Sonso and to the Budongo Conservation Field Station. Budongo Forest is an isolated forest block located on the edge of the western Rift Valley, western Uganda (137' - $2^{\circ} 00^{\prime} \mathrm{N}, 31^{\circ} 22^{\prime}-31^{\circ} 46^{\prime} \mathrm{E}$ ), at an average altitude of $1100 \mathrm{~m}$ (Eggeling 1947). The reserve covers an area of $825 \mathrm{~km}^{2}$ of forest and grassland, of which $437 \mathrm{~km}^{2}$ are covered with moist semi-deciduous tropical forest (Howard et al. 1997). Budongo Forest has been commercially managed for timber on a sustainable yield basis since the mid 1920s (Howard 1991; Paterson 1991) and therefore is currently a mosaic of vegetation types (Plumptre et al. 1994). The distribution of rainfall throughout the year is bimodal, with most rain falling between March and May and between September and November and a dry season lasting from December to February. Mean annual rainfall is $1780-1900 \mathrm{~mm}$. On average, annual minimum and maximum temperatures are $17-21^{\circ} \mathrm{C}$ and $27-29^{\circ} \mathrm{C}$, respectively.

\subsubsection{Nigeria}

In Nigeria, I focused on the two troops of olive baboons -Gamgam and Kwano troopsalready described in Chapter 1. As a brief reminder, the home range of Gamgam troop, along Gamgam river near the village of Gashaka, is mostly covered by southern Guinea savannah (hereafter woodland savannah), with narrow bands of riverine forest along seasonal streams. Kwano troop ranged around Kwano field station, mostly in lowland rainforest, but it also visited patches of woodland savannah. Both home ranges underwent burning, leading to large variations in grass height in woodland savannah. For a complete description of both home ranges, see the characterisation of Gamgam and Kwano study sites in Chapter 3.

\subsubsection{Habitat types}

When testing the effect of gross habitat categories, two habitat types were distinguished (data collected at the border between the two habitat types or in other vegetation formation were discarded). The closed habitat type, hereafter "forest", presented a low visibility; it encompassed forested areas in both Uganda and Nigeria. The open habitat type, 
hereafter "open habitat", had a better visibility and included grassland in Uganda and woodland savannah with low and re-growing grass in Nigeria. Visibilities in the respective habitat types were similar between Uganda and Nigeria (Appendix 4). A more precise study of structural and acoustic properties of the habitat types in Nigeria revealed that visibility reflects sound attenuation, which is higher in forest than in an open habitat type like woodland savannah with low and re-growing grass (Chapter 3 ) and presumably grassland.

When examining the influence of grass height within woodland savannah in Nigeria, the closed habitat type was represented by woodland savannah with high grass and the open habitat type by woodland savannah with low and re-growing grass (low grass hereafter). Woodland savannah with high grass, as a closed habitat type, had a low visibility and a high attenuation of high frequencies (Chapter 3).

\subsection{2 $\underline{\text { Animals }}$}

All three troops were habituated to human presence, in Uganda shortly after the beginning of the study and in Nigeria since 2000. Adults and subadults were recorded from a $2-12 \mathrm{~m}$ distance. The size and composition of the troops varied slightly over the observation period: 18 - 19 individuals in Sonso troop, 20 - 21 individuals in Gamgam troop, and 24 - 29 individuals in Kwano troop. In the present chapter, the focus was set on adult and subadult females (see Table 4.1 for sample sizes). There were too few adult males from which data were available (one in Sonso and Gamgam troops and three in Kwano troop).

A phylogenetic analysis based on mitochondrial DNA indicates that the Ugandan and Nigerian populations belong to two deeply separated clades or lineages of olive baboons. The genetic distance between these two clades would justify to classify them as two different subspecies of olive baboons (Zinner et al. 2008). The two Nigerian troops share the same haplotypes, suggesting that they comprise members of the same matrilines (Zinner et al., in press). 
Table 4.1: Composition of each data set used for analyses on grunt acoustic structure and grunt rate. Numbers in brackets indicate the number of grunts included in the acoustic analyses.

\begin{tabular}{l|cc|c} 
& \multicolumn{2}{|c|}{ Nigeria } & Uganda \\
& Gamgam & Kwano & Sonso \\
\hline Structure: non-interactive, forest / open & \multicolumn{2}{|c}{8 females (86) } & 6 females (89) \\
Structure: interactive, forest / open & 13 females (168) & \\
Structure: non-interactive, high / low grass & \multicolumn{2}{|c|}{6 females (84) } & \\
Structure: interactive, high / low grass & \multicolumn{2}{|c}{8 females (96) } & \\
Grunt rate: forest / open & 5 females & 10 females & 7 females \\
Grunt rate: high / low grass & 5 females & 10 females &
\end{tabular}

\subsubsection{Data collection}

Data were collected over 2.5 months around the transition between the rainy and the dry seasons (Apr 07 - Jul 07) in Uganda and over 10 months distributed over two dry seasons (Nov 05 - Dec 05, Feb 06 - May 06, and Nov 06 - Apr 07) in Nigeria. In total, Sonso troop was observed $173.5 \mathrm{~h}$, Gamgam troop $370 \mathrm{~h}$, and Kwano troop $455 \mathrm{~h}$. Among this time, $74.4 \mathrm{~h}$ in Sonso troop, $156.5 \mathrm{~h}$ in Gamgam troop, and $208.5 \mathrm{~h}$ in Kwano troop were spent collecting data (behavioural data and audio-recordings) on the focal females. In Uganda, animals were observed in $15 \mathrm{~min}$ focal follows distributed over the day between 8:00 and 17:00 with sampling points (scans) every $3 \mathrm{~min}$. In Nigeria, one focal animal was followed each day from 6:00 to 12:00, with sampling points (scans) for behavioural data every 15 min. For each sampling point, habitat type, activity of the focal animal and number of individuals within $10 \mathrm{~m}$ of the focal animal were recorded. All behaviours were classified into two context categories identical to the ones in Chapter 1: "non-interactive" and "interactive". As a brief reminder, non-interactive activities included foraging, resting and travelling. In these contexts, the animals were not directly interacting with any other group member. Interactive activities encompassed all direct social interactions, such as friendly approaches toward an individual, infant handling, grooming/being groomed, embracing/being embraced, presenting/receiving a presentation, mounting/being mounted, and aggressing/being aggressed. These two context categories did not only consider that grunt structure or grunt rate might differ between different behavioural contexts (Owren et al. 1997; see also Chapter 1). They also reflected two different initial situations for vocal communication, namely that animals are either close to each other when calling (interactive situations) or rather distanced and spread over a larger area (non-interactive situations; see Chapter 1). 
Grunts were recorded with a digital solid-state recorder (Marantz PMD660; Marantz, Japan) and a Sennheiser directional microphone (K6 power module and ME66 recording head with a Rycote softie windscreen; Sennheiser Electronic KG, Germany) with a sampling frequency of $44.1 \mathrm{kHz}, 16-$ bit resolution and in mono. Whenever possible, grunts from other individuals than the focal animal were also recorded. For each vocalisation, information about time, caller identity, context, and habitat type was stored and recording distance was estimated.

For each focal animal, a general grunt rate was calculated by counting all grunts (i.e., the units in bouts) uttered by this individual and dividing this number by the total observation time for this individual. This general grunt rate was calculated over the time spent in forest and open habitat (not in woodland savannah with high grass) to obtain comparable data between Uganda and Nigeria. A grunt rate for grunts given in non-interactive activities and a grunt rate for grunts given in interactive activities were also calculated for each focal animal in each habitat type. Two slightly different methods were used between Uganda and Nigeria because of differences in the data collection protocol (see above). The number of grunts given in each context category was divided by the time spent in each habitat type. In Uganda, grunt rates were calculated over 3-min intervals, assuming that animals spent the entire interval in the same habitat type and that all grunts within one interval were given in the same context. In contrast, in Nigeria the precise duration of time spent in each habitat type and the precise context of each grunt were known. This inter-population difference in calculations does not affect comparisons of grunt rates between habitat types, because these were made within each population separately.

\subsubsection{Acoustic analyses}

In total, 892 grunts were collected in Sonso troop, 4698 in Gamgam troop, and 2962 in Kwano troop, from all focal females. Among these calls, grunts recorded from 3 - $12 \mathrm{~m}$ were selected, as calls might be distorted over longer distances (Fischer et al. 2002). After additional exclusion of calls disturbed by background noise and calls not given in closed (forest, woodland savannah with high grass) or open (grassland, woodland savannah with low grass) habitat types, 601, 1077 and 636 grunts of good quality remained for the acoustic analyses in Sonso, Gamgam and Kwano troops, respectively. Among these recordings, 2 - 14 grunts given in non-interactive activities per female per habitat type were further selected from 6 adult females in Uganda. In Nigeria, 2 - 10 grunts per animal per habitat type (same 
number of grunts in forest and open habitat) were selected from 8 adult females in noninteractive activities and from 13 adult females in interactive activities. To examine the effect of grass height within woodland savannah, 2 - 10 grunts per animal per grass height (same number of grunts in high and low grass) were selected from 6 females in non-interactive activities and 8 females in interactive activities (Table 4.1). These last selections provided data sets with an even distribution of calls between habitat types and between callers.

The software Avisoft SASLab Pro Recorder 4.3 (R. Specht; Berlin, Germany) was used to select high-quality grunts. Sampling frequency was lowered to $5512.5 \mathrm{~Hz}$ to obtain a higher frequency resolution in the frequency range of grunts. Duration was measured on the first harmonic on spectrograms calculated by Avisoft (sampling frequency: $5512.5 \mathrm{~Hz}$; FFTlength: 1024 points; Hamming window; overlap: $98.43 \%$; time resolution: $2.9 \mathrm{~ms}$; frequency resolution: $10.8 \mathrm{~Hz}$ ), using its standard cursor function. Binary spectrograms were then saved and files were exported into the software LMA 2005 developed by K. Hammerschmidt (Schrader \& Hammerschmidt 1997). The harmonic cursor tool was used to calculate mean fundamental frequency and mean peak frequency for each grunt (start and end amplitude thresholds: $10 \%$; cut-off frequency: set under fundamental frequency and as far as possible above background noise [i.e., according to the call at $35 \mathrm{~Hz}, 42 \mathrm{~Hz}, 50 \mathrm{~Hz}, 58 \mathrm{~Hz}$, and $70 \mathrm{~Hz}$ ]; noise factor: 1.5 ; repetition factor: 1 ; amplitude calculation mode: general; no filter; contour threshold: 0.2; F0 modulation limit: $30 \%$; F0 range for IM: 0.5). The distribution of values for each acoustic variable was checked afterwards to identify potential outliers. For these, measurements were then either re-done or calls were excluded from the analyses.

\subsubsection{Statistical analyses}

A linear mixed model with population (Uganda / Nigeria), habitat type (forest / open habitat) and the interaction population $\times$ habitat type as fixed factors was used to examine variations between forest and open habitat in duration, mean fundamental frequency and mean peak frequency of grunts given in non-interactive activities from both populations together. For grunts given in interactive activities (for which only data from Nigeria were available), a linear mixed model with habitat type (forest/open habitat) as a fixed factor was used. For grunts given in non-interactive and interactive activities in high and low grass, only data from Nigeria were available. A linear mixed model with grass height (high grass / low grass) as a fixed factor was used in each context category separately to examine the influence of grass height on duration, mean fundamental frequency and mean peak frequency. In all linear 
mixed models described above, caller identity was considered as a random factor and $\mathrm{p}$-values were corrected with a Step-up Hochberg correction for multiple testing since the same model was used in the three acoustic variables tested (Westfall \& Young 1993).

General grunt rates were compared between troops and between populations by using exact 2-tailed Mann-Whitney U-tests because sample sizes were small (see Mundry \& Fischer 1998). To investigate the influence of the habitat on grunt rate in non-interactive and interactive activities separately, a linear mixed model with habitat type (forest / open habitat) as a fixed factor was used in each population separately. To test the influence of grass height within woodland savannah on grunt rate in interactive and non-interactive activities, a linear mixed model with grass height (high / low) as a fixed factor was used. In these two linear mixed models, the identity of the focal animal was considered as a random factor. The proportion of scans in interactive activities was compared between woodland savannah with high grass and woodland savannah with low grass using an exact 2-tailed Wilcoxon T-test.

All statistical analyses were done with the software SPSS 15.0 for Windows. The significance level was set at 0.05 .

\subsection{RESULTS}

\subsubsection{Grunt acoustic structure}

\subsubsection{Forest vs. open habitat}

Grunts given in non-interactive activities were significantly longer in forest than in open habitat (Table 4.2a), while the effects of the population (duration: $F=2.85$, corrected $p=0.825$; mean fundamental frequency: $F=7.15$, corrected $p=0.160$; mean peak frequency: $F=0.59$, corrected $p=0.846$ ) and of the interaction population $\times$ habitat type (duration: $F=0.22$, corrected $p=0.846 ;$ mean fundamental frequency: $F=1.06$, corrected $\mathrm{p}=0.846$; mean peak frequency: $\mathrm{F}=0.28$, corrected $\mathrm{p}=0.846$ ) were not significant. In contrast, in grunts given in interactive activities, none of the acoustic variables varied significantly with habitat type (Table $4.2 \mathrm{~b}$ ). There was only a tendency for interactive grunts to be longer in forest than in open habitat. 
Table 4.2: Mean and standard deviation of duration, mean fundamental frequency (F0 mean) and mean peak frequency (Pf mean) of grunts given in forest and open habitat in (a) non-interactive and (b) interactive activities. The last line gives F-values and corrected p-values (in brackets) for the factor habitat type in the linear mixed model.

a.

\begin{tabular}{l|ccc} 
& Duration [ms] & F0 mean [Hz] & Pf mean [Hz] \\
\hline Forest (91 grunts) & $190 \pm 31$ & $84 \pm 14$ & $307 \pm 120$ \\
Open (84 grunts) & $171 \pm 33$ & $84 \pm 16$ & $290 \pm 130$ \\
Habitat type effect & $16.16(<0.001)$ & $0.04(0.846)$ & $0.77(0.846)$
\end{tabular}

b.

\begin{tabular}{l|ccc} 
& Duration [ms] & F0 mean [Hz] & Pf mean [Hz] \\
\hline Forest (84 grunts) & $181 \pm 36$ & $79 \pm 14$ & $284 \pm 101$ \\
Open (84 grunts) & $172 \pm 29$ & $79 \pm 12$ & $295 \pm 103$ \\
Habitat type effect & $4.92(0.084)$ & $0.01(0.903)$ & $0.50(0.903)$
\end{tabular}

\subsubsection{High grass vs. low grass in woodland savannah}

Only data from Nigeria were available. In non-interactive activities, there was a significant influence of grass height on peak frequency, but not on any other acoustic variable (Table 4.3a). Non-interactive grunts presented a lower peak frequency in high grass than in low grass. In interactive activities, grass height did not have any significant effect on any of the three acoustic variables tested (Table $4.3 \mathrm{~b}$ ). There was only a tendency for interactive grunts to be shorter in high grass than in low grass.

Table 4.3: Mean and standard deviation of duration, mean fundamental frequency (F0 mean) and mean peak frequency (Pf mean) of grunts given in woodland savannah with high grass and woodland savannah with low grass in (a) non-interactive and (b) interactive activities. The last line gives F-values and corrected p-values (in brackets) for the factor grass height in the linear mixed model.

a.

\begin{tabular}{l|ccc} 
& Duration [ms] & F0 mean [Hz] & Pf mean [Hz] \\
\hline High grass (42 grunts) & $167 \pm 35$ & $71 \pm 16$ & $220 \pm 92$ \\
Low grass (42 grunts) & $172 \pm 28$ & $70 \pm 16$ & $285 \pm 106$ \\
Grass height effect & $0.66(0.420)$ & $1.48(0.420)$ & $13.47(0.001)$ \\
b. & Duration [ms] & F0 mean [Hz] & Pf mean [Hz] \\
\hline High grass (48 grunts) & $173 \pm 24$ & $74 \pm 12$ & $311 \pm 107$ \\
Low grass (48 grunts) & $182 \pm 27$ & $75 \pm 11$ & $330 \pm 70$ \\
Grass height effect & $3.85(0.090)$ & $0.46(0.391)$ & $1.09(0.391)$
\end{tabular}




\subsubsection{Grunt rate}

Mean $( \pm \mathrm{SD})$ grunt rates did not differ significantly between females of the two Nigerian troops (Gamgam: $0.31 \pm 0.18$ grunts / min, Kwano: $0.19 \pm 0.10$ grunts / min; exact Mann-Whitney U-test: $\mathrm{N}_{1}=5, \mathrm{~N}_{2}=10, \mathrm{U}=14, \mathrm{p}=0.206$ ), but differed significantly between females of the Ugandan troop $(0.49 \pm 0.21$ grunts / min $)$ and those of both Nigerian troops together $\left(0.23 \pm 0.14\right.$ grunts / $\mathrm{min}$; exact Mann-Whitney U-test: $\mathrm{N}_{1}=7, \mathrm{~N}_{2}=15, \mathrm{U}=14$, $\mathrm{p}=0.005)$. Therefore, females of the two Nigerian troops were considered together for the following analyses.

\subsubsection{Forest vs. open habitat}

In Uganda, the emission rate of grunts given in non-interactive activities varied significantly with habitat type (linear mixed model: $\mathrm{F}=11.55, \mathrm{p}=0.015$ ). Baboons uttered non-interactive grunts at a significantly higher rate in forest than in open habitat. In contrast, the rate at which interactive grunt were uttered did not vary significantly with habitat type (linear mixed model: $\mathrm{F}=0.71, \mathrm{p}=0.430$; Figure 4.1a).

In Nigeria, neither the emission rate of non-interactive grunts (linear mixed model: $F=1.03, p=0.327$ ) nor the emission rate of interactive grunts (linear mixed model: $F=0.13$, $\mathrm{p}=0.723$ ) varied significantly with habitat type (Figure $4.1 \mathrm{~b}$ ). 
a.

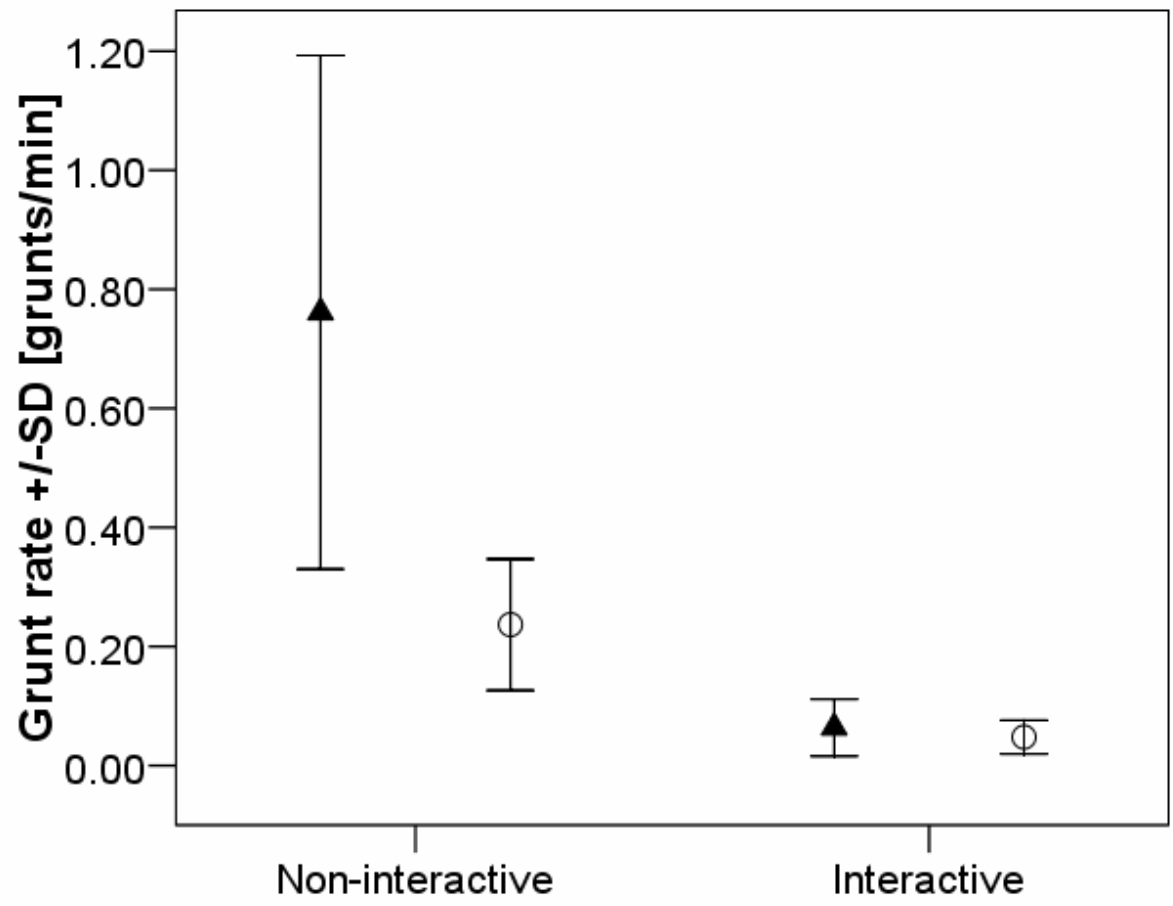

b.

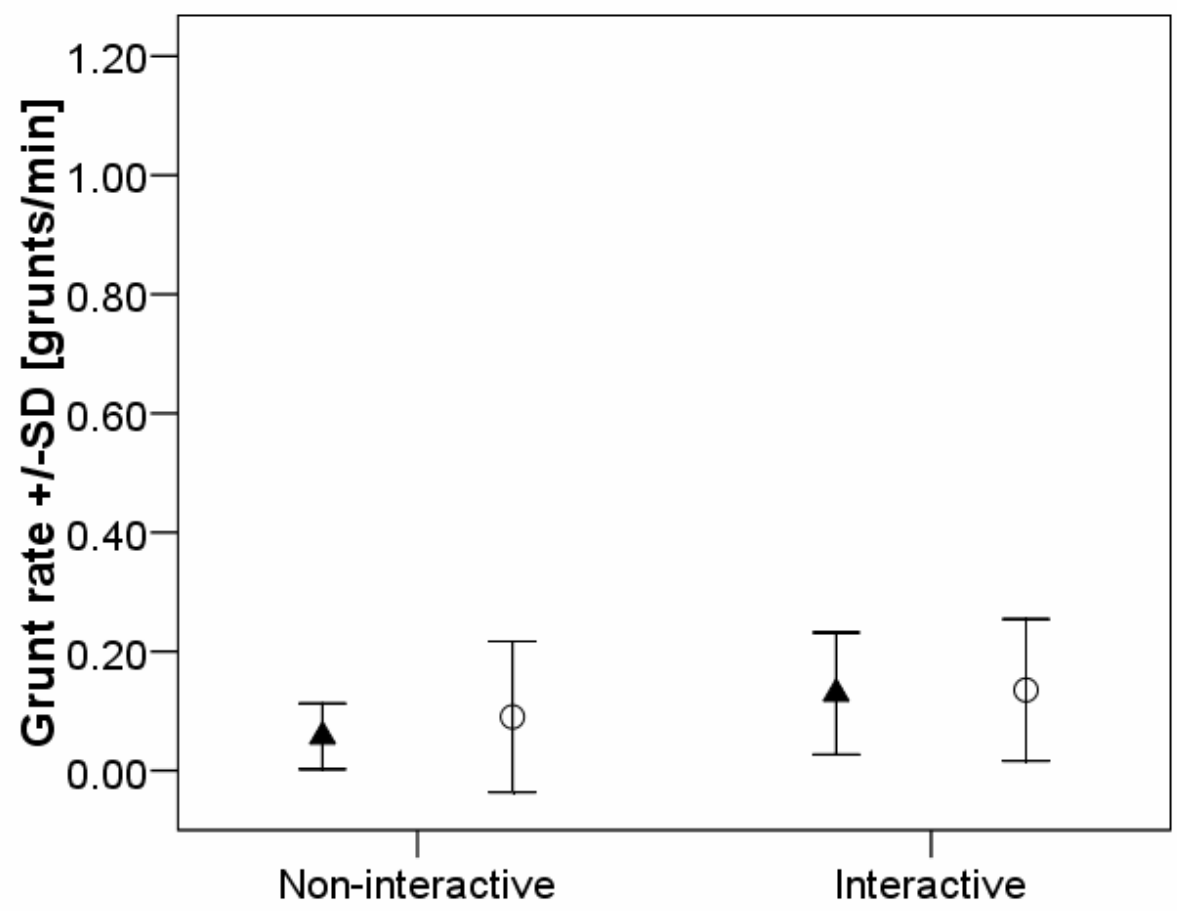

Figure 4.1: Mean and standard deviation of the individual grunt rate in "non-interactive" and "interactive" contexts in forest (black triangles) and in open habitat (circles) in (a) Uganda and (b) Nigeria. 


\subsubsection{High grass vs. low grass in woodland savannah}

The emission rate of non-interactive grunts did not vary with grass height (linear mixed model: $\mathrm{F}<0.01, \mathrm{p}=0.990$; Figure 4.2). In contrast, female baboons uttered interactive grunts at a significantly higher rate in woodland savannah with low grass than in woodland savannah with high grass (linear mixed model: $F=7.94, p=0.009$ ). Grunt rate for interactive grunts was extremely low in high grass (Figure 4.2), but this did not seem to be due to the fact that female baboons spent less time socialising in high grass than in low grass. Indeed, the mean $( \pm \mathrm{SD})$ proportion of scans in interactive activities was similar in high grass $(11.7 \pm 27.6 \%$ of scans in woodland savannah with high grass) and in low grass $(10.9 \pm 9.3 \%$ of scans in woodland savannah with low grass; exact Wilcoxon T-test: $\mathrm{N}=13$, $\mathrm{T}=20.5, \mathrm{p}=0.290)$.

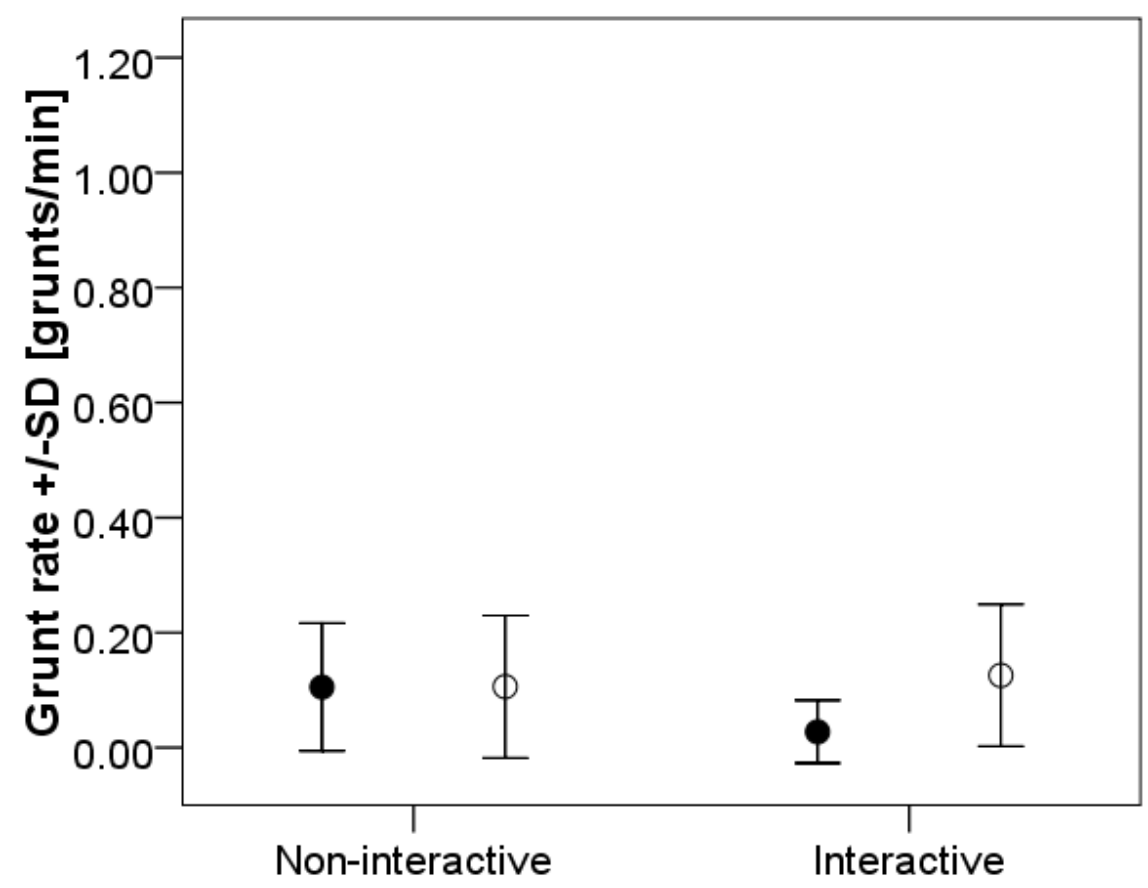

Figure 4.2: Mean and standard deviation of the individual grunt rate in "non-interactive" and "interactive" contexts in woodland savannah with high grass (black circles) and in woodland savannah with low grass (i.e., open habitat; white circles) in Nigeria. 


\subsection{DISCUSSION}

This chapter investigated whether wild female olive baboons from two populations adjusted the acoustic structure of their grunts and their grunt rate to the habitat in which they vocalised. Baboons from both populations lengthened their grunts in forest, and baboons from Nigeria lowered grunt peak frequency in high grass, in comparison to open habitat with low grass. Concerning usage, baboons from Uganda grunted at a higher rate in forest than in open habitat, as predicted. These habitat-related variations occurred only in non-interactive contexts, confirming predictions. In contrast, baboons from Nigeria adjusted their grunt rate only to grass height and contrary to expectations.

\subsubsection{Grunt acoustic structure}

\subsubsection{Forest vs. open habitat}

Duration was found to be a flexible acoustic variable over a short-term, since individuals of both baboon populations uttered longer non-interactive grunts in forest than in open habitat. In contrast, fundamental frequency and peak frequency appeared to vary relatively independently of habitat type. Duration is one of the acoustic variables in which no consistent environmental influences could be found, in birds at least (Chapter 2). This might nonetheless be explained by the fact that Chapter 2 reviewed mostly studies comparing the vocal behaviour of species or populations and not of individuals ranging in different habitat types. Variations in duration were small (only a few milliseconds). However, this represents approximately $10 \%$ of the total grunt duration, and might then be relatively non-negligible and biologically salient. Fischer and colleagues (1998) found that small variations of some tens of Hertz (in first and third dominant frequency bands, frequency range and amplitude distribution) in calls of Barbary macaques between two populations are detected by these primates. Flexibility in call duration was evoked earlier by Sutton and colleagues (1973) who successfully trained three juvenile rhesus macaques to lengthen their coo calls. More recent studies in primates also highlighted some intra-individual flexibility over a short-term in this acoustic variable. For instance, captive common marmosets increase not only amplitude of their twitter calls with increasing ambient noise level but also duration (Brumm et al. 2004), as do cotton-top tamarins in their combination long calls (Egnor \& Hauser 2006). Individuals of this species also shorten their combination long calls over experiments to avoid overlap with artificial ambient noise (Egnor et al. 2007). 
These findings may suggest that, in the present study, grunts were longer in forest than in open habitat because animals reacted to a higher ambient noise level in forest. However, this hypothesis is unlikely because, at least in Nigeria, ambient noise tended to be quieter in forest than in open habitat (Chapter 3). In addition, linking longer calls in forest to an increased arousal (Rendall 2003) because of low visibility can also be excluded since the other acoustic variables (fundamental frequency and peak frequency) did not vary at the same time. It seems more plausible to assume that lengthening calls in a closed habitat with many reverberating surfaces might improve call propagation. Amplitude of reverberated sound waves might be added to direct waves when both wave types overlap. This should allow sounds to carry further (Nemeth et al. 2006). Baboons might also lengthen their grunts to counteract the larger loss of energy due to propagation through vegetation in forest in comparison to open habitat. This would facilitate detection by recipients, which is known as the temporal summation phenomenon (e.g., Clack 1965; Dooling 1979; Dooling \& Searcy 1985; Brown \& Maloney 1986; Klump \& Maier 1990). Indeed, for short signals like grunts, when stimulus duration increases, the amplitude level required for signal detection decreases (see Brown \& Maloney 1986). Finally, lengthening calls might also increase their localisability (Macpherson \& Middlebrooks 2000; Brumm et al. 2004), since spatial detection through the binaural system is more efficient on longer sounds (Macpherson \& Middlebrooks 2000). Neither fundamental frequency nor peak frequency varied between gross habitat types. These acoustic variables might be more strongly related to other factors such as individuality, arousal and specific contexts of vocal utterance (see Rendall 2003; Meise 2008).

Analysis conducted on grunts given in interactive activities in Nigeria revealed the importance of the context in which vocalisations are uttered. Grunts given in direct social interactions did not show significant variation in duration (or fundamental or peak frequency) according to habitat type. This reduced (since there is still a trend in duration) adaptation in grunt structure might be explained by the fact that individuals are closer to one another in interactive situations than when no direct social interaction occurs in both habitat types (Chapter 1). Over such short distances, visibility between animals as well as transmission features are similar between forest and open habitat. In these cases, the visual communication channel can complement the auditory one (Marler 1977) and vocal signals travel through the environment only for a short distance until they reach the receiver. Adapting grunt structure is therefore not necessary. In fact, it would be useful only in situations where individuals are communicating with rather distanced group members because, only under these circumstances, environmental characteristics become important in sound propagation. 
Therefore, significant differences of grunt structure between habitats in interactive contexts would have even been surprising. Future studies should examine whether proximity between caller and recipient also weakens the effect of habitat type in interactive grunts of baboons from other populations, for instance Uganda. Furthermore, it should also be tested whether proximity between caller and recipients influences the adjustment to the environment in grunt production within non-interactive contexts. Not enough data were available in the present thesis for such finer analyses. For instance, the magnitude of the variation in duration should be compared between situations in which individuals are quite far from one another $(10-$ $15 \mathrm{~m}$ ) and situations in which they are closer (within $2 \mathrm{~m}$ ) within a non-interactive context like resting. The influence of inter-individual distances on call structure was already suggested in isolation calls of squirrel monkeys (Masataka \& Symmes 1986) and coo calls of Japanese macaques (Sugiura 2007) for instance (see General introduction).

It remains an open question whether the ability of adjusting grunt acoustic structure to the environment is learned by baboons during ontogenesis or if a basic neuronal circuit might be responsible for a prolongation of grunts in forest, for instance as a reaction to hearing degraded and quieter calls of group members. This further step could be achieved if calls from immature individuals were collected. A possible ontogenetic development of the ability to adapt calls to the environment could then be tracked. It would indicate how much experience is needed for a baboon to adjust grunt duration to environmental conditions. Grunts are particularly suited for such a study since they are given from one week of age already (Chalmers 1980). Ideally, the same individuals should be recorded throughout their development in both habitat types, but this could be done only over a very long-term. Otherwise, grunt acoustic structure should be compared between habitat types within age classes constituted of different individuals, like in the study of Ey and colleagues (2007b) on age- and sex-related variations in chacma baboon clear barks.

\subsubsection{High grass vs. low grass in woodland savannah}

As in the comparison of grunt acoustic structure between forest and open habitat, there was no significant influence of grass height on any of the three acoustic variables tested in grunts given in interactive situations. The tendency contrary to expectation in duration might be due to a restricted sample size, with few calls per individual. The absence of any significant effect might be explained by the fact that animals are close to one another during direct social interactions. Therefore, adjusting grunt acoustic structure to environmental features is not worth (see above). In contrast, in non-interactive situations, the pattern of 
adjustment differed from the one found when comparing grunt acoustic structure between gross habitat types. Non-interactive grunts had a lower peak frequency in high grass than in low grass and there was no effect of grass height on duration. Animals might concentrate energy in low frequencies because these ones propagate further through vegetation than high frequencies (e.g., Morton 1975; Marten \& Marler 1977; Marten et al. 1977; Forrest 1994; see also Chapter 3). The effect on peak frequency cannot be simply due to the grass which was between the caller and the microphone and which might attenuate high frequencies, even over small recording distances (Mathevon et al. 1996). If this were the case, a similar effect would have been found in both interactive and non-interactive contexts, which did not occur.

Variations in acoustic structure seem to be specific to very precise conditions, as already suggested in Chapter 2. As soon as one factor varies, the effect of the habitat might also be modified. The present adjustment might differ from the one used between forest and open habitat because grass might affect more the immediate environment and might lead to a more closed environment immediately around the caller (i.e., more cluttered over a shorter distance than in forest; pers. obs.). Varying peak frequency might be more useful than varying duration in such environmental conditions. This remains to be tested by broadcasting synthetic grunt-like signals with controlled duration and peak frequency and re-recording them from various distances in the different habitat types. This complex pattern of variations with precise environmental features should nevertheless be considered carefully since shortterm variations in peak frequency have not been found in primate calls so far. To draw some reliable conclusions, further investigations with an increased data set in both interactive and non-interactive contexts are needed. No data concerning the influence of grass height (or any other seasonally changing structural feature of the environment) on vocal signals have been found in primates, especially when the same individuals are recorded in different environmental conditions.

\subsubsection{Grunt rate}

\subsubsection{Forest vs. open habitat}

Ugandan baboons adjusted their grunt rate to their environment, and therefore to visibility and sound transmission conditions. They grunted at a higher rate in forest than in open habitat, probably to increase detection likelihood and localisability (Waser \& Waser 1977; Macpherson \& Middlebrooks 2000). However, they did so only for non-interactive grunts. Grunt structure was already found to be adjusted to the local habitat in non-interactive 
contexts but not in interactive ones (see above). Similar reasons could justify that a higher grunt rate in forest may be of interest mostly in non-interactive contexts. In these situations (e.g., foraging, travelling), individuals are much more scattered than in direct social interactions (Chapter 1 for Nigerian baboons). More frequent vocal exchanges between group members might then serve to counteract higher attenuation of sound waves and reduction of information from the visual channel in forest, in comparison to open habitat (Waser \& Waser 1977). This should help to keep contact between group members. This finding parallels results of Koda and colleagues (2008) who found that Japanese macaques from a population ranging in a visibly obstructed habitat utter coo calls (contact calls used over short distances) at a higher rate than those from a population living in a more open area.

In contrast to Uganda, grunt rates in Nigeria were similar between the two habitat types in both non-interactive and interactive contexts. This inter-population difference, even if it was not expected, is in accordance with the hypothesis of an important flexibility in call usage (Seyfarth \& Cheney 1997; see also Chapter 1). It demonstrates that olive baboons have the ability to adapt their grunt rate to the environment (Uganda) but do not necessarily use it (Nigeria). To what extent the phylogenetic distance between these two populations explains this difference is unknown. A potential explanation for the absence of variation with habitat type in grunt rate of Nigerian baboons is that this population has a very low general grunt rate, twice as low as baboons from Uganda (see also discussion in Chapter 1 for data on both males and females). Grunt rates in interactive contexts were similar between Uganda and Nigeria. In contrast, in non-interactive contexts, Nigerian baboons grunted very rarely compared to Ugandan baboons (inter-population divergence in the calculation of grunt rates in each context prevented any statistical test). The inter-population difference in general grunt rate cannot be explained by slight differences in the way of calculating grunt rates. The general grunt rate was based on the total observation time, independently of habitat type, for the interpopulation comparison (see material and methods). General usage of grunts might therefore differ between the Ugandan and the Nigerian populations. The former might rely more on vocal communication, while the later might rely more on visual communication, even when animals are not directly interacting. These differences in contact call usage might stem from the interplay of various factors, such as the precise contexts of calling (i.e., in which contexts baboons utter non-interactive grunts) and group spread (i.e., the proximity between group members), which can override the effect of the environment. For instance, Nigerian baboons might have a lower grunt rate than Ugandan ones because they might form more cohesive (less dispersed) troops and therefore rely more on the visual channel. Further investigations 
involving other baboon populations are needed to clarify how these various factors interplay to regulate grunt usage. Precise contexts of calling and group spread (for instance the number of individuals within $10 \mathrm{~m}$ of the focal animal) should be systematically recorded to obtain measures comparable to those from Nigeria.

\subsubsection{High grass vs. low grass in woodland savannah}

Nigerian female baboons gave interactive grunts at different rates between high grass and low grass in woodland savannah. Contrary to expectations, interactive grunt rate was significantly higher in low grass (open habitat type) than in high grass (closed habitat type). This variation cannot be explained by the fact that baboon grunted less often in high grass to avoid attracting predator attention. If this were the case, the same variation would have occurred in non-interactive contexts. This divergence from the preceding results in which grunt rate adjustment was neither found in interactive contexts nor in Nigerian baboons is intriguing. The grunt rate difference between high and low grass is due to the surprisingly low interactive grunt rate in high grass. Why female baboons are grunting so rarely in social interactions in high grass is for the moment unknown, since it cannot be explained by the fact that baboons spend less time in interactive activities in high grass than in low grass. Future investigations should increase sample size, because the present data are based on short time periods spent in high grass. Besides, the social interactions and the corresponding vocal behaviour occurring in high and low grass should be compared more precisely. For such a comparison, the exact amount of time spent in direct social interactions should be measured and grunts and their precise contexts of occurrence (e.g., groom, approach, infant handling) should be recorded.

To sum up, results revealed a certain amount of intra-individual flexibility in vocal production and call usage of baboons in response to the environment. Nevertheless, flexibility in response to more subtle environmental changes such as grass height variations remains unclear. Future studies should investigate a potential ontogenetic development of plasticity in grunt duration, and concentrate on factors potentially explaining inter-population variability in grunt usage between baboon populations. 


\title{
Chapter 5
}

\section{Usage of long-distance communication calls - Limited adjustment to environmental conditions in wild olive baboons}

\begin{abstract}
Environmental characteristics such as ambient noise level and vegetation density lead to a masking effect on vocal signals and attenuation during their propagation (Chapter 3 ). Animals are therefore expected to utter their vocalisations in a way reducing effects of these phenomena. Such strategies should be most useful in calls used over long distances, since these vocalisations are intended to carry far while being in contact with the environment for a long time. The present chapter examines whether olive baboons ranging in both forest and open habitat (woodland savannah in Nigeria and grassland in Uganda) adjust usage of calls used for long-distance communication to the habitat type and ambient noise level. Two types of vocalisations are examined: clear barks (i.e., loud barks given by individuals isolated from the rest of the group), and loud calls (i.e., bouts of two-phased loud barks given by adult males only; see Chapter 1). Both call types are expected to be given at a higher rate in forest where visibility is lower and sound propagation more difficult than in the more open habitat type. Loud calls are expected to be uttered preferentially when background noise level is the lowest, that is, early morning (Chapter 3 ). Contrary to predictions, bark rate did not vary significantly according to habitat type and therefore to visibility conditions. In contrast, a tendency emerged for adult males to utter loud calls in the first half of the morning when background noise level is the lowest, as expected. Overall, these results suggest that emission rate of clear barks is mostly constrained by the situation in which these vocalisations are given (including predation risk in the area), which seems to override the effect of the environment, while the timing of loud call utterances appears more flexible.
\end{abstract}

Charlotte Rahn collected data in Uganda. 


\subsection{INTRODUCTION}

In primates, as well as in anurans, birds and some other mammals, the environment is known to be a potential factor of variations in vocal behaviour (Chapter 2). Animals might adjust usage and/ or acoustic structure of their vocalisations to increase the probability of detection by the receiver and optimise propagation. Such strategies should occur most often in vocalisations used for long-distance communication. These are intended to travel over large distances while being in contact with the environment (Brown et al. 1995; Mitani \& Stuht 1998) and they cannot be relayed by the visual channel (Marler 1974, 1977; Waser 1982; Brown et al. 1995), in contrast to vocalisations used for short-distance communication (see Chapter 4).

While the acoustic structure of vocal signals is considered to be largely innate, their usage is known to be more flexible in primates (see General introduction). The influence of the environment is therefore expected to be larger in the later than in the former. This is the case in four primate species on Siberut Island, Indonesia. The acoustic structure of loud calls of Kloss gibbons, Mentawai macaques, Mentawai leaf monkeys and pig-tailed langurs appears to be genetically fixed, whereas the time of calling is adjusted to ambient noise level (Schneider et al. 2008).

In the previous chapter (Chapter 4), environment-related variations in vocal behaviour were examined in contact calls used over short distances (i.e., grunts) in olive baboons. The next logical step is then to study this question in vocalisations used for long-distance communication, that is, clear barks and loud calls. Unfortunately, the baboons under study in Nigeria and in Uganda (same troops as in Chapter 4) uttered these calls rarely (Chapter 1). The limited set of audio-recordings of sufficient quality does not permit any investigations of environmental influences on acoustic structure. The present study is therefore restricted to usage. It investigates whether olive baboons show some plasticity in usage of clear barks and loud calls to respond to variations in structural and acoustic features of the habitat in which they vocalise. Vocal behaviour is considered at the group level, and not at the individual level like in the previous chapter, because of the low emission rate for each call type. In addition, data concerning loud calls were collected only in Nigeria since Ugandan baboons were not heard to utter them.

Call rates for clear barks and occurrences and timing of loud calls were considered. The timing of clear barks was not investigated because these calls are bound to a very specific context (i.e., separation from the rest of the troop or from particular individuals) which is 
unlikely to be correlated with time of day. In contrast, loud calls are uttered in a wider range of situations, including spontaneously (Chapter 1). Loud call emissions might therefore be less dependent on the occurrence of a particular context and therefore more likely to be flexible in their time of occurrence than clear barks. The distribution of the proportion of travel between habitat types was also examined because it might influence clear bark usage (Rendall et al. 2000). Clear barks and bouts of loud calls were expected to be given more often in a closed environment such as forest where visibility is reduced and sound attenuation more important (Chapter 3) than in an open environment such as woodland savannah or grassland. Giving calls more frequently should increase the likelihood of attenuated calls to be detected by potential recipients (e.g., Waser \& Waser 1977). Loud calls should be uttered preferentially when background noise is the lowest, that is, the most favourable for sound transmission and detection (e.g., Waser \& Waser 1977; Henwood \& Fabrick 1979). Animals were then expected to call more often at the beginning of the morning than at the end, since background noise level tends to increase over the morning (Chapter 3).

\subsection{MATERIAL AND METHODS}

\subsubsection{Study sites and animals}

Data collected in the two previously described troops of Nigerian olive baboons Gamgam and Kwano troops- ranging in Gashaka-Gumti National Park, Nigeria (chapters 1 and 4) were combined with those collected in Sonso troop in Budongo Forest Reserve, Uganda (see Chapter 4). As in the previous chapter, two types of habitat were distinguished: forest and open habitat (data collected at the border between them or in other vegetation formation were not considered). The closed habitat type -hereafter forest- presented a low visibility and a high attenuation rate of sounds of high frequencies. It encompassed all forested areas. The open habitat type included woodland savannah in Nigeria and grassland in Uganda. The visibility was higher and attenuation of high frequencies lower in this habitat type than in forest on average (Chapter 3; see also Appendix 4). Variations in grass height within woodland savannah were not considered in the present chapter because baboons often utter clear barks and loud calls from high perches (i.e., above grass level; Chapter 1). Both habitat types tended to have a lower ambient noise level early in the morning than at the end of the morning in Nigeria (Chapter 3). 


\subsubsection{Data collection}

I collected data for this study over 8.5 months distributed over two dry seasons (Feb May 06 and Nov 06 - Apr 07) in Gamgam troop (240 h of observation) and Kwano troop (322.5 h of observation) in Nigeria. Data presented for loud calls were only collected in Nigeria in Dec 06 - Apr 07. C. Rahn collected data in Sonso troop in Uganda over 2.5 months in Apr - Jul 07 (173.5 h of observation). Clear barks and loud calls were recorded ad libitum from all baboons of the three troops in forest and open habitat during daily focal follows. All occurrences of calls were noted in forest and in open habitat (clear barks: 207 in Gamgam troop, 745 in Kwano troop and 283 in Sonso troop; loud call bouts: 17 in Gamgam troop and 20 in Kwano troop). Focal follows lasted from 6:00 to 12:00 in Nigeria and 15 min distributed between 8:00 and 17:00 in Uganda. Focal observations rotated between 1 adult male and 5 adult females in Gamgam troop, 3 adult males and 10 adult females in Kwano troop and 3 males ( 1 adult and 2 subadults) and 7 adult females in Sonso troop. Scans for behavioural data were done every $15 \mathrm{~min}$ in Nigeria and every $3 \mathrm{~min}$ in Uganda. For each sampling point, habitat type and activity of the focal animal (see Chapter 1 for definitions) were recorded. In the present chapter, only the distribution of the activity "travel" (i.e., walking, running, climbing in trees) between the two habitat types from all focal animals and the distribution of time spent by adult males in forest and open habitat were considered since they might be related to usage of clear barks and loud calls.

As clear barks were uttered rarely (in comparison to grunts; see Chapter 1), a "group" bark rate was calculated by counting all clear barks within earshot during focal follows in forest and open habitat and relating this number to the time spent in each habitat type. This represents an estimation of how many calls a baboon might hear, since the main energy of barks $(0.3-10 \mathrm{kHz})$ falls within the hearing range of baboons $(0.04-40 \mathrm{kHz})$ and humans (0.03 - 17.6 kHz; Heffner 1998; Heffner 2004). To test the influence of habitat type, bark rates were calculated on days for which data in both forest and open habitat were available (Gamgam: 42 days; Kwano: 60 days; Sonso: 19 days). They were corrected for troop size. Loud calls were given even more rarely than clear barks (Chapter 1). No reliable call rate could be calculated for these calls in each habitat type, and therefore only occurrences of bouts were considered. 


\subsubsection{Statistical analyses}

The distribution of the activity "travel" for each focal animal was compared between forest and open habitat in each troop separately by using exact 2-tailed Wilcoxon T-tests. Bark rates were compared between the three troops by using exact 2-tailed Mann-Whitney Utests. To examine the influence of the habitat on bark rate, a general linear model (GLM) with repeated measures (repetitions over days) was used in each troop separately, with habitat type as a within-subject factor. A Chi-square test was used in each troop separately to compare the number of observed occurrences of loud call bouts with the number of expected occurrences according to the time spent in each habitat type by adult males. This time was estimated by the proportion of scans in each habitat type. Statistical analyses were done with the software SPSS 15.0 for Windows, and the significance level was set at 0.05 . The distribution of loud call bouts over the day was only described.

\subsection{RESULTS}

\subsubsection{Behavioural data}

Gamgam baboons travelled on average ( \pm SD) in $9.9 \pm 5.2 \%$ of scans in forest and in $20.8 \pm 4.0 \%$ of scans in open habitat but the difference was not significant (exact Wilcoxon T-test: $\mathrm{N}=6, \mathrm{~T}=1, \mathrm{p}=0.063)$. Kwano baboons travelled on average $( \pm \mathrm{SD})$ in $14.0 \pm 5.3 \%$ of scans in forest and in $18.5 \pm 10.8 \%$ of scans in open habitat, but the difference did also not reach significance (exact Wilcoxon T-test: $\mathrm{N}=13, \mathrm{~T}=22, \mathrm{p}=0.110$ ). In contrast, baboons of Sonso troop travelled significantly more in forest (mean \pm SD: $17.5 \pm 3.7 \%$ of scans in forest) than in open habitat (mean \pm SD: $10.8 \pm 4.9 \%$ of scans in open habitat; exact Wilcoxon Ttest: $\mathrm{N}=10, \mathrm{~T}=0, \mathrm{p}=0.002)$.

\subsection{2 $\underline{\text { Clear barks }}$}

Since bark rates differed significantly between the three troops (exact Mann-Whitney U-tests: Gamgam vs. Kwano: $\mathrm{N}_{1}=42, \mathrm{~N}_{2}=60, \mathrm{U}=734.5, \mathrm{p}<0.001$ [see also Chapter 1]; Gamgam vs. Sonso: $\mathrm{N}_{1}=42, \mathrm{~N}_{2}=19, \mathrm{U}=103.5, \mathrm{p}<0.001$; Kwano vs. Sonso: $\mathrm{N}_{1}=60$, $\mathrm{N}_{2}=19, \mathrm{U}=264.5, \mathrm{p}<0.001$ ), analyses concerning the influence of the habitat were run separately in each troop. Bark rates did not vary significantly according to the habitat type in any of the three troops (GLM with repeated measures: Gamgam: $\mathrm{N}=42, \mathrm{~F}=0.051$, 
$\mathrm{p}=0.823 ;$ Kwano: $\mathrm{N}=60, \mathrm{~F}=1.769, \mathrm{p}=0.189 ;$ Sonso: $\mathrm{N}=19, \mathrm{~F}=2.373, \mathrm{p}=0.141$ Figure 5.1).

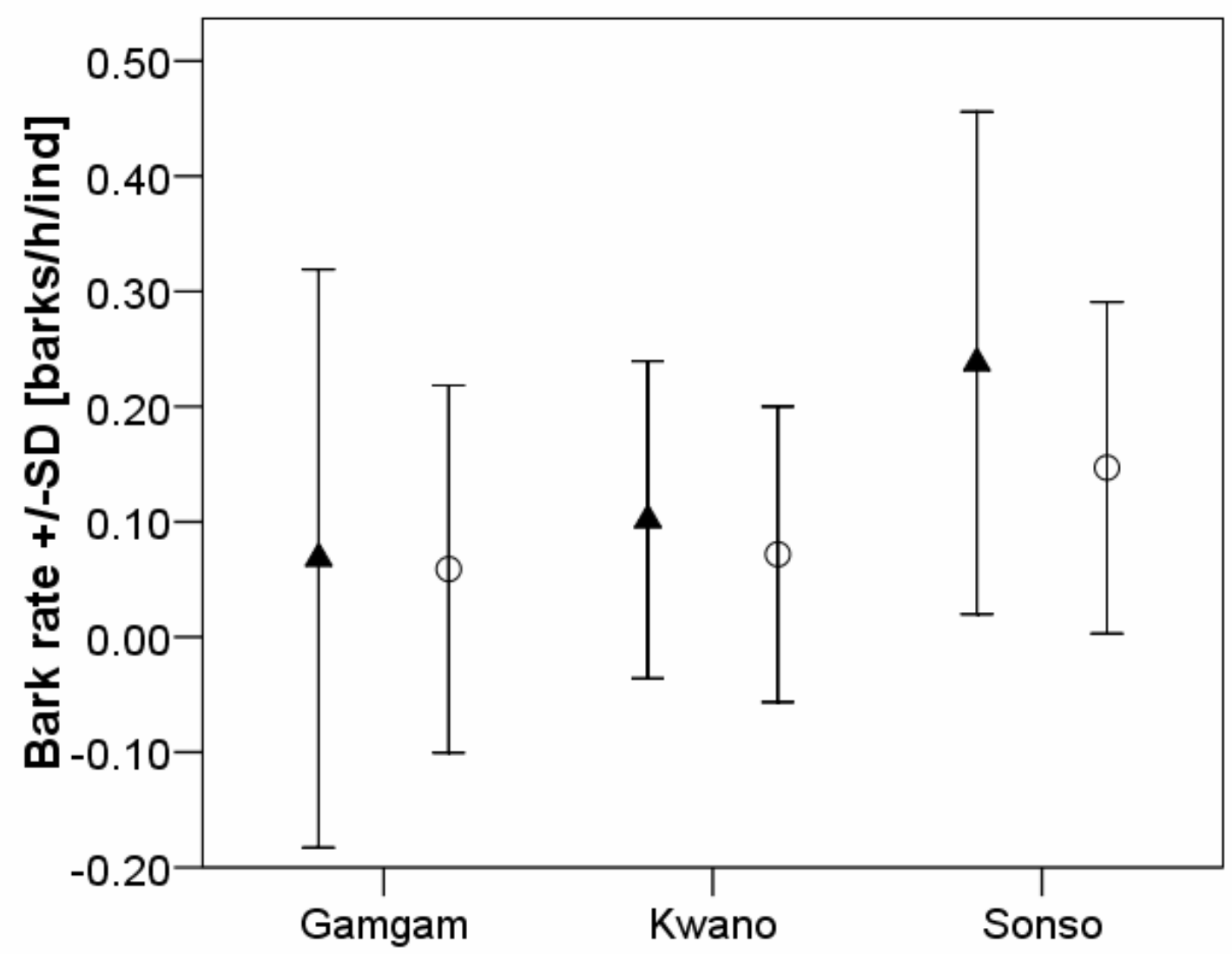

Figure 5.1: Mean (over days) and standard deviation of bark rate (corrected for troop size) in forest (black triangles) and open habitat (circles) in Gamgam, Kwano and Sonso troops.

\subsubsection{Loud calls}

Most bouts were uttered in woodland savannah in Gamgam troop and in forest in Kwano troop. The observed distribution of bouts did not differ from the expected distribution according to the time spent by adult males in forest and woodland savannah (Chi-square test: Gamgam: $\mathrm{X}^{2}=0.011, \mathrm{p}=0.916$; Kwano: $\mathrm{X}^{2}=0, \mathrm{p}=1$; Table 5.1). 
Table 5.1: Proportion (\%) of time spent by adult males in forest and woodland savannah, and number (\#) of loud call bouts expected according to the time spent in each habitat type and of bouts actually observed in each habitat type in Gamgam and Kwano troops.

\begin{tabular}{l|l|cc}
\multicolumn{2}{c|}{} & Forest & $\begin{array}{c}\text { Woodland } \\
\text { savannah }\end{array}$ \\
\hline \multirow{3}{*}{ Gamgam } & \% time & 30.6 & 69.4 \\
& \# bouts expected & 5.2 & 11.8 \\
& \# bouts observed & 5 & 12 \\
\hline \multirow{3}{*}{ Kwano } & \% time & 75.2 & 24.8 \\
& \# bouts expected & 15.0 & 5.0 \\
& \# bouts observed & 15 & 5
\end{tabular}

Most loud call bouts were given before 10:00 in the morning (in hour 6, 7, 8 or 9; Figure 5.2).

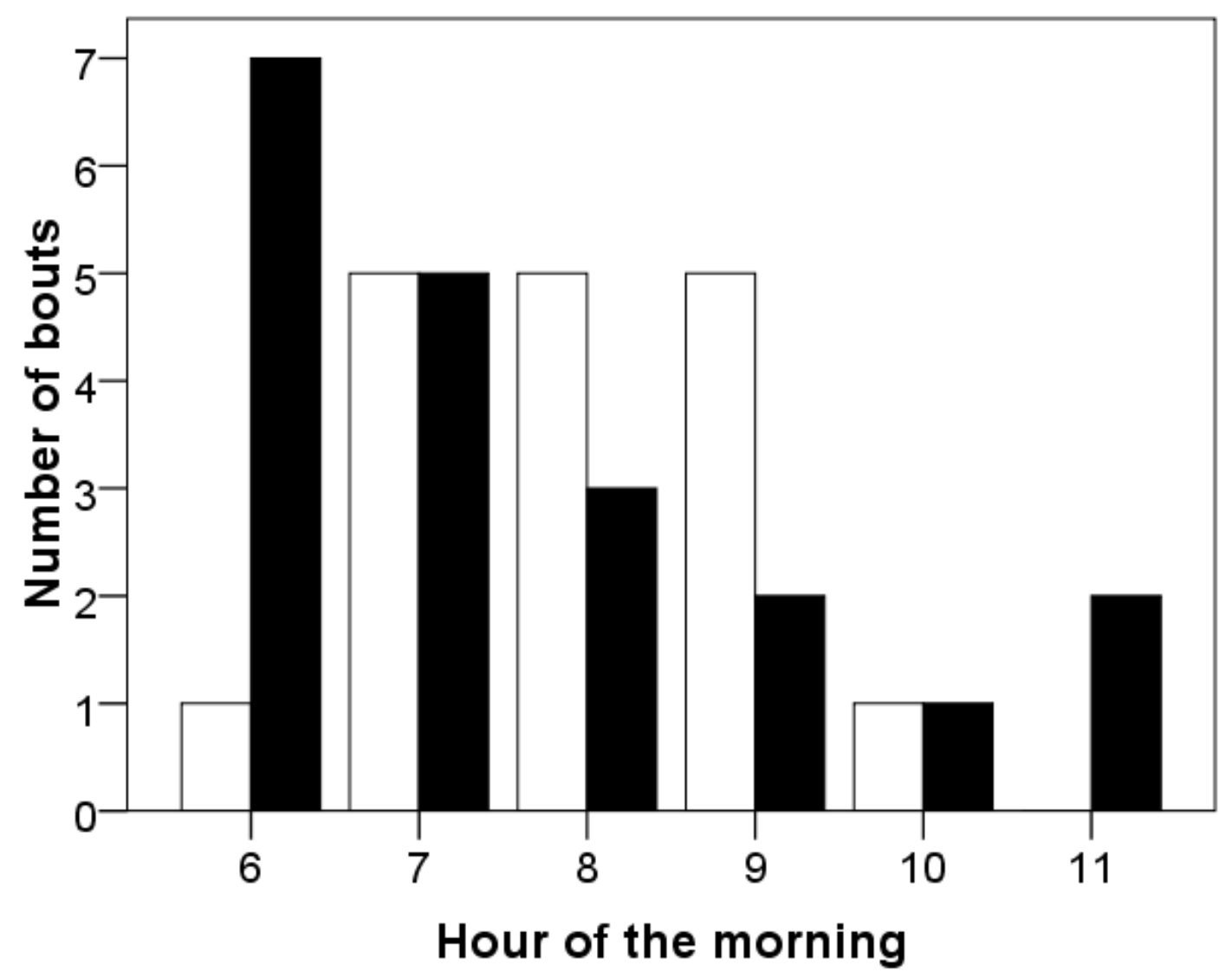

Figure 5.2: Distribution of loud call bouts over the morning hours (ex: hour $6=$ from 6:00 to 6:59) in Gamgam (white bars) and Kwano (black bars) troops. 


\subsection{DISCUSSION}

This chapter examined whether olive baboons of three troops adjusted usage of clear barks and loud calls to environmental conditions. Bark rate did not vary significantly according to habitat type and therefore to visibility in any of the three troops. Concerning loud calls, the distribution of these calls in forest and woodland savannah was reversed between the two Nigerian troops and corresponded to the proportion of time spent by males in each habitat type. Nevertheless, adult males tended to utter most of these calls in the first half of the morning, when ambient noise was slightly quieter.

Group bark rate (corrected for troop size) did not vary significantly according to the habitat type in which baboons of the three troops ranged. These baboons do not seem to adjust their call rate to visibility conditions in their environment. Results should nevertheless be confirmed by examining individual bark rates, which should allow more precise analyses with control on the caller's identity. Collecting reliable data will take a long time, given the very low bark rates in these troops, but this is nevertheless worth since it would increase the power of the investigation. To explain the absence of variations with habitat type in bark rate, the hypothesis might come that clear barks are so loud that repeating them is not necessary because they would be heard anyway from any part of the home range. The balanced bark rate between habitat types might also be explained by the fact that animals do usually not receive much feedback from their calls since clear barks are seldom answered (Cheney et al. 1996). Therefore, individuals cannot experiment the benefit (being answered more quickly) of calling more often in an environment where sound propagation is more difficult. However, these two hypotheses do not hold in another baboon taxon. Contrary to olive baboons in Nigeria and Uganda, chacma baboons in Botswana utter clear barks at a higher rate in a habitat where visibility is reduced than in a more open environment (Rendall et al. 2000). This finding suggests that bark rate might be regulated indirectly by environmental factors (see hereafter).

Bark rate of chacma baboons was calculated for each individual (Rendall et al. 2000), whereas those of Nigerian and Ugandan olive baboons were calculated for the whole group and only afterwards corrected for troop size. The way of calculating call rate (individual vs. group) cannot account for the contrasting results between baboon taxa since comparisons between habitat types were made within troops. One potential explanation is that the populations differ in their general usage of clear barks. Even if the contexts of utterances were 
found to be similar between the two taxa (Chapter 1), differences might lie in the frequency at which calls are uttered in the particular context (separation from conspecifics) which usually elicits them. Olive baboons in Nigeria (pers. obs.) and in Uganda (Rahn 2008) do not have serious predators anymore (except humans to some extent). They might therefore remain relatively relaxed when ranging on their own (i.e., separated from the rest of the group) for a while in either habitat type. This would explain the low rate of barking in these two populations (see Chapter 1) and the absence of variations in bark rate according to the habitat type. In contrast, chacma baboons studied in Botswana undergo a strong predation risk (Cheney et al. 2004), which might be even higher in an environment with low visibility offering many hiding places for predators. These baboons might then feel highly aroused when separated from the rest of the group and might therefore utter clear barks at an especially high rate in a habitat with low visibility where risk is highest. The influence of the habitat type on bark rate would therefore be indirect through predation risk and arousal level. Future studies should estimate bark rates in olive baboon populations which undergo a high predation pressure and in chacma baboon populations which have a low predation risk to test this explanation. If possible, bark rates in these populations should also be compared between habitats with various visibility conditions.

Other factors are implicated in bark rate regulation and they might also constrain flexibility as a response to the environment. The influence of those related to the status of the caller (presence and age of offspring, social rank; Cheney et al. 1996; Rendall et al. 2000) cannot be considered further since bark rate was investigated at a group level in the present chapter. Other factors related to the situation of the caller in its environment (i.e., location within the home range, proximity to other group members, and travel rate; Cheney et al. 1996; Rendall et al. 2000) are discussed hereafter (one should nevertheless note that behavioural data were collected from focal animals and bark rate from the whole group in this chapter, which might lead to some shifts between the two data sets). Firstly, a higher call rate is expected when individuals are near the periphery of their home range than when they are in its core (Rendall et al. 2000). Unfortunately, the influence of the location within the home range cannot be tested in the present chapter because the present data did not include any information on spatial distribution of callers within the home range. Secondly, a low bark rate is expected when callers are in proximity to other group members (Rendall et al. 2000). According to these results in chacma baboons, olive baboons are expected to call more often when they are more scattered, that is, more likely to get separated from the rest of the group. However, when comparing bark rates between habitat types, one should consider that 
visibility also varies. Indeed, because of the high vegetation density in forest, an individual might lose visual or auditory contact with its conspecifics more rapidly in forest than in open habitat (see also Koda et al. 2008). The likelihood of losing contact with the rest of the group might therefore explain bark rate more reliably than group spread. For instance, in the present chapter, group spread itself did not follow the absence of variations in bark rate. In Kwano troop, group spread did not vary significantly between habitat types (Chapter 1), as did bark rate. However, Gamgam baboons were more scattered in woodland savannah than in forest (Chapter 1), while bark rate did not vary significantly between habitat types (no comparable data were available from Sonso troop). Group spread is therefore an unlikely explicative factor of bark rate in these troops. However, it was only measured within $10 \mathrm{~m}$ of the focal animal. Extending this range up to $20 \mathrm{~m}$ in future studies might be meaningful considering the context in which clear barks are uttered. Finally, another factor is travel rate. The high bark rate of chacma baboons in areas with low visibility can be explained by a higher rate of travel in this environment, in comparison to a more open habitat type. The propensity of travel leads to an increased likelihood of losing contact with the rest of the group and therefore of uttering clear barks (Rendall et al. 2000). In the present study, the distribution of the activity "travel" is balanced between habitat types in Gamgam and Kwano troops. This could explain the absence of variations with habitat type in bark rate, since olive baboons are not more likely to get separated from the troop in forest than in woodland savannah. In addition, olive baboons from Uganda, which show a non-significant tendency to bark more often in forest than in grassland, travel significantly more in forest. This last pattern would therefore resemble the one in chacma baboons. At first sight, travel distribution might then be a potential predictor of bark rate, like travel rate in chacma baboons. However, one restriction comes from a closer examination of data from Nigeria. In both troops, baboons have a non-significant tendency to travel more in woodland savannah than in forest, whereas bark rates tended (nonsignificantly) to be higher in forest than in woodland savannah (Figure 5.1). This trend is then contrary to what would predict the preceding argumentation. This last remark suggests that the relationship between travel and bark rate is complex. Future studies should investigate this relationship more precisely on individual bark rates in olive baboons, in order to be able to relate precisely travel activity and call rate within individuals.

The Nigerian baboons under study seem to avoid high ambient noise level at the end of the morning to utter loud calls. Such a tendency was also found in Siberut Island, Indonesia, where Kloss gibbons, Mentawai macaques, Mentawai leaf monkeys and pig-tailed langurs utter loud calls mostly early morning when ambient noise is low (Schneider et al. 
2008). In addition, in the present chapter, habitat type does not seem to have an influence on usage of loud calls. The distribution of occurrences of loud call bouts between forest and woodland savannah reflects the usage of habitat type. Gamgam males, which spent most of their time in woodland savannah, uttered more loud calls in this habitat type, whereas Kwano males, which ranged mostly in forest, give more loud calls there. These results suggest that male baboons do not have a preferred habitat type to utter loud calls. They nevertheless still take advantage of the most favourable acoustic conditions in the timing of their calls, as far as the context of calling allows some flexibility. However, these conclusions are based on a limited set of data. Further similar investigations (for both time and place of loud call utterances) are required to strengthen them. Most importantly, the rate at which loud call bouts are uttered should be considered, since this would allow to control for the amount of time spent in each habitat type.

The present results suggest that usage of clear barks is constrained by the context of emission (separation from the group or from particular individuals in a more or less risky environment) and therefore is only indirectly and minimally affected by habitat type. In contrast, some flexibility occurs in utterances of loud calls and allows baboons to avoid unfavourable ambient noise conditions. Future studies should examine whether baboons compensate these limited adjustments in usage by adjusting the acoustic structure of their vocalisations, in order to optimise sound propagation. Clear barks and loud calls carry identity cues of the caller (Fischer et al. 2001a, 2002; Kitchen et al. 2005). These identity cues are salient since baboons respond more often to clear barks of related individuals than to those of non-relatives in the rare answers that they give when they are themselves at risk of being separated from the group (Cheney et al. 1996) and adult males react differently according to the callers implicated in a contest bout (Kitchen et al. 2005). It might then be profitable for a caller to provide identity cues to a potential recipient to get an answer from a relative in the case of clear barks or to discourage other males to approach in the case of loud calls. However, call propagation through the environment might lead to degradation of some acoustic features and, among others, those related to individuality (but not necessarily; see Lameira \& Wich 2008). There might therefore be an advantage for the caller to adjust the structure of its calls in order to minimise their degradation. Unfortunately, clear barks and loud calls were uttered rarely in the three troops under study (Chapter 1). Too few clear barks and loud calls could be recorded from different individuals in the two habitat types to analyse variations in their acoustic structure. Further investigations should concentrate efforts on collecting audio-recordings of these vocalisations from different individuals in various habitat 
types. Acoustic features of these calls could then be compared within individuals between habitat types while controlling caller identity, as for grunts in Chapter 4.

In summary, baboons show a limited degree of flexibility by adjusting the time of emission of loud calls to ambient noise level, while the context in which clear barks are uttered seems to override influences of environmental conditions. Future investigations should consider individual call rates and examine whether baboons compensate limited adjustments in usage by adapting the acoustic structure of these vocalisations to minimise environmental influences during their propagation. 


\section{General conclusion}

This thesis focuses on the vocal behaviour of two troops of olive baboons in Nigeria. Comparing usage and structure of contact calls between olive baboon populations and between baboon taxa revealed more variability in call usage than in call structure, as predicted. A review of studies in anurans, birds and mammals showed that environmentrelated adjustments in vocal behaviour between species or populations are generally in line with predictions based on sound propagation mechanisms but not as widespread as expected across taxa. This question was examined at an intra-individual level in olive baboon vocal behaviour. Individuals of the two Nigerian troops and of a Ugandan troop showed some flexibility according to visibility conditions in usage of contact calls and more surprisingly in structure of vocalisations used in communication over short distances. However, other factors such as group spread and context of calling seemed to constrain this environment-related plasticity. All these results have been discussed in the corresponding chapters of this thesis. Therefore, in this short general conclusion, I only briefly comment the two major findings about intra-individual flexibility in grunt structure and variability between baboon populations and taxa in usage and structure of contact calls. Then, I suggest directions for future investigations. Finally, I present some implications of the present thesis concerning knowledge about the evolution of speech.

\section{Lengthening of grunts in forest}

In the present thesis, variation in grunt duration according to habitat visibility is the most robust finding since it occurs in two populations of olive baboons (Chapter 4). This flexibility is in accordance with predictions for optimised transmission and better detection by a receiver but it is still surprising. Such adjustments occurring in both populations were expected in usage of vocalisations, especially of those used for long-distance communication, but it was not the case. Adjustment in call rates did not occur for clear barks and loud calls (Chapter 5) and were not systematic for grunts (Chapter 4). In contrast, baboons of both populations were surprisingly adaptive and took not only some features of their immediate environment but also the contexts in which they grunted into account to adjust the duration of their signals accordingly (Chapter 4). This shows that some flexibility is possible within the 
phylogenetically determined acoustic structure of species-specific vocalisations. Vocal production is therefore mostly but not completely fixed.

Olive baboons seem then to control the rate of emission of their grunts to a certain extent (according to results from Uganda). Most importantly, they have some control on their vocal production organs, and especially on those implicated in the regulation of duration. A neural basis for this control on vocal usage and production might be the cingulated pathway (Sutton et al. 1974; Jürgens 2002). This phylogenetically old neural system comprising limbic structures connected to the peri-aqueductal grey in mid-brain is shared between primates and humans. It allows some limited control on production of species-specific vocalisations (limited range of variability in structure of species-specific vocalisations and decision about uttering different variants or not; Jürgens 2002). A phylogenetically recent system, the neocortical pathway, has a restricted development in primates and is most developed in humans. It includes the cerebellum, part of the thalamus, basal ganglia and the motor cortex. This recent system is indispensable for voluntary control on manual gestures, but its development for voluntary control on the phonatory apparatus occurs only in humans (Jürgens 2002; Ploog 2002). In primates, it does not seem to be implicated in control over learned vocal patterns (Sutton et al. 1974; Ghazanfar \& Rendall 2008) or in production of genetically determined vocalisations (Jürgens 2002; see introduction in Hage 2005; Ghazanfar \& Rendall 2008). Features of these two neural systems suggest that baboons use only the limited range of variability allowed by the limbic system (cingulated pathway) to adjust the duration of their grunts since the general acoustic structure of grunts is phylogenetically determined (see Chapter 1 , in which only minor variations probably related to body constitution were found in grunt structure between baboon taxa). This neural pathway seems to permit some plasticity mostly in temporal parameters of primate vocalisations, as only Sutton and colleagues (1973) reported a successful conditioning of coo call duration in rhesus macaques. No other study investigating conditionability of other acoustic variables has been found.

Control on respiratory components of phonation might stem mainly from pre-motor neurons from the nucleus retroambiguus, but also from other premotor areas connected collectively with all phonatory motoneurone pools (e.g., ventrolateral parabrachial region, lateral pontine reticular formation, parvocellular and gigantocellular nuclei of the rostra medulla and the dorsal and ventral reticular nuclei of the caudal medulla; Jürgens 2002). Control on respiratory organs implicated in phonation stems then from multiple neural structures. This control might be phylogenetically older than the one on phonatory (mainly regulating frequency characteristics) or filter (mainly regulating energy distribution 
characteristics) systems (Janik \& Slater 1997, 2000). This could explain why duration is an acoustic variable preferentially showing intra-individual short-term variation in grunts (Chapter 4), in comparison to fundamental frequency or peak frequency which appear to be under fine voluntary control in humans only (Jürgens 2002). The precise degree of control that monkeys have on their vocal production remains nevertheless to be further investigated. Neural processes implicated in the intra-individual flexibility highlighted in this thesis might be investigated by trying to condition other acoustic variables than duration such as fundamental frequency or peak frequency, as well as by studying the ontogeny of the flexibility found in baboons, as already suggested in Chapter 4.

\section{$\underline{\text { Variability between baboon populations and taxa }}$}

The present thesis broadens knowledge on baboon vocal communication by characterising the vocal behaviour of olive baboons and comparing findings with those in other olive baboon populations and in other baboon taxa (mostly chacma baboons). Contact call structures present only minor variations between populations or taxa, as expected (Chapter 1). Such findings corroborate the assumption of a genetic constrain on vocal production, which defines an acoustic frame for species-specific calls (see General introduction). A larger inter-population variability was found in usage of contact calls, especially in call rate (Chapter 1), but also in its flexibility in response to environmental conditions (Chapter 4). This variability is supposedly related to the interplay of several factors such as contexts of calling, group spread, social structure and predation risk in relation to environmental conditions (see discussions in chapters 1, 4 and 5). Baboons of different populations and taxa are then flexible in their usage of contact calls, as expected, and seem to respond differentially to various ecological and social constrains. This opportunism and adaptability might explain baboons' success over such a large range in sub-Saharan Africa (Henzi \& Barrett 2003).

\section{$\underline{\text { Suggestions for an integrative study }}$}

This thesis suggests that social and ecological factors interplay in baboon vocal behaviour. Several directions for future investigations have already been evoked, and they are here summarised for the long-term project to which this thesis contributes. It aims to integrate 
various factors (phylogenetic relationships, social system, and environmental conditions) to understand their interplay in baboon communication system.

Combining studies in different populations should help to understand the interplay of the above-cited factors. Other troops in which data will be collected should therefore be carefully selected. One important criterion is phylogenetic relatedness. Recent studies (e.g., Zinner et al. 2008) shed some additional light on phylogenetic relationships between baboon taxa and populations. Using this information allows to control for genetic influences on vocal behaviour and to avoid missing interesting genetically different (but otherwise quite similar) populations. The present thesis used this information to compare olive baboons from two populations showing the same morphotype and social system and ranging in similar habitats but differing in their mitochondrial DNA as much as two subspecies. In addition, one should select study groups in a way providing various combinations of social systems and environmental conditions. For instance, comparing the vocal behaviour (especially call rates) of baboon troops living in similar environmental conditions (with similar visibility), but showing different degree of fission-fusion would allow to check whether a high frequency of fission-fusion implies a high call rate. It could also give some cues on the extent at which habitat visibility interplays with this factor.

It is essential for such an integrative project that methods used to collect data be comparable between study groups. These methods should be inspired from the present thesis and complemented. Other factors (e.g., proximity to group members, general group spread, tendency for fission-fusion) than the ones actually recorded appeared to potentially play a role in baboon vocal behaviour. Future studies should therefore additionally evaluate the effects of these factors. Data about emission rate and audio-recordings suitable for acoustic analyses should be collected. For each recorded vocalisation, at least actual (sub)group size, habitat type, time, caller identity, proximity to other group members, and context of calling should be noted. In the same time, behavioural data from focal sampling (habitat type, activity of the focal animal, group spread) should also be collected. Special attention should be paid that all data fit together, that is, that behavioural data can be coupled with audio-recordings. These data should also be complemented by general information about the social system of the study group, its size, its composition, the rate of social interactions and predation risk.

If such a large data set about baboon vocal behaviour could be built, a study integrating various potentially influential factors (phylogenetic relatedness, social system, environmental conditions) should unravel their interplay in the regulation of usage and in the determination of acoustic structure of contact calls in baboons. Comparisons should be 
conducted at different levels, as it was done in this thesis. Comparisons of populations from different taxa highlight phylogenetic influences, but still allow to test the effects of social or environmental factors, as well as their potentially differential degree of effectiveness in various taxa. Comparisons might also concern different populations within a taxon. Increasing the number of populations studied within each taxon improves control on various factors and allows to refine analyses on precise aspects of genetic, social or environmental influences. Finally, as in the present thesis, available data might also be used to test the influence of the social surrounding or environmental conditions within troops. This would further document the degree of intra-individual flexibility in baboon vocal behaviour. A long-term project with these various levels of comparisons will probably not permit unravelling all potential influences on vocal behaviour, but it will still provide useful insights on evolutionary constrains in the shaping of vocal communication systems.

\section{Implications for knowledge on the evolution of speech}

Human vocal communication system shares many features such as mechanisms of sound production, basic neural structures but also to some extent referential ability with some primate communication systems (Fitch 2000; Lieberman 2002). A large number of publications approached the issue of the evolution of speech, and many highlighted the legitimacy of a comparative approach (e.g., Wind et al. 1992; Hauser 1996; Ghazanfar \& Hauser 1999; Fitch 2000, 2005; Ghazanfar \& Rendall 2008). Studying vocal communication in primates supplements data from fossil records and from investigations of the neuroanatomy of related species (de Boer 2005). However, comparative studies could shed light on the emergence of only some characteristics of speech. Neuro-anatomical investigations suggest that primate vocalisations can be used as reliable models for non-verbal emotional vocal utterances in humans but not directly for speech (Jürgens 2002).

Vocal plasticity is a prominent feature of speech. The intra-individual flexibility in response to variations in environmental conditions found in this thesis in duration of olive baboon grunts parallels some features of human whistled languages. These forms of speech can be described as a transposition of a spoken language into a repertoire of whistles. They are used mainly in forests, mountains and valleys to communicate outdoor over large distances, much larger than those reached with normal speech (Meyer 2004, 2005). In such environments in which sound transmission is difficult and reverberation substantial (like in forest in the present thesis), the whistler tends to increase the length of its sentences, words 
and even vowels when he aims to communicate over greater distances. Variations in duration might be the most efficient way of increasing propagation distance since frequency and pitch modulations through the shaping of the vocal tract (or of the used tools such as fingers or leaves) are mainly used for element recognition in these languages. Lengthening vowels is presumed to increase intelligibility (Meyer 2005), as lengthening grunts is supposed to increase propagation distance and localisability (Chapter 4).

With this parallel, the present thesis confirms that intra-individual plasticity is not specific to speech, even if it is more restricted in primates. This limitation lies in differences in neural mechanisms. Primates lack elaborated voluntary control through the motor cortex on their vocal apparatus (Jürgens 2002). They also do not show strong connections with motoneurones innervating vocal production organs from basal ganglia, which are responsible for the ability of responding to new circumstances to allow a change in direction of thoughts or motor response as opposed to routine circumstances (Lieberman 2002). Nevertheless, the similarity in primates and humans in the ability to adjust call duration to respond to variations in environmental conditions suggests that their common ancestor already possessed some degree of flexibility in vocal production. This is a pre-requisite for vocal learning and for vocal imitation, the last one clearly distinguishing speech from primate vocal communication (Fitch 2000). This flexibility in vocal production might represent a precursor of the important plasticity in speech. However, the plasticity in duration highlighted here is still far away from the very precise breath control required for speech (MacLarnon \& Hewitt 1999; Ghazanfar \& Rendall 2008). The hypothesis that some neural bases necessary to regulate speech evolved gradually, before the appearance of anatomical structures of modern human beings allowing the current complexity in speech production (e.g., descended larynx, highly mobile tongue; Lieberman 2002; Ghazanfar 2008) is then supported by the evidence of intra-individual flexibility in vocal production brought by the present thesis. 


\section{References}

Akinsoji A. 1996. Vegetation Types and Ethnobotanical Studies of Gashaka Gumti National Park, Nigeria. University of Lagos. Report for WWF-UK, NCF.

Altmann J. 1980. Baboon Mothers and Infants. Harvard University Press, London.

Altmann S. A., Altmann J. 1970. Baboon Ecology - African Field Research. Karger, Basel.

Anderson M. E., Conner R. N. 1985. Northern cardinal song in three forest habitats in Eastern Texas. The Wilson Bulletin 97: 436-449.

Aylor D. 1971. Noise reduction by vegetation and ground. Journal of the Acoustical Society of America 51: 197-205.

Aylor D. 1972. Sound transmission through vegetation in relation to leaf area density, leaf width, and breadth of canopy. Journal of the Acoustical Society of America 51: 411414.

Badyaev A. V., Leaf E. S. 1997. Habitat associations of song characteristics in Phylloscopus and Hippolais warblers. The Auk 114: 40-46.

Baker M. C. 2006. Differentiation of mating vocalizations in birds: acoustic features in mainland and island populations and evidence for habitat-dependent selection on songs. Ethology 112: 757-771.

Bergman T. J., Beehner J. C. 2004. Social system of a hybrid baboon group (Papio anubis $\times$ P. hamadryas). International Journal of Primatology 25: 1313-1330.

Bergmann H.-H. 1978. Beziehungen zwischen Habitatstruktur und Motivgesang bei europäischen Grasmücken (Gattung Sylvia). Journal für Ornithologie 119: 236-237.

Bertelli S., Tubaro P. L. 2002. Body mass and habitat correlates of song structure in a primitive group of birds. Biological Journal of the Linnean Society 77: 423-430.

Blumenrath S. H., Dabelsteen T. 2004. Degradation of great tit (Parus major) song before and after foliation: implications for vocal communication in a deciduous forest. Behaviour 141: 935-958.

Blumstein D. T., Turner A. C. 2005. Can the acoustic adaptation hypothesis predict the structure of Australian birdsong? Acta Ethologica 8: 35-44.

Boinski S. 1991. The coordination of spatial position: a field study of the vocal behaviour of adult female squirrel monkeys. Animal Behaviour 41: 89-102.

Boncoraglio G., Saino N. 2007. Habitat structure and the evolution of bird song: a metaanalysis of the evidence for the acoustic adaptation hypothesis. Functional Ecology 21: 134-142.

Bosch J., de la Riva I. 2004. Are frog calls modulated by the environment? An analysis with anuran species from Bolivia. Canadian Journal of Zoology 82: 880-888.

Bowman R. I. 1979. Adaptive morphology of song dialects in Darwin's finches. Journal für Ornithologie 120: 353-389.

Bradbury J. W., Vehrencamp S. L. 1998. Principles of Animal Communication. Sinauer associates, Sunderland, Massachusetts. 
Braune P., Schmidt S., Zimmermann E. 2005. Spacing and group coordination in a nocturnal primate, the golden brown mouse lemur (Microcebus ravelobensis): the role of olfactory and acoustic signals. Behavioural Ecology and Sociobiology 58: 587-596.

Brenowitz E. A. 1986. Environmental influences on acoustic and electric animal communication. Brain Behaviour and Evolution 28: 32-42.

Brown C. H., Maloney C. G. 1986. Temporal integration in two species of Old World monkeys: blue monkeys (Cercopithecus mitis) and grey-cheeked mangabeys (Cercocebus albigena). Journal of the Acoustical Society of America 79: 1058-1064.

Brown C. H., Waser P. M. 1988. Environmental influences on the structure of primate vocalizations. In Todt D., Goedeking P., Symmes D. (eds.), Primate Vocal Communication. Springer-Verlag, Berlin Heidelberg, pp 51-66.

Brown C. H., Gomez R., Waser P. M. 1995. Old world monkey vocalizations: adaptation to the local habitat? Animal Behaviour 50: 945-961.

Brown T. J., Handford P. 2000. Sound design for vocalizations: quality in the woods, consistency in the fields. The Condor 102: 81-92.

Brumm H. 2006. Signalling through acoustic windows: nightingales avoid interspecific competition by short-term adjustment of song timing. Journal of Comparative Physiology A 192: 1279-1285.

Brumm H., Voss K., Köllmer I., Todt D. 2004. Acoustic communication in noise: regulation of call characteristics in a New World monkey. Journal of Experimental Biology 207: 443-448.

Brumm H., Slabbekoorn H. 2005. Acoustic communication in noise. Advances in the Study of Behavior 35: 151-209.

Bulger J., Hamilton W. J. 1987. Rank and density correlates of inclusive fitness measures in a natural chacma baboon (Papio ursinus) troop. International Journal of Primatology 8: 635-650.

Buskirk W. H., Buskirk R. E., Hamilton III W. J. 1974. Troop-mobilizing behaviour of adult male chacma baboons. Folia Primatologica 22: 9-18.

Byrne R. W. 1981. Distance vocalizations of Guinea baboons (Papio papio) in Senegal: an analysis of function. Behaviour 78: 283-312.

Byrne R. W. 1982. Primate vocalizations: structural and functional approaches to understanding. Behaviour 78: 283-313.

Castles D. L., Whiten A., Aureli F. 1999. Social anxiety, relationships and self-directed behaviour among female olive baboons. Animal Behaviour 58: 1207-1215.

Chalmers N. R. 1980. Developmental relationships among social, manipulatory, postural and locomotor behaviours in olive baboons, Papio anubis. Behaviour 74: 22-37.

Chapman J. D., Chapman H. M. 2001. The Forest Flora of Taraba and Adamawa States, Nigeria. An Ecological Account and Plant Species Checklist. University of Canterbury, New Zealand.

Chapuis C. 1971. Un exemple de l'influence du milieu sur les émissions vocales des oiseaux: l'évolution des chants en forêt équatoriale. Terre et Vie 2: 183-202.

Cheney D. L., Seyfarth R. M. 1990. How Monkeys See the World. University of Chicago Press, Chicago. 
Cheney D. L., Seyfarth R. M., Silk J. B. 1995. The role of grunts in reconciling opponents and facilitating interactions among adult female baboons. Animal Behaviour 50: 249-257.

Cheney D. L., Seyfarth R. M., Palombit R. A. 1996. The function and mechanisms underlying baboon 'contact' barks. Animal Behaviour 52: 507-518.

Cheney D. L., Seyfarth R. M. 1997. Reconciliatory grunts by dominant female baboons influence victims' behaviour. Animal Behaviour 54: 409-418.

Cheney D. L., Seyfarth R. M., Fischer J., Beehner J., Bergman T., Johnson S. E., Kitchen D. M., Palombit R. A., Rendall D., Silk J. B. 2004. Factors affecting reproduction and mortality among baboons in the Okavango Delta, Botswana. International Journal of Primatology 25: 401-428.

Cheney D. L., Seyfarth R. M. 2007. Baboon Metaphysics: The Evolution of a Social Mind. University of Chicago Press, Chicago.

Chivers D. J. 1976. Communication within and between family groups of siamangs (Symphalangus syndactylus). Behaviour 57: 116-135.

Clack D. T. 1966. Effect of signal duration on the auditory sensitivity of humans and monkeys (Macaca mulatta). Journal of the Acoustical Society of America 40: 11401146.

Cosens S. E., Falls J. B. 1984. A comparison of sound propagation and song frequency in temperate marsh and grassland habitats. Behavioural Ecology and Sociobiology 15: 161-170.

Crockford C., Herbinger I., Vigilant L., Boesch C. 2004. Wild chimpanzees produce groupspecific calls: a case for vocal learning? Ethology 110: 221-243.

Dabelsteen T., Larsen O. N., Pedersen S. B. 1993. Habitat-induced degradation of sound signals: quantifying the effects of communication sounds and bird location on blur ratio, excess attenuation, and signal-to-noise ratio in blackbird song. Journal of the Acoustical Society of America 93: 2206-2220.

Dabelsteen T., Mathevon N. 2002. Why do songbirds sing intensively at dawn? A test of the acoustic transmission hypothesis. Acta Ethologica 4: 65-72.

Daniel J. C., Blumstein D. T. 1998. A test of the acoustic adaptation hypothesis in four species of marmots. Animal Behaviour 56: 1517-1528.

Date E. M., Lemon R. E. 1993. Sound transmission: a basis for dialects in birdsong? Behaviour 124: 291-312.

Davies J. G., Cowlishaw G. 1996. Baboon carnivory and raptor interspecific competition in the Namib Desert. Journal of Arid Environments 34: 247-249.

de Boer B. 2005. Evolution of speech and its acquisition. Adaptive Behavior 13: 281-292.

de la Torre S., Snowdon C. T. 2002. Environmental correlates of vocal communication of wild pygmy marmosets, Cebuella pygmaea. Animal Behaviour 63: 847-856.

de Vore I., Hall K. R. L. 1965. Baboon ecology. In de Vore I. (ed.), Primate Behaviour. Field Studies of Monkeys and Apes. Holt, Rinehart and Winston, New York, pp 20-52.

Delany M. E. 1977. Sound propagation in the atmosphere: a historical review. Acustica 38: 201-223.

Dooling R. 1979. Temporal summation in pure tones in birds. Journal of the Acoustical Society of America 65: 1058-1060. 
Dooling R. J., Searcy M. H. 1985. Temporal integration of acoustic signals by the budgerigar (Melopsittacus undulatus). Journal of the Acoustical Society of America 77: 19171920.

Doutrelant C., Lambrechts M. M. 2001. Macrogeographic variation in song - A test of competition and habitat effect in blue tits. Ethology 107: 533-544.

Dunbar R. I. M., Nathan M. F. 1972. Social organization of the Guinea baboon, Papio papio. Folia Primatologica 17: 321-334.

Dunn A. 1993. The Large Mammals of Gashaka Gumti National Park, Nigeria: Line Transect Surveys from Forest and Savannah. Report prepared for the Federal Ministry of Agriculture, Water Resources and Rural Development: Nigeria, NCF \& WWF-UK.

Eggeling W. J. 1947. Observations of the ecology of the Budongo rain forest, Uganda. Journal of Ecology 34: 20-87.

Egnor S. E. R., Hauser M. D. 2006. Noise-induced vocal modulation in cotton-top tamarins (Saguinus oedipus). American Journal of Primatology 68: 1183-1190.

Egnor S. E. R., Graham Wickelgren J., Hauser M. D. 2007. Tracking silence: adjusting vocal production to avoid acoustic interference. Journal of Comparative Physiological Psychology A 193: 477-483.

Elowson A. M., Snowdon C. T. 1994. Pygmy marmosets, Cebuella pygmaea, modify vocal structure in response to changed social environment. Animal Behaviour 47: 12671277.

Embleton T. F. W. 1963. Sound propagation in homogeneous deciduous and evergreen woods. Journal of the Acoustical Society of America 35: 1119-1125.

Endler J. A. 1992. Signals, signal conditions, and the direction of evolution. The American Naturalist 139: S125-S153.

Ey E., Pfefferle D., Fischer J. 2007a. Do age- and sex-related variations reliably reflect body size in non-human primate vocalizations? A review. Primates 48: 253-267.

Ey E., Hammerschmidt K., Seyfarth R. M., Fischer J. 2007b. Age- and sex-related variations in clear calls of Papio ursinus. International Journal of Primatology 28: 947-960.

Eyring C. 1946. Jungle acoustics. Journal of the Acoustical Society of America 18: 257-270.

Fischer J., Hammerschmidt K., Todt D. 1995. Factors affecting acoustic variation in Barbarymacaque (Macaca sylvanus) disturbance calls. Ethology 101: 51-66.

Fischer J., Hammerschmidt K., Todt D. 1998. Local variation in Barbary macaque shrill barks. Animal Behaviour 56: 623-629.

Fischer J., Hammerschmidt K., Cheney D. L., Seyfarth R. M. 2001a. Acoustic features of female chacma baboon barks. Ethology 107: 33-54.

Fischer J., Metz M., Cheney D. L., Seyfarth R. M. 2001b. Baboon responses to graded bark variants. Animal Behaviour 61: 925-931.

Fischer J., Hammerschmidt K., Cheney D. L., Seyfarth R. M. 2002. Acoustic features of male baboon loud calls: Influences of context, age, and individuality. Journal of the Acoustical Society of America 111: 1465-1474.

Fischer J., Kitchen D. M., Seyfarth R. M., Cheney D. L. 2004. Baboon loud calls advertise male quality: acoustic features and their relation to rank, age, and exhaustion. Behavioural Ecology and Sociobiology 56: 140-148. 
Fitch W. T. 2000. The evolution of speech: a comparative review. Trends in Cognitive Sciences 4: 258-267.

Fitch W. T. 2005. The evolution of language: a comparative review. Biology and Philosophy 20: $193-230$.

Fitch W. T., Hauser M. D. 1995. Vocal production in nonhuman primates: acoustics, physiology, and functional constraints on "honest" advertisement. American Journal of Primatology 37: 191-219.

Forrest T. G. 1994. From sender to receiver: propagation and environmental effects on acoustic signals. American Zoologist 34: 644-654.

Fotheringham J. R., Ratcliffe L. 1995. Song degradation and estimation of acoustic distance in black-capped chickadees (Parus atricapillus). Canadian Journal of Zoology 73: 858-868.

Fotheringham J. R., Martin P. R., Ratcliffe L. 1997. Song transmission and auditory perception of distance in wood warblers (Parulinae). Animal Behaviour 53: 12711285 .

Fricke F. 1984. Sound attenuation in forests. Journal of Sound and Vibration 92: 149-158.

Ganzhorn J. U. 2003. Habitat description and phenology. In Setchell J. M., Curtis D. J. (eds.), Field and Laboratory Methods in Primatology. Cambridge University Press, Cambridge, pp 40-56.

Ghazanfar A. A. 2008. Language evolution: neural differences that make a difference. Nature Neuroscience 11: 382-384.

Ghazanfar A. A., Hauser M. D. 1999. The neuroethology of primate vocal communication: substrates for the evolution of speech. Trends in Cognitive Sciences 3: 377-384.

Ghazanfar A. A., Rendall D. 2008. Evolution of human vocal production. Current Biology 18: R457-R460.

Gilmore H. A. 1978. Message analysis of a baboon vocalization. American Journal of Physical Anthropology 48: 398-398.

Gilmore H. A. 1979. Function of the female post-copulatory vocalization in olive baboons. American Journal of Physical Anthropology 50: 442-442.

Gilmore H. A. 1983a. Approaching others: a functional analysis of the grunt vocalization among olive baboons. American Journal of Primatology 4: 343-343.

Gilmore H. A. 1983b. Grunting and groaning: rethinking the baboon contact call. American Journal of Physical Anthropology 60: 197-197.

Gish S. L., Morton E. S. 1981. Structural adaptations to local habitat acoustics in Carolina wren songs. Zeitschrift für Tierpsychologie 56: 74-84.

Hage S. R. 2005. Audiovokale Integration und vokalmotorische Kontrolle im pontinen Hirnstamm - Telemetrische Einzelzellableitungen am sich frei bewegenden Totenkopfaffen. PhD thesis. Fakultät für Wissenschaft der Universität Ulm, Germany.

Hall K. R. L. 1963. Variations in the ecology of the chacma baboon, Papio ursinus. Symposium of the Zoological Society London 10: 1-28.

Hall K. R. L., de Vore I. 1965. Baboon social behavior. In de Vore I. (ed.), Primate Behaviour. Field Studies of Monkeys and Apes. Holt, Rinehart, Winston, New York, pp 53-110. 
Hammerschmidt K., Newman J. D., Champoux M., Suomi S. J. 2000. Changes in rhesus macaques 'coo' vocalizations during early development. Ethology 106: 873-886.

Hammerschmidt K., Freudenstein T., Jürgens U. 2001. Vocal development in squirrel monkeys. Behaviour 138: 1179-1204.

Hammerschmidt K., Fischer J. 2008. Constraints in primate vocal production. In Griebel U., Oller K. (eds.), The Evolution of Communicative Creativity: From Fixed Signals to Contextual Flexibility. The MIT Press, Cambridge, Massachusetts.

Handford P. 1981. Vegetational correlates of variation in the song of Zonotrichia capensis. Behavioural Ecology and Sociobiology 8: 203-206.

Handford P. 1988. Trill rate dialects in the rufous-collared sparrow, Zonotrichia capensis, in northwestern Argentina. Canadian Journal of Zoology 66: 2658-2670.

Handford P., Lougheed S. C. 1991. Variation in duration and frequency characters in the song of the rufous-collared sparrow, Zonotrichia capensis, with respect to habitat, trill dialects and body size. The Condor 93: 644-958.

Hansen P. 1979. Vocal learning: its role in adapting sound structures to long-distance propagation, and a hypothesis on its evolution. Animal Behaviour 27: 1270-1271.

Harcourt C. S., Ellerton N. G. 1995. A Brief Look at the Primates of GGNP, Nigeria. Report prepared for the WWF-UK and NCF.

Harding R. S. O. 1976. Ranging patterns of a troop of baboons (Papio anubis) in Kenya. Folia Primatologica 25: 143-185.

Hauser M. D. 1996. The Evolution of Communication. The MIT Press, Cambridge, Massachusetts.

Hayes K. J., Nissen C. H. 1971. Higher mental functions of a home-raised chimpanzee. In Schrier A. M., Stollnitz F. (eds.), Behaviour of Non-human Primates 4. Academic Press, New York, pp 50-115.

Heffner H. E. 1998. Auditory awareness. Applied Animal Behaviour Science 57: 259-268.

Heffner R. S. 2004. Primate hearing from a mammalian perspective. The Anatomical Record Part A 281A: 1111-1122.

Henwood K., Fabrick A. 1979. A quantitative analysis of the dawn chorus: temporal selection for communicatory optimization. The American Naturalist 114: 260-274.

Henzi P., Barrett L. 2003. Evolutionary ecology, sexual conflict, and behavioral differentiation among baboon populations. Evolutionary Anthropology 12: 217-230.

Higham J. P. 2006. The Reproductive Ecology of Female Olive Baboons (Papio hamadryas anubis) at Gashaka-Gumti National Park, Nigeria. PhD thesis. School of Human and Life Sciences, University of Surrey Roehampton, United Kingdom.

Higham J. P., Ross C., Warren Y, Heistermann M., MacLarnon A. M. 2007. Reduced reproductive function in wild baboons (Papio hamadryas anubis) related to natural consumption of the African black plum (Vitex doniana). Hormones and Behaviour 52: 384-390.

Higham J. P., MacLarnon A. M., Ross C., Heistermann M., Semple S. 2008. Baboon sexual swellings: information content of size and color. Hormones and Behavior 53: 452-462.

Hill R. A., Lycett J. E., Dunbar R. I. M. 2000. Ecological and social determinants of birth intervals in baboons. Behavioral Ecology 11: 560-564. 
Hill R. A., Dunbar R. I. M. 2002. Climatic determinants of diet and foraging behaviour in baboons. Evolutionary Ecology 16: 579-593.

Hill R. A., Barrett L., Gaynor D., Weingrill T., Dixon P., Payne H., Henzi S. P. 2003. Day length, latitude and behavioural (in)flexibility in baboons (Papio cynocephalus ursinus). Behavioural Ecology and Sociobiology 53: 278-286.

Howard P. C. 1991. Nature Conservation in Uganda's Tropical Forest Reserves. Gland, I.U.C.N.

Howard P. C., Davenport T., Kigenyi F. 1997. Planning conservation areas in Uganda's natural forests. Oryx 31: 253-264.

Hunter M. L. Jr. 1980. Microhabitat selection for singing and other behaviour in great tit, Parus major: some visual and acoustical considerations. Animal Behaviour 28: 468475.

Hunter M. L. Jr., Krebs J. R. 1979. Geographical variation in the song of the great tit (Parus major) in relation to ecological factors. Journal of Animal Ecology 48: 759-785.

Hylton R., Godard R. D. 2001. Song properties of indigo buntings in open and forested habitats. The Wilson Bulletin 113: 243-245.

Ingard U. 1953. A review of the influence of meteorological conditions on sound propagation. Journal of the Acoustical Society of America 25: 405-411.

James F. C., Shugart H. H. Jr. 1970. A quantitative method of habitat description. Audubon Field Notes 24: 727-736.

Janik V. M. 2000. Whistle matching in wild bottlenose dolphins (Tursiops truncatus). Science 289: 1355-1357.

Janik V. M., Slater P. J. B. 1997. Vocal learning in mammals. Advances in the Study of Behavior 26: 59-99.

Janik V. M., Slater P. J. B. 2000. The different roles of social learning in vocal communication. Animal Behaviour 60: 1-11.

Jenkins P. F., Baker A. J. 1984. Mechanisms of song differentiation in introduced populations of chaffinches Fringilla coelebs in New Zealand. Ibis 126: 510-524.

Jilka A., Leisler B. 1974. Die Einpassung dreier Rohrsängerarten (Acrocephalus schoenobaenus, A. scirpaceus, A. arundinaceus) in ihre Lebensräume in Bezug auf das Frequenzspektrum ihrer Reviergesänge. Journal für Ornithologie 115: 192-212.

Jürgens U. 2002. Neural pathways underlying vocal control. Neuroscience and Behavioral Reviews 26: 235-258.

Jürgens U., Ploog D. 1981. On the neural control of mammalian vocalization. Trends in Neurosciences 4: 135-137.

Kacelnik A., Krebs J. R. 1983. The dawn chorus in the great tit (Parus major): proximate and ultimate causes. Behaviour 83: 287-308.

Kalko E. K. V. 1995. Echolocation signal design, foraging habitats and guild structure in six Neotropical sheath-tailed bats (Emballonuridae). Symposium of the Zoological Society London 67: 259-273.

Kalko E. K. V., Schnitzler H.-U. 1993. Plasticity in echolocation signals of European pipistrelle bats in search flight: implications for habitat use and prey detection. Behavioral Ecology and Sociobiology 33: 415-428. 
Kappeler P. M. 2000. Grouping and movement patterns of Malagasy primates. In Boinski S., Garber P. (eds.), On the Move: How and Why Animals Travel in Groups. The University of Chicago Press, Chicago, pp 470-490.

Kime N. M., Turner W. R., Ryan M. J. 2000. The transmission of advertisement calls in Central American frogs. Behavioral Ecology 11: 71-83.

Kingdon J. 1997. The Kingdon Field Guide to African Mammals. Academic Press, San Diego.

Kitchen D. M., Cheney D. L., Seyfarth R. M. 2003a. Female baboons' responses to male loud calls. Ethology 109: 401-412.

Kitchen D. M., Seyfarth R. M., Fischer J., Cheney D. L. 2003b. Loud calls as indicators of dominance in male baboons (Papio cynocephalus ursinus). Behavioural Ecology and Sociobiology 53: 374-384.

Kitchen D. M., Cheney D. L., Seyfarth R. M. 2005. Male chacma baboons (Papio cynocephalus ursinus) discriminate loud call contests between rivals of different relative ranks. Animal Cognition 8: 1-6.

Klump G. M., Maier E. H. 1990. Temporal summation in the European starling (Sturnus vulgaris). Journal of Comparative Psychology 104: 94-100.

Koda H. 2004. Flexibility and context-sensitivity during the vocal exchange of coo calls in wild Japanese macaques (Macaca fuscatat yakui). Behaviour 141: 1279-1296.

Koda H., Shimooka Y., Sugiura H. 2008. Effect of caller activity and habitat visibility on contact call rate of wild Japanese macaques (Macaca fuscata). American Journal of Primatology 70: 1-9.

Kopuchian C., Lijtmaer D. A., Tubaro P. L., Handford P. 2004. Temporal stability and change in a microgeographical pattern of song variation in the rufous-collared sparrow. Animal Behaviour 68: 551-559.

Kummer H. 1968. Social Organization of Hamadryas Baboons. A Field Study. Karger, Basel.

Kunz B. K., Linsenmair K. E. 2008a. The disregarded West: diet and behavioural ecology of olive baboons in the Ivory Coast. Folia Primatologica 79: 31-51.

Kunz B. K., Linsenmair K. E. 2008b. The role of the olive baboon (Papio anubis, Cercopithecidae) as seed disperser in a savannah-forest mosaic of West Africa. Journal of Tropical Ecology 24: 235-246.

Lameira A. R., Wich S. A. 2008. Orangutans long call degradation and individuality over distance: a playback approach. International Journal of Primatology 29: 615-625.

Lemasson A., Gautier J.-P., Hausberger M. 2003. Vocal similarities and social bonds in Campbell's monkey (Cercopithecus campbelli). Comptes Rendus Biologies 326: 1185-1193.

Lemasson A., Hausberger M. 2004. Patterns of vocal sharing and social dynamics in a captive group of Campbell's monkeys (Cercopithecus campbelli campbelli). Journal of Comparative Psychology 118: 347-359.

Lemasson A., Palombit R. A., Jubin R. 2008. Friendships between males and lactating females in a free-ranging group of olive baboons (Papio hamadryas anubis): evidence from playback experiments. Behavioral Ecology and Sociobiology 62: 1027-1035. 
Lemon R. E., Struger J., Lechowicz M. J., Norman R. F. 1981. Song features and singing heights of American warblers: maximization or optimization of distance? Journal of the Acoustical Society of America 69: 1169-1176.

Leonard M. L., Horn A. G. 2005. Ambient noise and the design of begging signals. Proceedings of the Royal Society of London Series B 272: 651-656.

Lieberman P. 2002. On the nature and evolution of the neural bases of human language. Yearbook of Physical Anthropology 45: 36-62.

Lijtmaer D. A., Tubaro P. L. 2007. A reversed pattern of association between song dialects and habitat in the rufous-collared sparrow. The Condor 109: 658-667.

Linskens H. F., Martens M. J. M., Hendriksen H. J. G. M., Roestenbergs-Sinnige A. M., Brouwers W. A. J. M., van der Staak A. L. H. C., Strik-Jansen A. M. J. 1976. The acoustic climate of plant communities. Oecologia 23: 165-177.

Lombard E. 1911. Le signe de l'élévation de la voix. Annales des Maladies de l'Oreille et du Larynx 37: 101-119.

MacLarnon A., Hewitt G. P. 1999. The evolution of human speech: the role of enhanced breathing control. American Journal of Physical Anthropology 109: 341-363.

Macpherson E. A., Middlebrooks J. C. 2000. Localization of brief sounds: effects of level and background noise. Journal of the Acoustical Society of America 108: 1834-1849.

Maestripieri D., Leoni M., Raza S. S., Hirsch E. J., Whitham J. C. 2005. Female copulation calls in Guinea baboons: evidence for postcopulatory female choice? International Journal of Primatology 26: 737-758.

Maestripieri D., Mayhew J., Carlson C. L., Hoffman C. L., Radtke J. M. 2007. One-male harems and female social dynamics in Guinea baboons. Folia Primatologica 78: 56-68.

Marler P. 1970. Birdsong and speech development: could there be parallels? American Scientist 58: 669-673.

Marler P. M. 1974. Animal communication. In Krames L., Pliner P., Alloway T. (eds.), Nonverbal Communication. Plenum Press, New York, pp 25-50.

Marler P. M. 1977. The evolution of communication. In Sebeok T. A. (ed.), How Animal Communicate I. Indiana University Press, Bloomington \& London, pp 45-70.

Marten K., Marler P. M. 1977. Sound transmission and its significance for animal vocalization. I. Temperate habitats. Behavioural Ecology and Sociobiology 2: 271290.

Marten K., Quine D., Marler P. M. 1977. Sound transmission and its significance for animal vocalization. II. Tropical forest habitats. Behavioural Ecology and Sociobiology 2: 291-302.

Masataka N., Symmes D. 1986. Effect of separation distance on isolation call structure in squirrel monkeys (Saimiri sciureus). American Journal of Primatology 10: 271-278.

Masters J. C. 1991. Loud calls of Galago crassicaudatus and G. garnettii and their relation to habitat structure. Primates 32: 153-167.

Mathevon N., Aubin T., Dabelsteen T. 1996. Song degradation during propagation: importance of song post for the wren Troglodytes troglodytes. Ethology 102: 397-412. 
Mays Jr. H. L., Yao C.-T., Yuan H.-W. 2006. Antiphonal duetting in Steere's liocichla (Liocichla steerii): male song individuality and correlation between habitat and duetting behavior. Ecological Research 21: 311-314.

McCracken K. G., Sheldon F. H. 1997. Avian vocalizations and phylogenetic signal. Proceedings of the National Academy of Science USA 94: 3833-3836.

Meise K. 2008. Information Content of Female Chacma Baboon Grunts - The Signallers' Potential to Utter Functionally Referential Grunts. Diploma thesis. University of Bielefeld, Germany.

Meyer J. 2004. Bioacoustics of human whistled languages: an alternative approach to the cognitive processes of language. Anais da Academia Brasileira de Ciências 76: 405412.

Meyer J. 2005. Description Typologique et Intelligibilité des Langues Sifflées, Approche Linguistique et Bioacoustique. Université Lumière Lyon 2, France.

Mitani J. C., Stuht J. 1998. The evolution of nonhuman primate loud calls: acoustic adaptation for long-distance transmission. Primates 39: 171-182.

Mitani J. C., Hunley K. L., Murdoch M. E. 1999. Geographic variation in the calls of wild chimpanzees: a reassessment. American Journal of Primatology 47: 133-151.

Morton E. S. 1975. Ecological sources of selection on avian sounds. The American Naturalist 109: 17-34.

Morton E. S. 1986. Predictions from the ranging hypothesis for the evolution of long-distance signals in birds. Behaviour 99: 65-86.

Mundry R., Fischer J. 1998. Use of statistical programs for nonparametric tests of small samples often leads to incorrect P values: examples from Animal Behaviour. Animal Behaviour 56: 256-259.

Naguib M. 2003. Reverberation of rapid and slow trills: implications for signal adaptations to long-range communication. Journal of the Acoustical Society of America 113: 17491756.

Naguib M., Wiley R. H. 2001. Estimating the distance to a source of sound: mechanisms and adaptations for long-range communication. Animal Behaviour 62: 825-837.

Naguib M., Hammerschmidt K., Wirth J. 2001. Microgeographic variation, habitat effects and individual signature cues in calls of chiffchaffs Phylloscopus collybita canarensis. Ethology 107: 341-355.

Nelson B. S. 2003. Reliability of sound attenuation in Florida scrub habitat and behavioural implications. Journal of the Acoustical Society of America 113: 2901-2911.

Nemeth E., Winkler H., Dabelsteen T. 2001. Differential degradation of antbird songs in a Neotropical rainforest: adaptation to perch height? Journal of the Acoustical Society of America 110: 3263-3274.

Nemeth E., Dabelsteen T., Pedersen S. B., Winkler H. 2006. Rainforests as concert halls for birds: are reverberations improving sound transmission of long song elements? Journal of the Acoustical Society of America 119: 620-626.

Newman T. K., Jolly C. J., Rogers J. 2004. Mitochondrial phylogeny and systematics of baboons. American Journal of Physical Anthropology 124: 17-27.

Nicholls J. A., Goldizen A. W. 2006. Habitat type and density influence vocal design in satin bowerbirds. Journal of Animal Ecology 75: 549-558. 
Nottebohm F. 1975. Continental patterns of song variability in Zonotrichia capensis: some possible ecological correlates. The American Naturalist 109: 605-624.

O'Connell S. M., Cowlishaw G. 1994. Infanticide avoidance, sperm competition and mate choice: the function of copulation calls in female baboons. Animal Behaviour 48: 687694.

Oda R. 1996. Effects of contextual and social variables on contact call production in free ranging ringtailed lemurs (Lemur catta). International Journal of Primatology 17: 191205.

Oda R. 2002. Individual distinctiveness of the contact calls of ring-tailed lemurs. Folia Primatologica 73: 132-136.

Odendaal F. J., Bull C. M., Telford S. R. 1986. Influence of the acoustic environment on the distribution of the frog Ranidella riparia. Animal Behaviour 34: 1836-1843.

Owren M. J., Seyfarth R. M., Cheney D. L. 1997. The acoustic features of vowel-like grunt calls in chacma baboons (Papio cynocephalus ursinus): implications for production processes and functions. Journal of the Acoustical Society of America 101: 29512963.

Padgham M. 2004. Reverberation and frequency attenuation in forests - Implications for acoustic communication. Journal of the Acoustical Society of America 115: 402-410.

Palombit R. A., Seyfarth R. M., Cheney D. L. 1997. The adaptive value of "friendships" to female baboons: experimental and observational evidence. Animal Behaviour 54: 599614.

Palombit R. A., Cheney D. L., Seyfarth R. M. 1999. Male grunts as mediators of social interaction with females in wild chacma baboons (Papio cynocephalus ursinus). Behaviour 136: 221-242.

Palombit R. A., Cheney D. L., Seyfarth R. M. 2001. Female-female competition for male 'friends' in wild chacma baboons, Papio cynocephalus ursinus. Animal Behaviour 61: 1159-1171.

Parris K. M. 2002. More bang for your buck: the effect of caller position, habitat and chorus noise on the efficiency of calling in the spring peeper. Ecological Modelling 156: 213224.

Payne R. B. 1978. Microgeographic variation in songs of splendid sunbirds Nectarinia coccinigaster: population phenetics, habitats, and song dialects. Behaviour 65: 282308.

Paterson J. D. 1991. The Ecology and History of Uganda's Budongo Forest. Forest and Conservation History 35: 179-187.

Penna M., Solis R. 1998. Frog call intensities and sound propagation in the South American temperate forest region. Behavioral Ecology and Sociobiology 42: 371-381.

Perla B. S., Slobodchikoff C. N. 2002. Habitat structure and alarm call dialects in Gunnison's prairie dog (Cynomys gunnisoni). Behavioral Ecology 13: 844-850.

Peters G., Baum L., Peters M. K., Tonkin-Leyhausen B. 2008. Spectral characteristics of intense mew calls in cat species of the genus Felis (Mammalia: Carnivora: Felidae). Journal of Ethology DOI 10.1007/s10164-008-0107-y.

Pfefferle D., Fischer J. 2006. Sound and size: identification of acoustic variables that reflect body size in hamadryas baboons, Papio hamadryas. Animal Behaviour 72: 43-51. 
Pierce J. D. 1985. A review of attempts to condition operantly alloprimate vocalizations. Primates 26: 202-213.

Piercy J. E., Embleton T. F. W., Sutherland L. C. 1977. Review of noise propagation in the atmosphere. Journal of the Acoustical Society of America 61: 1403-1418.

Ploog D. 2002. Is the neural basis of vocalization different in non-human primates and Homo sapiens? In Crow T. J. (ed.), The Speciation of Modern Homo sapiens. Oxford University Press, Oxford, pp 121-135.

Plumptre A. J., Reynolds V., Bakuneeta C. 1994. The Contribution of Fruit-eating Primates to Seed Dispersal and Natural Regeneration after Selective Logging. Unpublished report to ODA project no. R 4738.

Potash L. M. 1972. Noise-induced changes in calls of the Japanese quail. Psychonomic Science 26: 252-254.

Price M. A., Attenborough K., Heap N. W. 1988. Sound attenuation through trees: measurements and models. Journal of the Acoustical Society of America 84: 18361844.

Rahn C. 2008. Usage, Structure and Function of Olive Baboon Contact Calls. A Field Study in Budongo Forest, Uganda. Diploma thesis. Free University Berlin, Germany.

Ralls K., Fiorelli P., Gish S. 1985. Vocalizations and vocal mimicry in captive harbor seals, Phoca vitulina. Canadian Journal of Zoology 63: 1050-1056.

Ransom T. W. 1981. Beach Troop of the Gombe. Associated University Press Inc, Lewisburg.

Rendall D. 2003. Acoustic correlates of caller identity and affect intensity in the vowel-like grunt vocalizations of baboons. Journal of the Acoustical Society of America 113: 3390-3402.

Rendall D., Seyfarth R. M., Cheney D. L., Owren M. J. 1999. The meaning and function of grunt variants in baboons. Animal Behaviour 57: 583-592.

Rendall D., Cheney D. L., Seyfarth R. M. 2000. Proximate factors mediating "contact" calls in adult female baboons (Papio cynocephalus ursinus) and their infants. Journal of Comparative Psychology 114: 36-46.

Rendall D., Owren M. J. 2002. Animal vocal communication: Say what? In Bekoff M., Allen C. A., Burghardt G. (eds.), The Cognitive Animal. Empirical and Theoretical Perspectives in Animal Cognition. The MIT Press, Cambridge, Massachusetts, pp 307-313.

Rendall D., Owren M. J., Weerts E., Hienz R. D. 2004. Sex differences in the acoustic structure of vowel-like grunt vocalizations in baboons and their perceptual discrimination by baboon listeners. Journal of the Acoustical Society of America 115: 411-421.

Richards D. G., Wiley R. H. 1980. Reverberation and amplitude fluctuations in the propagation of sound in a forest: implications for animal communication. The American Naturalist 115: 381-399.

Robinson J. G. 1982. Vocal systems regulating within-group spacing. In Snowdon C. T., Brown C. H., Petersen M. R. (eds.), Primate Communication. Cambridge University Press, Cambridge, pp 94-116.

Ron T., Henzi S. P., Motro U. 1996. Do female chacma baboons compete for a safe spatial position in a southern woodland habitat? Behaviour 133: 475-490. 
Rowell T. E. 1966. Forest living baboons in Uganda. Journal of Zoology of London 149: 344364.

Rowell T. 1972. Social Behaviour of Monkeys. Penguin Books, New York.

Ryan M. J., Brenovitz E. A. 1985. The role of body size, phylogeny, and ambient noise in the evolution of bird song. The American Naturalist 126: 87-100.

Ryan M. J., Cocroft R. B., Wilczynski W. 1990. The role of environmental selection in intraspecific divergence of mate recognition signals in the cricket frog, Acris crepitans. Evolution 44: 1869-1872.

Sarmiento E. 1997. Current problems with Papio taxanomies. African Primates 3: 48-52.

Saunders J., Slotow R. 2004. The evolution of song structure in southern African birds: an assessment of the acoustic adaptation hypothesis. Ostrich 75: 147-155.

Schneider C., Hodges K., Fischer J., Hammerschmidt K. 2008. Acoustic niches of Siberut primates. International Journal of Primatology 29: 601-613.

Schrader L., Hammerschmidt K. 1997. Computer-aided analysis of acoustic parameters in animal vocalizations: a multi-parametric approach. Bioacoustics 7: 247-265.

Schusterman R. J., Reichmuth C. 2008. Novel sound production through contingency learning in the Pacific walrus (Odobenus rosmarus divergens). Animal Cognition 11: 319-327.

Seddon N. 2005. Ecological adaptation and species recognition drives vocal evolution in neotropical suboscine birds. Evolution 59: 200-215.

Semple S., McComb K., Alberts S., Altmann J. 2002. Information content of female copulation calls in yellow baboons. American Journal of Primatology 56: 43-56.

Seyfarth R. M., Cheney D. L. 1986. Vocal development in vervet monkeys. Animal Behaviour 34: 1640-1658.

Seyfarth R. M., Cheney D. L. 1997. Some general features of vocal development in nonhuman primates. In Snowdon C. T., Hausberger M. (eds.), Social Influences on Vocal Development. Cambridge University Press, Cambridge, pp 249-273.

Shy E. 1983. The relation of geographical variation in song to habitat characteristics and body size in North American tanagers (Thraupinae: Piranga). Behavioural Ecology and Sociobiology 12: 71-76.

Shy E., Morton E. S. 1986. Adaptation of amplitude structure of songs to propagation in field habitat in song sparrows. Ethology 72: 177-184.

Siemers B. M., Kalko E. K. V., Schnitzler H.-U. 2001. Echolocation behavior and signal plasticity in the Neotropical bat Myotis nigricans (Schinz, 1821) (Vespertilionidae): a convergent case with European species of Pipistrellus? Behavioral Ecology and Sociobiology 50: 317-328.

Silk J. B., Cheney D. L., Seyfarth R. M. 1996. The form and function of post-conflict interactions between female baboons. Animal Behaviour 52: 259-268.

Sinnott J. M., Stebbins W. C., Moody D. B. 1975. Regulation of voice amplitude by the monkey. Journal of the Acoustical Society of America 58: 412-414.

Slabbekoorn H. 2004. Habitat-dependent ambient noise: consistent spectral profiles in two African forest types. Journal of the Acoustical Society of America 116: 3727-3733. 
Slabbekoorn H., Smith T. B. 2002. Habitat-dependent song divergence in the little greenbul: an analysis of environmental selection pressures on acoustic signals. Evolution 56: 1849-1858.

Slabbekoorn H., Peet M. 2003. Birds sing at a higher pitch in urban noise. Nature 424: 267267.

Slabbekoorn H., den Boer-Visser A. 2006. Cities change the songs of birds. Current Biology 16: $2326-2331$.

Slabbekoorn H., Yeh P., Hunt K. 2007. Song transmission and song divergence: a comparison of urban and forest acoustics. The Condor 109: 67-78.

Smith J. I., Yu H. T. 1992. The association between vocal characteristics and habitat type in Taiwanese passerines. Zoological Science 9: 659-664.

Smith R. J., Jungers W. L. 1997. Body mass in comparative primatology. Journal of Human Evolution 32: 523-559.

Snowdon C. T., Cleveland J. 1980. Individual recognition of contact calls by pygmy marmosets, Cebuella pygmaea. Animal Behaviour 28: 717-727.

Snowdon C. T., de la Torre S. 2002. Multiple environment contexts and communication in pygmy marmosets (Cebuella pygmaea). Journal of Comparative Psychology 116: 182188.

Sorjonen J. 1986a. Song structure and singing strategies in the genus Luscinia in different habitats and geographical areas. Behaviour 98: 274-285.

Sorjonen J. 1986b. Factors affecting the structure of song and the singing behaviour of some northern European passerine birds. Behaviour 98: 286-304.

Strum S. C. 1987. Almost Human. A Journey into the World of Baboons. Random House, New York.

Strum S. C. 1991. Weight and age in wild olive baboons. American Journal of Primatology 25: 219-237.

Stueckle S., Zinner D. 2008. To follow or not to follow: decision making and leadership during the morning departure in chacma baboons. Animal Behaviour 75: 1995-2004.

Sugiura H. 2007. Effects of proximity and behavioural context on acoustic variation in the coo calls of Japanese macaques. American Journal of Primatology 69: 1-13.

Sugiura H., Tanaka T., Masataka N. 2006. Sound transmission in the habitats of Japanese macaques and its possible effect on population differences in coo calls. Behaviour 143: 993-1012.

Sutton D., Larson C., Taylor E. M., Lindeman R. C. 1973. Vocalizations in rhesus monkeys: conditionability. Brain Research 52: 225-231.

Sutton D., Larson C., Lindeman R. C. 1974. Neocortical and limbic lesion effects on primate phonation. Brain Research 71: 61-75.

Todt D. 2004. From birdsong to speech: a plea for comparative approaches. Anais da Academia Brasileira de Ciências 76: 201-208.

Tubaro P. L., Segura E. T., Handford P. 1993. Geographic variation in the song of the rufouscollared sparrow in eastern Argentina. The Condor 95: 588-595. 
Tubaro P. L., Segura E. T. 1994. Dialect differences in the song of Zonotrichia capensis in the southern pampas: a test of the acoustic adaptation hypothesis. The Condor 96: 10841088 .

Tubaro P. L., Mahler B. 1998. Acoustic frequencies and body mass in New World doves. The Condor 100: 54-61.

Tubaro P. L., Lijtmaer D. A. 2006. Environmental correlates of song structure in forest grosbeaks and saltators. The Condor 108: 120-129.

Tuttle M. D., Ryan M. J. 1982. The role of synchronized calling, ambient light, and ambient noise in anti-bat-predator behavior of a treefrog. Behavioral Ecology and Sociobiology 11: $125-131$.

van Buskirk J. 1997. Independent evolution of song structure and note structure in American wood warblers. Proceedings of the Royal Society of London Series B 264: 755-761.

van Schaik C. P. 1983. Why are diurnal primates living in groups? Behaviour 87: 120-144.

Vick S.-J., Anderson J. R. 2003. Use of human visual attention cues by olive baboons (Papio anubis) in a competitive task. Journal of Comparative Psychology 117: 209-216.

Waas J. R. 1988. Song pitch - habitat relationships in white-throated sparrows: cracks in acoustic windows? Canadian Journal of Zoology 66: 2578-2581.

Warren Y. 2003. Olive Baboons (Papio cynocephalus anubis): Behaviour, Ecology and Human Conflict in Gashaka Gumti National Park, Nigeria. PhD thesis. School of Life and Sports Sciences, University of Surrey Roehampton, United Kingdom.

Waser P. M. 1977. Individual recognition, intra-group cohesion and inter-group spacing: evidence from sound playback to forest monkeys. Behaviour 60: 28-74.

Waser P. M. 1982. The evolution of male loud calls among mangabeys and baboons. In Snowdon C. T., Brown C. H., Petersen M. R. (eds.), Primate Communication. Cambridge University Press, Cambridge, pp 117-143.

Waser P. M., Waser M. S. 1977. Experimental studies of primate vocalization: specializations for long-distance propagation. Zeitschrift für Tierpsychologie 43: 239-263.

Waser P. M., Brown C. H. 1984. Is there a "sound window" for primate communication? Behavioural Ecology and Sociobiology 15: 73-76.

Waser P. M., Brown C. H. 1986. Habitat acoustics and primate communication. American Journal of Primatology 10: 135-154.

Wasserman F. E. 1979. The relationship between habitat and song in the white-throated sparrow. Condor 81: 424-426.

Westfall P. H., Young S. S. 1993. Resampling-based multiple testing. Examples and methods for p-value adjustments. Wiley J., New York.

Wiley R. H. 1991. Associations of song properties with habitats for territorial oscine birds of Eastern North America. The American Naturalist 138: 973-993.

Wiley R. H., Richards D. G. 1978. Physical constraints on acoustic communication in the atmosphere: implications for the evolution of animal vocalizations. Behavioural Ecology and Sociobiology 3: 69-94.

Williams J. M., Slater P. J. B. 1993. Does chaffinch Fringilla coelebs song vary with the habitat in which it is sung? Ibis 135: 202-208. 
Wind J., Chiarelly B., Bichakjian B., Nocentini B. 1992. Language Origin: A Multidisciplinary Approach. Kluwer Academic Publishers, Dordrecht.

Winter P., Handley P., Ploog D., Schott D. 1973. Ontogeny of squirrel monkey calls under normal conditions and under acoustic isolation. Behaviour 43: 230-239.

Wund M. A. 2005. Learning and the development of habitat-specific bat echolocation. Animal Behaviour 70: 441-450.

Zimmermann B. L. 1983. A comparison of structural features of calls of open and forest habitat frog species in the Central Amazon. Herpetologica 39: 235-246.

Zinner D., Groeneveld L. F., Keller C., Roos C. 2008. Multiple mtDNA paraphylies in baboons (Papio spp.) and their biogeographical and taxonomical implications. Submitted to BMC Evolutionary Biology.

Zinner D., Buba U., Roos C. In press. Out of Africa and slightly beyond. Phylo-geography of baboons. In Sommer V., Ross C. (eds.), Primates of Gashaka. Socio-ecology and Conservation in Nigeria's Biodiversity Hotspot. Series: Developments in Primatology: Progress and Prospects. Springer, New York.

Zuberbühler K., Noe R., Seyfarth R. M. 1997. Diana monkey long-distance calls: messages for conspecifics and predators. Animal Behaviour 53: 589-604. 


\section{Acknowledgements}

The work upon which this thesis is based would not have been possible without the collaboration of many people and institutions. Institutional support was provided by the German Primate Centre. Financial support was provided by the German Academic Exchange Service (Deutsches Akademisches Austauschsdienst) and the German Primate Centre.

First of all, I would like to thank my supervisor Julia Fischer for giving me the opportunity to join her stimulating team. I am greatly indebted to her for all what I have learnt during this time and for her support. Her constructive criticisms helped me to build my own critical sense and encouraged me to improve my arguments.

I am very grateful to Kurt Hammerschmidt for his patience when explaining me acoustic and statistical analyses. Discussions with him helped me to rethink and improve many parts of the thesis.

Field work in Gashaka-Gumti National Park benefited from a permit by the Nigerian National Parks Service to the Gashaka Primate Project (GPP), which receives its core funding from the Chester Zoo Nigeria Biodiversity Programme. I would like to thank Volker Sommer and Caroline Ross for allowing me to join GPP.

I am greatly indebted to all the people who helped in the field. Special thanks go to my field assistants Halidu Ilyusun, Mustapha Isa, Abdulkadiri Adamu and Haruna Ahmadu. Their ability to find the baboons and to follow one particular individual in dense grass still astonished me after several months working with them. Their patience and dedication facilitated data collection. Felix Vitalis and Maikanti Hassan cared for cooking and daily life in Kwano. Musa Basago, the keeper of GPP house in Gashaka, introduced me to the Nigerian life and made it much smoother for me. He largely contributed to the unforgettable time I had in Nigeria. Daphné Kerhoas provided companionship in the field and helped for sine waves broadcasts for habitat characterisation.

I would like to thank all the people of the department of Cognitive Ethology in the German Primate Centre for helpful comments and discussions, and also just for being there when I came out of my cellar.

I am grateful to Ellen Wiese and to people from the "Personal Büro" for helping me to go through the German administration. 
Many thanks go to Dana Pfefferle and Charlotte Rahn who constituted the major actors of my social life in Göttingen and the nice staff of my own "emergency service" for German paperwork. Both provided friendship and support, and also invested a substantial amount of time in reading earlier versions of this thesis, which greatly benefited from their comments and suggestions. Charlotte kindly provided data collected in Uganda for her diploma thesis and helped in their analyses.

I would like to thank my "far-from-eyes-but-not-from-the-heart" friends who made this period even more enjoyable: Aude, Carine, David, Myriam, Petite Sophie, Sandrine and Sophsouris. Thank you so much for being there, encouraging me, bearing my anxiousness, laughing at my 2-cents jokes, as well as reminding me from time to time to keep my mind open for other things in life than a thesis.

Special thanks go to Vincent who provided much more than logistical support in the field and back in Germany. Thank you for having been what you were and for having contributed to the best days of my life.

So many thanks should go to the members of my family who supported me during my whole life, and shared all ups and downs of this thesis. They coped patiently with their anxiety while I was enjoying daily life with the baboons who allowed me to stay with them. I am especially grateful to my parents and my brother who are always present when I need any support... and even before I notice that I need anything. Without their dedication and encouragement, I would be neither where I am nor who I am, now. 


\section{Appendices}

Appendix 1: Synthetic view of studies examining environmental influences on usage of vocal signals in (a) birds and (b) anurans and mammals.

\begin{tabular}{|c|c|c|c|}
\hline Study & Species & $\begin{array}{c}\text { Inter- / intra- } \\
\text { species }\end{array}$ & $\begin{array}{l}\text { Control for } \\
\text { phylogeny }\end{array}$ \\
\hline Baker 2006 & $\begin{array}{l}\text { Western gerygones (Gerygone fusca } \\
\text { fusca), red-capped robins (Petroica } \\
\text { goodenovii) }\end{array}$ & intra-species & \\
\hline Brumm 2006 & nightingales (Luscinia megarhynchos) & intra-species & yes \\
\hline Dabelsteen \& Mathevon 2002 & blackcaps (Sylvia atricapilla) & intra-species & \\
\hline Dabelsteen et al. 1993 & black birds (Turdus merula) & intra-species & \\
\hline Hunter 1980 & great tits (Parus major) & intra-species & \\
\hline Jilka \& Leisler 1974 & $\begin{array}{l}\text { sedge warblers (Acrocephalus } \\
\text { schoenobaenus), great reed warblers }(A . \\
\text { arundinaceus), reed warblers }(A . \\
\text { scirpaceus })\end{array}$ & intra-species & \\
\hline Mathevon et al. 1996 & wrens (Troglodytes troglodytes) & intra-species & \\
\hline Mays et al. 2006 & steer's liocichla (Liocichla steerii) & intra-species & \\
\hline Nemeth et al. 2001 & $\begin{array}{l}\text { antbirds (Myrmothera campanisona, } \\
\text { Thamnophilus aethiops, Thamnophilus } \\
\text { amazonicus, Myrmotherula axillaris, } \\
\text { Herpsilochmus dorsimaculatus) }\end{array}$ & intra-species & yes \\
\hline $\begin{array}{l}\text { Slabbekoorn \& den Boer- } \\
\text { Visser } 2006\end{array}$ & great tits (Parus major) & intra-species & \\
\hline Slabbekoorn \& Peet 2003 & great tits (Parus major) & intra-species & \\
\hline
\end{tabular}


Appendix 1: continued

b.

\begin{tabular}{|c|c|c|c|}
\hline Study & Species & $\begin{array}{l}\text { Inter- / intra- } \\
\text { species }\end{array}$ & $\begin{array}{c}\text { Control for } \\
\text { phylogeny }\end{array}$ \\
\hline de la Torre \& Snowdon 2002 & pygmy marmosets (Cebuella pygmaea) & intra-species & \\
\hline Egnor et al. 2007 & cotton-top tamarins (Saguinus oedipus) & intra-species & yes \\
\hline Koda et al. 2008 & Japanese macaques (Macaca fuscata) & intra-species & \\
\hline Marler 1974 & arboreal primates & inter-species & \\
\hline Mitani et al. 1999 & $\begin{array}{l}\text { chimpanzees (Pan troglodytes } \\
\text { schweinfurthii) }\end{array}$ & intra-species & \\
\hline Parris 2002 & $\begin{array}{l}\text { Northern spring peepers (Pseudacris } c \text {. } \\
\text { crucifer) }\end{array}$ & intra-species & \\
\hline Rendall et al. 2000 & $\begin{array}{l}\text { chacma baboons (Papio cynocephalus } \\
\text { ursinus) }\end{array}$ & intra-species & yes \\
\hline Schneider et al. 2008 & $\begin{array}{l}\text { Siberut primates (Hylobates klossii, } \\
\text { Macaca siberu, Simias concolor, } \\
\text { Presbytis potenziani) }\end{array}$ & intra-species & \\
\hline Waser \& Brown 1986 & primates & inter-species & \\
\hline Waser \& Waser 1977 & forest monkeys & inter-species & \\
\hline Waser \& Waser 1977 & $\begin{array}{l}\text { black and white colobus (Colobus } \\
\text { guereza) }\end{array}$ & intra-species & \\
\hline Waser 1982 & $\begin{array}{l}\text { grey-cheeked mangabeys (Cercocebus } \\
\text { albigena), black mangabeys }(C . \\
\text { aterrimus), crested mangabeys }(C . \\
\text { galeritus), white-collared mangabeys } \\
\text { (C. torquatus), sooty mangabeys }(C . \\
\text { atys), baboons (Papio cynocephalus) }\end{array}$ & inter-species & \\
\hline $\begin{array}{l}\text { Waser, unpubl. in Waser \& } \\
\text { Waser } 1977\end{array}$ & $\begin{array}{l}\text { grey-cheeked mangabeys vs. baboons } \\
\text { (Cercocebus albigena vs. Papio } \\
\text { cynocephalus) }\end{array}$ & inter-species & \\
\hline
\end{tabular}


Appendix 2: Studies investigating the influences of the habitat on acoustic variables in birds. Studies used in the meta-analysis of Boncoraglio and Saino (2007) are in bold. Vocalisation type: "S": song, "C": call, "AdC": advertisement call. "yes": the habitat has an influence in accordance with a general prediction listed in Table 2.1. "no": no influence of the habitat was found. "inv": an influence of the habitat was found but the effect was contrary to that predicted (Table 2.1). The last column states whether study species were passerine birds ("yes"), non-passerine birds ("no") or a mix of both ("mix").

\begin{tabular}{|c|c|c|c|c|c|c|c|c|c|c|c|c|c|c|c|}
\hline 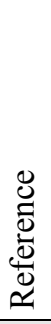 & Study & 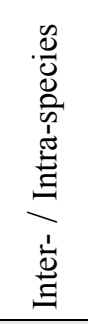 & Species name & 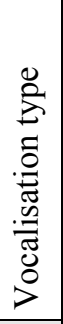 & 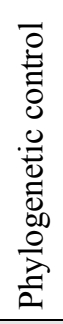 & 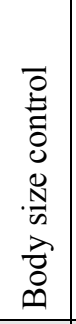 & 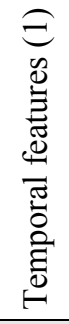 & 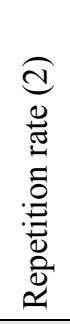 & 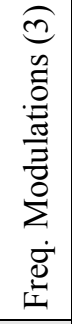 & 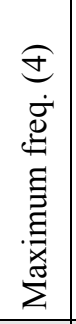 & 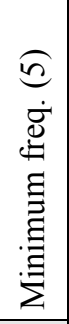 & 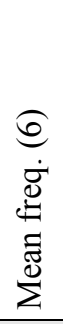 & 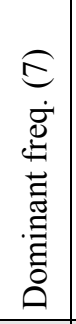 & 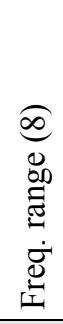 & 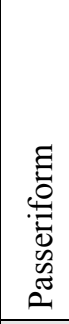 \\
\hline 1 & Anderson \& Conner 1985 & Intra & Cardinalis cardinalis & $\mathrm{S}$ & no & no & no & no & yes & & & & no & yes & yes \\
\hline 2 & Badyaev \& Leaf 1997 & Inter & Phylloscopus \& Hippolais warblers & $\mathrm{S}$ & no & yes & yes & yes & & inv & & & no & & yes \\
\hline 3 & Bergmann 1978 & Inter & 10 species of the Sylvia genus & $\mathrm{S}$ & no & no & yes & & & & & & & yes & yes \\
\hline 4 & Bertelli \& Tubaro 2002 & Inter & 39 tinamous species & $\mathrm{S}$ & yes & yes & & & & yes & yes & & yes & yes & no \\
\hline 5 & Blumstein \& Turner 2005 & Inter & 121 species of Australian songbirds & $\mathrm{S}$ & yes & yes & no & & & no & & & no & no & yes \\
\hline 6 & Bowman 1979 & Intra & Camarhynchus parvulus & $\mathrm{S}$ & no & no & & & & & & & yes & yes & yes \\
\hline 7 & Chapuis 1971 & Inter & 85 equatorial species & $\mathrm{S}$ & yes & yes & & yes & yes & & & yes & & yes & $\operatorname{mix}$ \\
\hline 8 & Cosens \& Falls 1984 & Inter & 11 bird species & $\mathrm{S}$ & no & yes & & & & no & yes & & yes & & $\operatorname{mix}$ \\
\hline 9 & Handford 1981 & Intra & Zonotrichia capensis & $\mathrm{S}$ & no & no & & no & & & & & & & yes \\
\hline 10 & Handford 1988 & Intra & Zonotrichia capensis & $\mathrm{S}$ & no & no & & no & & & & & & & yes \\
\hline 11 & Handford \& Lougheed 1991 & Intra & Zonotrichia capensis & $\mathrm{S}$ & no & yes & yes & & & inv & inv & & & inv & yes \\
\hline 12 & Hunter \& Krebs 1979 & Intra & Parus major & $\mathrm{S}$ & no & yes & & yes & & yes & no & & & yes & yes \\
\hline 13 & Hylton \& Godard 2001 & Intra & Passerina cyanea & $\mathrm{S}$ & no & no & no & no & inv & no & no & & no & & yes \\
\hline 14 & Jenkins \& Baker 1984 & Intra & Fringilla coelebs & $\mathrm{S}$ & no & no & & & yes & & & & & & yes \\
\hline 15 & Kopuchian et al. 2004 & Intra & Zonotrichia capensis & $\mathrm{S}$ & yes & no & no & yes & & no & yes & & yes & & yes \\
\hline 16 & Lemon et al. 1981 & Inter & American warblers & $\mathrm{S}$ & no & yes & no & & & yes & & & & no & yes \\
\hline 17 & Lijtmaer \& Tubaro 2007 & Intra & Zonotrichia capensis & $\mathrm{S}$ & yes & no & yes & inv & & yes & yes & & no & no & yes \\
\hline 18 & McCracken \& Sheldon 1997 & Inter & Herons: Ciconiiformes: Ardeidae & $\mathrm{C}$ & yes & no & & & & & & no & yes & yes & no \\
\hline 19 & Morton 1975 & Inter & 177 neotropical bird species & $\mathrm{S}$ & no & no & & & yes & & & & yes & yes & $\operatorname{mix}$ \\
\hline
\end{tabular}




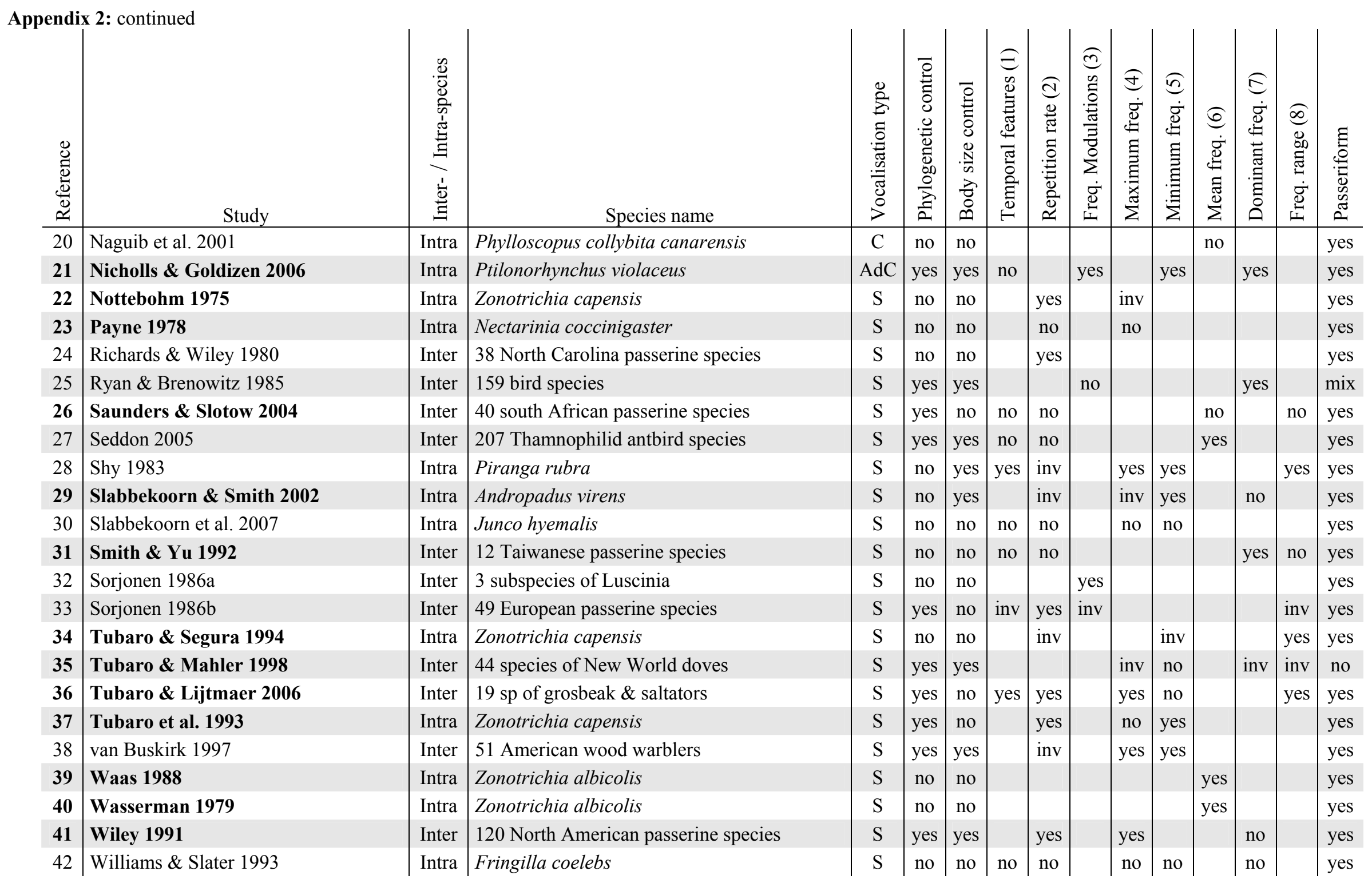


Appendix 3: Mean tree density and its standard error for each tree category (1: $5 \leq \mathrm{DBH}<10 \mathrm{~cm}, 2$ : $10 \leq \mathrm{DBH}<20 \mathrm{~cm}, 3: 20 \leq \mathrm{DBH}<30 \mathrm{~cm}, 4: \mathrm{DBH} \geq 30 \mathrm{~cm}$ ) in forest (triangles) and in woodland savannah (circles) in (a) Gamgam and (b) Kwano.

a.

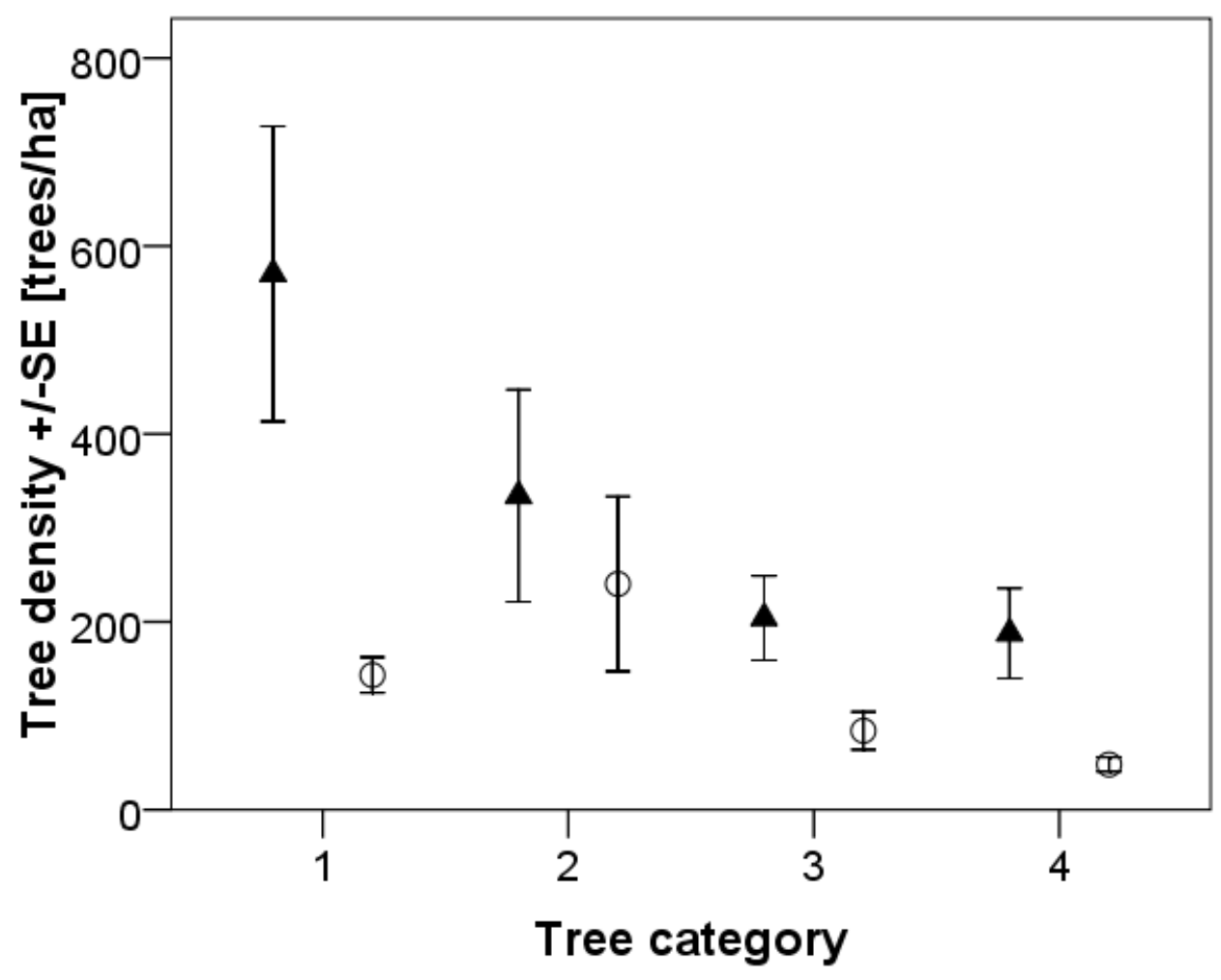

b.

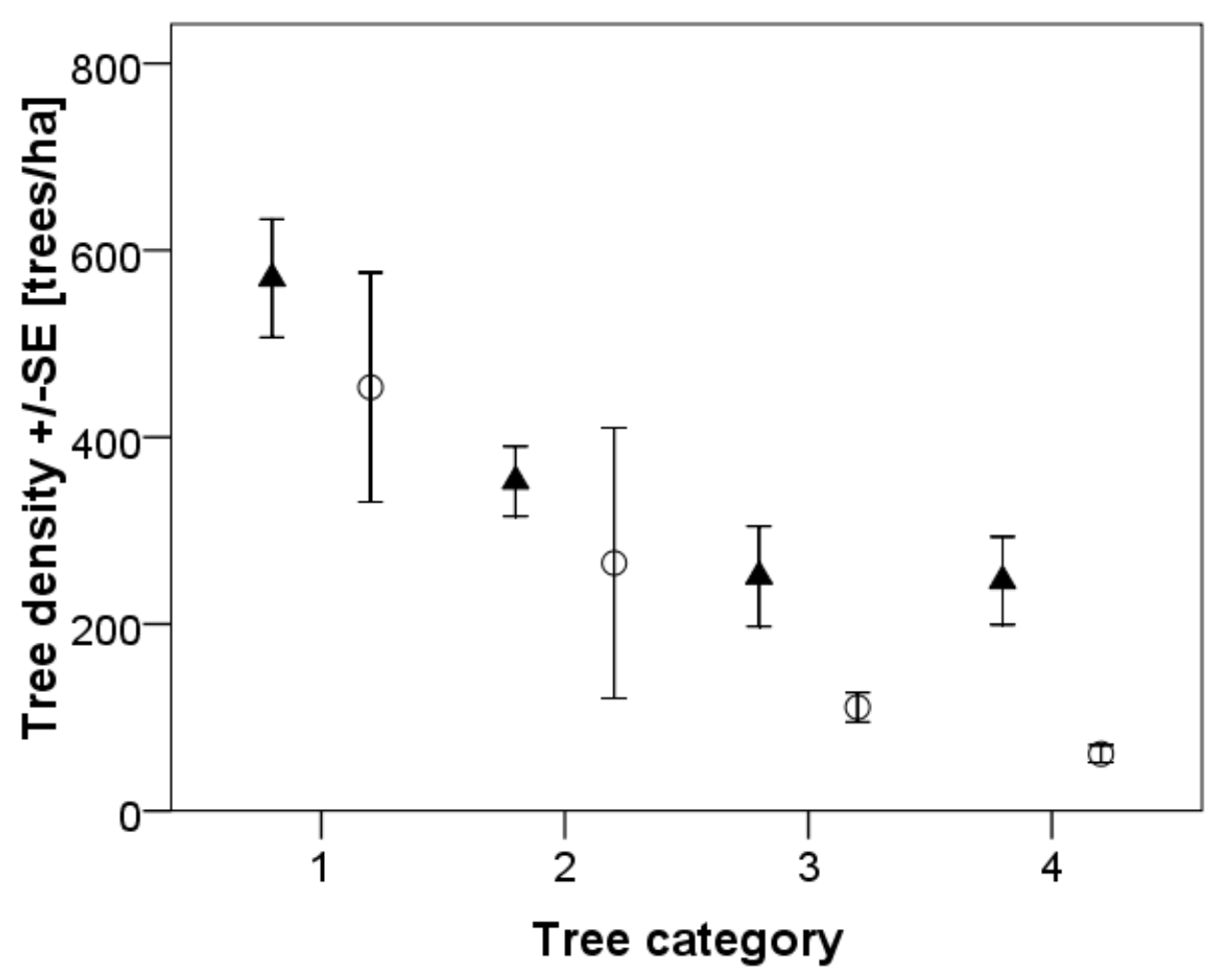


Appendix 4: Comparisons of visual vegetation densities between study sites in Nigeria (Gamgam and Kwano) and Uganda (Sonso; data collected by C. Rahn).

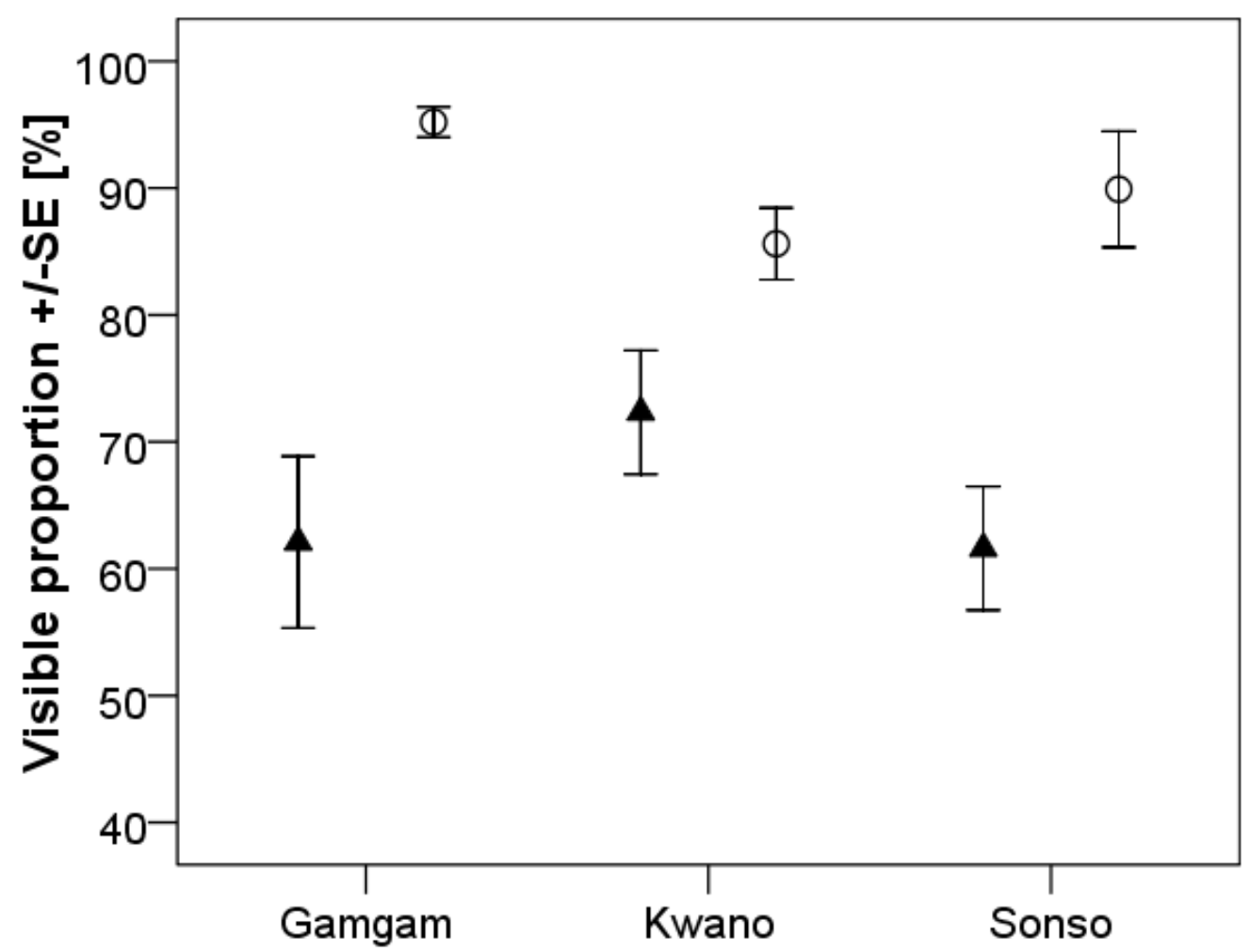

Figure: Mean visible proportion of a piece of tissue through vegetation (\%) and its standard error on pictures taken $1.5 \mathrm{~m}$ above ground from $5 \mathrm{~m}$ in forest (triangles) and in open habitat (circles).

Table: Results of exact 2-tailed Mann-Whitney U-tests used to compare visual vegetation densities (pictures taken $1.5 \mathrm{~m}$ above ground from $5 \mathrm{~m}$ ) between study sites in Nigeria (Gamgam and Kwano) and in Uganda (Sonso) within each habitat type.

\begin{tabular}{l|c|c} 
& Forest & Open habitat \\
\hline Uganda vs. Nigeria & $\begin{array}{l}\mathrm{U}=144, \mathrm{p}=0.309 \\
\left(\mathrm{~N}_{1}=15, \mathrm{~N}_{2}=24\right)\end{array}$ & $\begin{array}{c}\mathrm{U}=139, \mathrm{p}=0.143 \\
\left(\mathrm{~N}_{1}=13, \mathrm{~N}_{2}=15\right)\end{array}$ \\
& & \\
Gamgam vs. Kwano & $\mathrm{U}=52, \mathrm{p}=0.277$ & $\mathrm{U}=45, \mathrm{p}=0.004$ \\
& $\left(\mathrm{~N}_{1}=11, \mathrm{~N}_{2}=13\right)$ & $\left(\mathrm{N}_{1}=15, \mathrm{~N}_{2}=15\right)$ \\
Gamgam vs. Sonso & $\mathrm{U}=80, \mathrm{p}=0.919$ & $\mathrm{U}=83, \mathrm{p}=0.525$ \\
& $\left(\mathrm{~N}_{1}=11, \mathrm{~N}_{2}=15\right)$ & $\left(\mathrm{N}_{1}=15, \mathrm{~N}_{2}=13\right)$ \\
Kwano vs. Sonso & $\mathrm{U}=64, \mathrm{p}=0.130$ & $\mathrm{U}=56, \mathrm{p}=0.058$ \\
& $\left(\mathrm{~N}_{1}=13, \mathrm{~N}_{2}=15\right)$ & $\left(\mathrm{N}_{1}=15, \mathrm{~N}_{2}=13\right)$
\end{tabular}




\section{Curriculum vitae}

\section{Elodie Ey}

Date of birth: March $23^{\text {rd }} 1982$

Place of birth: Mulhouse (France)

Nationality: French

Single

\section{EDUCATION}

since 2005 German Primate Centre, Göttingen.

$\mathrm{PhD}$ study: "Influences of ecological factors on vocal communication in olive baboons (Papio hamadryas anubis)". Tutor: Prof. Dr. J. Fischer.

2002-2005 Institut National Agronomique Paris-Grignon, post-graduate engineering school for agricultural and life sciences.

Master degree "Sciences and Techniques of Living being", option "Animal Sciences" with orientation "Behavioural Sciences".

2000-2002 Lycée Jean Rostand, Strasbourg.

Preparatory class for admission to post-graduate engineering top schools, specializing in biology, mathematics, physics, and chemistry.

$2000 \quad$ Lycée Jean-Henri Lambert, Mulhouse.

High school diploma (Baccalauréat), majored in mathematics.

\section{RELEVANT EXPERIENCE}

2005-2008 German Primate Centre, Göttingen.

PhD study: 10-month data collection in two troops of olive baboons in Gashaka-Gumti National Park, Nigeria. Acoustic and statistical analyses of data at the German Primate Centre, Göttingen.

Feb-Jun 2005 German Primate Centre, Göttingen.

Master thesis: "Age- and sex-related variations in clear calls of chacma baboon (Papio cynocephalus ursinus)". Tutor: Prof. Dr. Julia Fischer.

Jun-Aug 2004 Institute of Zoology, School of Veterinary Medicine, Hanover.

Training period: "Social signals of chimpanzees (Pan troglodytes), focusing on laughter and smile". Tutor: Prof. Dr. Elke Zimmermann.

March 2004 Laboratory of Ecology, Systematic and Evolution, Orsay.

Study on the relationship between sexual dimorphism and longevity in birds. Tutor: Dr. Franck Courchamp and Dr. Jacqui Shykoff

July 2003 Zoological park, Mulhouse.

Training period as health assistant, focusing on the adaptation of cares to wild behaviour of captive animals.

Apr-May 2003 Dairy farm, Calvados (France)

Discovering the agricultural sphere and studying the running of a family firm. 


\section{PUBLICATIONS}

Ey E., Fischer J. In press. Keeping in contact - Flexibility in calls of olive baboons. In Sommer V. and Ross C. (Eds), Primates of Gashaka. Socio-ecology and Conservation in Nigeria's Biodiversity Hotspot. Series: Developments in Primatology: Progress and Prospects. Springer, New York.

Ey E., Hammerschmidt K., Fischer J. 2007. Age- and sex-related variations in clear calls of Chacma baboons (Papio cynocephalus ursinus). International Journal of Primatology 28: 947-960.

Ey E., Pfefferle D., Fischer J. 2007. Age- and sex-related variations as cues for body size in sound production - a review in non-human primates. Primates 48: 253-267. 